Supporting Information

\title{
Synthesis of Sulfones via Ru(II)-Catalyzed Sulfination of Boronic Acids
}

\author{
Krista Gulbe and Māris Turks* \\ Institute of Technology of Organic Chemistry, Faculty of Materials Science and Applied Chemistry, \\ Riga Technical University, P. Valdena Street 3, Riga, LV-1048, Latvia \\ *Corresponding Author \\ E-mail: Maris.Turks@rtu.lv
}

\section{Contents:}

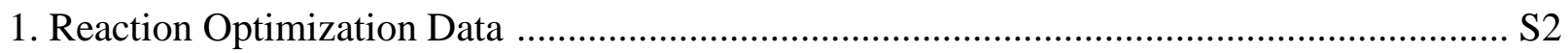

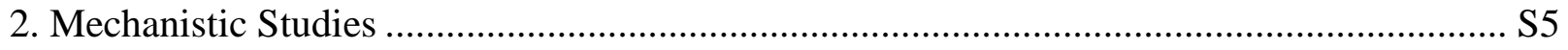

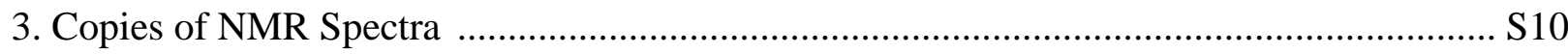




\section{Reaction Optimization Data}

Table S1. Screening of the Base Additive ${ }^{a}$

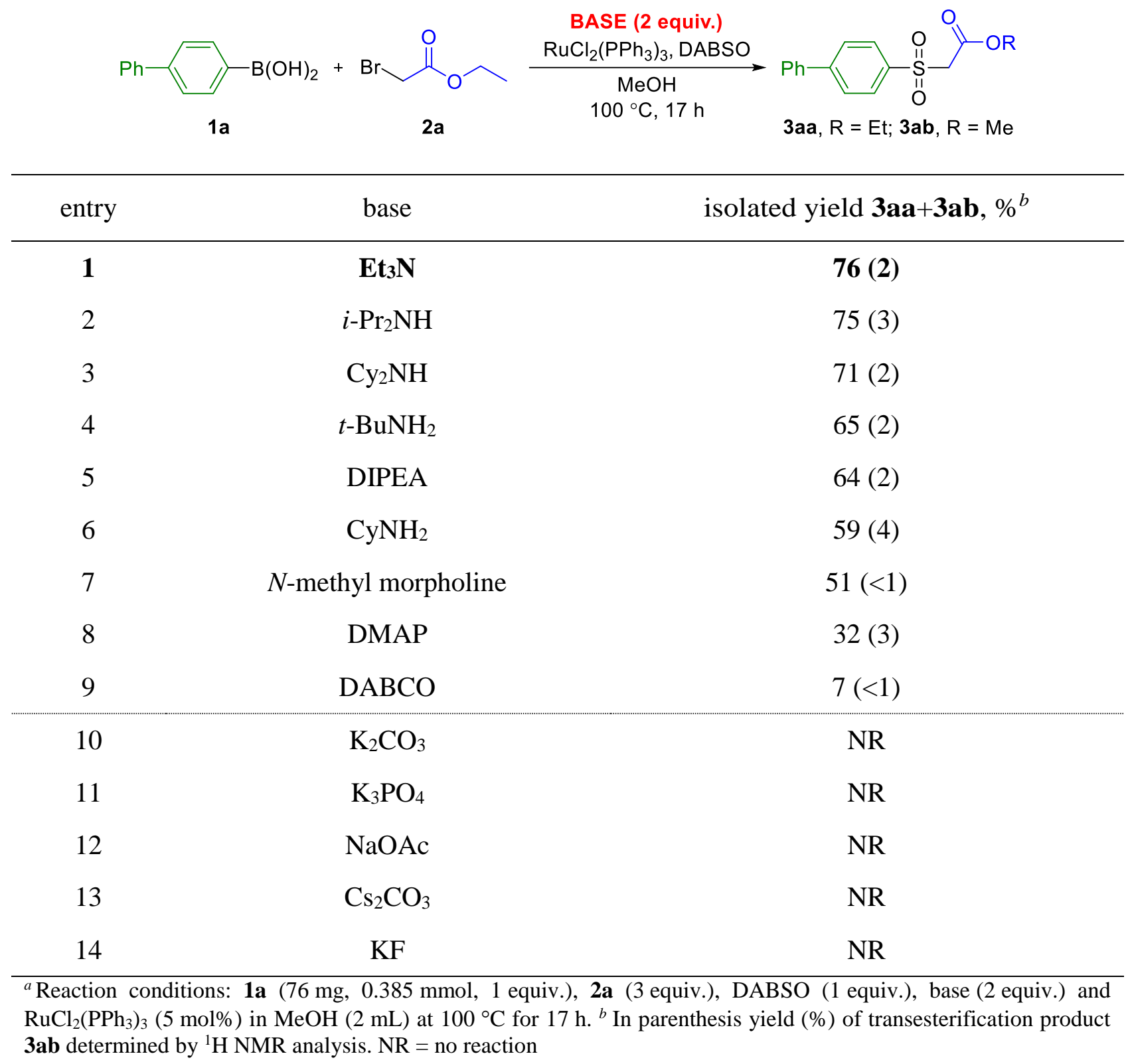


Table S2. Solvent Screening ${ }^{a}$

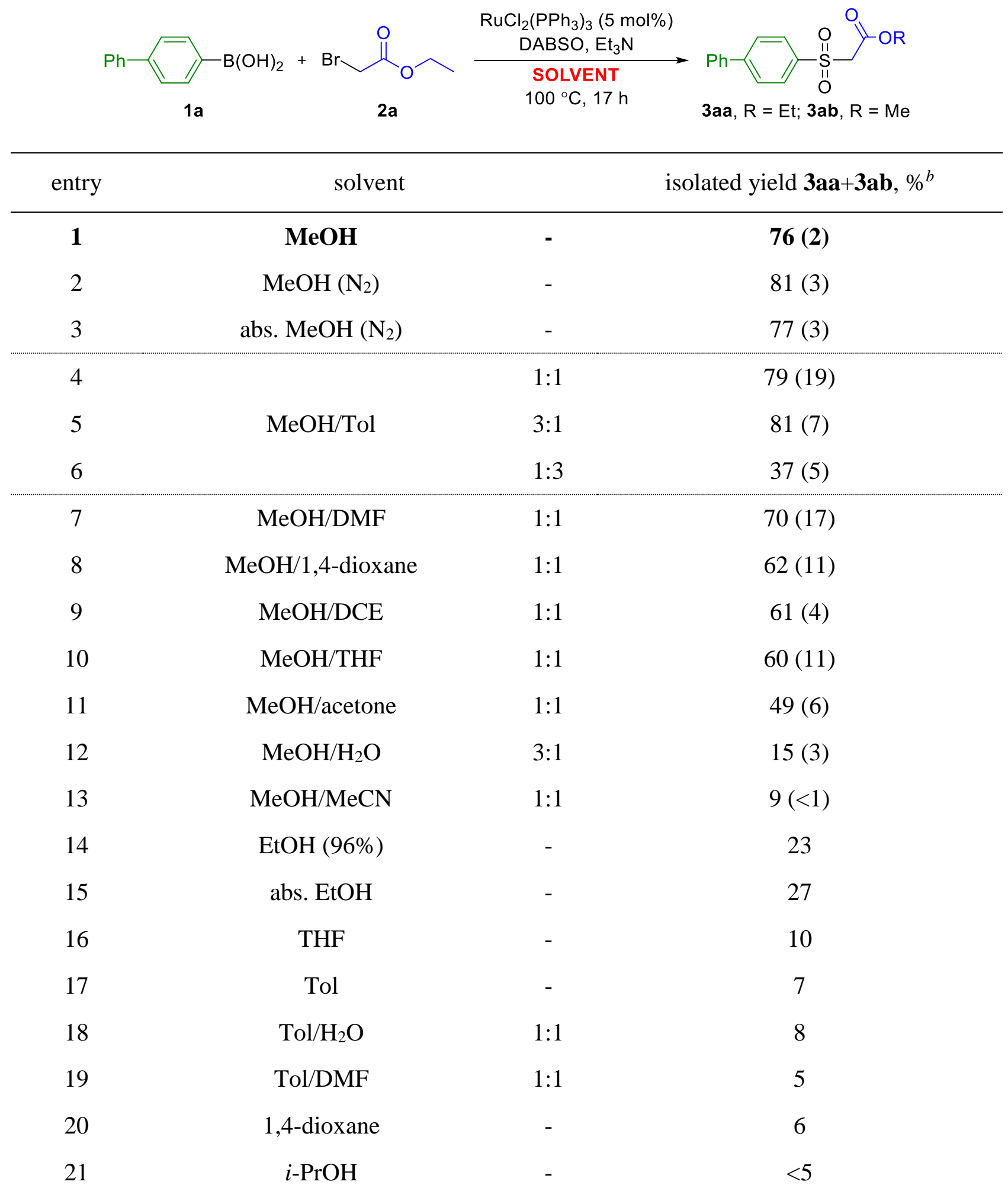

${ }^{a}$ Reaction conditions: 1a $\left(76 \mathrm{mg}, 0.385 \mathrm{mmol}, 1\right.$ equiv.), 2a (3 equiv.), DABSO (1 equiv.), $\mathrm{Et}_{3} \mathrm{~N}$ ( 2 equiv.) and $\mathrm{RuCl}_{2}\left(\mathrm{PPh}_{3}\right)_{3}(5 \mathrm{~mol} \%)$ in solvent $(2 \mathrm{~mL})$ at $100{ }^{\circ} \mathrm{C}$ for $17 \mathrm{~h} .{ }^{b}$ In parenthesis yield (\%) of transesterification product 3ab determined by NMR analysis. 
Table S3. Final Optimization of the Reaction Conditions ${ }^{a}$

\begin{tabular}{|c|c|c|c|}
\hline & $1 a$ & 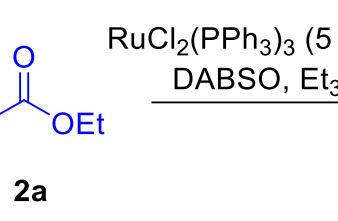 & 3aa, $\mathrm{R}=\mathrm{Et} ; 3 \mathbf{3 a b}, \mathrm{R}=\mathrm{Me}$ \\
\hline entry & solvent & variable & isolated yield $\mathbf{3 a a}+\mathbf{3 a b}, \%^{b}$ \\
\hline 1 & \multirow{4}{*}{$\begin{array}{c}\mathrm{MeOH} / \mathrm{Tol} \\
1: 1\end{array}$} & - & $79(19)$ \\
\hline 2 & & 10 mol $\%$ cat. & $80(18)$ \\
\hline 3 & & $1 \mathrm{~mol} \%$ cat. & $54(14)$ \\
\hline 4 & & at $65^{\circ} \mathrm{C}$ & $13(-)$ \\
\hline 5 & \multirow{9}{*}{$\mathrm{MeOH}$} & - & $76(2)$ \\
\hline 6 & & 2 equiv. $2 \mathbf{a}$ & $39(2)$ \\
\hline 7 & & 3 equiv. $\mathrm{Et}_{3} \mathrm{~N}$ & $71(15)$ \\
\hline 8 & & $30 \mathrm{~mol} \%$ AgOTf & $67(<1)$ \\
\hline 9 & & $30 \mathrm{~mol} \% \mathrm{TBAB}$ & $73(3)$ \\
\hline 10 & & $8 \mathrm{~h}$ & $69(2)$ \\
\hline 11 & & at $80^{\circ} \mathrm{C}$ & $66(<1)$ \\
\hline $12^{c}$ & & 0.6 equiv. DABSO & 3ab, 53 \\
\hline $13^{c}$ & & $\mathrm{DMAP} \cdot \mathrm{SO}_{2}$ & 3ab, 51 \\
\hline
\end{tabular}

${ }^{a}$ Initial reaction conditions: 1a $\left(0.385\right.$ mmol, 1 equiv.), 2a (3 equiv.), DABSO (1 equiv.), $\mathrm{Et}_{3} \mathrm{~N}$ (2 equiv.) and $\mathrm{RuCl}_{2}\left(\mathrm{PPh}_{3}\right)_{3}(5 \mathrm{~mol} \%)$ in a solvent $(2 \mathrm{~mL})$ at $100{ }^{\circ} \mathrm{C}$ for $17 \mathrm{~h} .{ }^{b}$ In parenthesis yield $(\%)$ of transesterification product 3ab determined by NMR analysis. ${ }^{c}$ Methyl bromoacetate (2b) used as an electrophile. 


\section{Mechanistic Studies}

\section{A. Detection of sulfinate intermediate 6 (Figure S1 and Figure S2)}

Formation of sulfinate intermediate 6 was proved by NMR spectroscopy, particularly, by ${ }^{13} \mathrm{C}$ NMR experiment (Figure S1). Spectra of reaction mixtures were compared with the standards of boronic acid $\mathbf{1 h}$, sulfinate $\mathbf{6}(\mathrm{Y}=\mathrm{Na})$ and $\mathrm{PPh}_{3}$ (free and coordinated). The standard reaction was performed in MeOD- $d_{4}$ and analyzed without additional treatment. When the experiment was performed in the absence of $\mathrm{Et}_{3} \mathrm{~N}$, the reaction mixture was turbid. Therefore, it was filtered through a celite layer prior to NMR analysis.

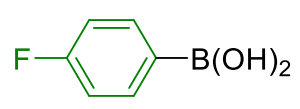

$1 \mathrm{~h}$

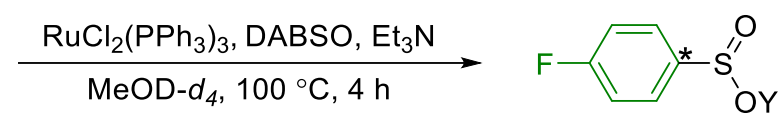

6

Sample preparation. 4-Fluorophenylboronic acid (1h, $28 \mathrm{mg}, 0.2 \mathrm{mmol}, 1$ equiv.), DABSO (1 equiv.) and $\mathrm{RuCl}_{2}\left(\mathrm{PPh}_{3}\right)_{3}(5 \mathrm{~mol} \%)$ were placed into a glass pressure tube $(5 \mathrm{~mL})$ equipped with a magnetic stirring bar. Then MeOD- $d_{4}(1 \mathrm{~mL})$ was added followed by $\mathrm{Et}_{3} \mathrm{~N}$ ( 2 equiv.). The resulting mixture was stirred at $100{ }^{\circ} \mathrm{C}$ (oil bath) for $4 \mathrm{~h}$. After cooling to room temperature, the reaction mixture was analyzed using NMR spectroscopy.

Conclusions: In the presence of Et ${ }_{3} N$, full consumption of boronic acid $\mathbf{1 h}$ and formation of sulfinate salt 6 were observed (Figure S1, III). Without the amine only partial conversion of boronic acid $\mathbf{1 h}$ was detected (Figure S1, V). Discrepancy of chemical shifts for the sulfur bonded carbon (*) in the sulfinate salt 6 may point to the different nature of counterion Y. Formation of corresponding mono- and dimethyl esters of aryl boronic acid under reaction conditions could lead to the changes in multiplicities detected in spectra $\boldsymbol{V}$ (Figure S1) for the residue of boronic acid $\mathbf{1 h}$.

\section{B. Detection of $\mathbf{B}(\mathbf{O M e}) \mathbf{3}$ (Figure S3 and Figure S4)}

To provide complete proposal for the reaction mechanism, possible formation of $\mathrm{B}(\mathrm{OMe})_{3}$ was investigated. Firstly, formation of $\mathrm{B}(\mathrm{OMe})_{3}$ was detected with $\mathrm{GC}-\mathrm{FID}$ by comparing chromatograms of reaction mixture and commercially available $\mathrm{B}(\mathrm{OMe})_{3}$ sample in $\mathrm{MeOH}$. Secondly, ${ }^{11} \mathrm{~B} \mathrm{NMR}$ spectrum of the reaction mixture confirmed full conversion of boronic acid into trimethyl borate.

Sample preparation. 4-Fluorophenylboronic acid (1h, $54 \mathrm{mg}, \quad 0.385 \mathrm{mmol}, \quad 1$ equiv.), DABSO (1 equiv.) and $\mathrm{RuCl}_{2}\left(\mathrm{PPh}_{3}\right)_{3}(5 \mathrm{~mol} \%)$ were placed into a glass pressure tube $(15 \mathrm{~mL})$ equipped with a magnetic stirring bar. Then $\mathrm{MeOH}(2 \mathrm{~mL})$ was added followed by $\mathrm{Et}_{3} \mathrm{~N}$ ( 2 equiv.) and methyl 2-bromoacetate (2a, 3 equiv.). The resulting mixture was stirred at $100{ }^{\circ} \mathrm{C}$ (oil bath) for $8 \mathrm{~h}$. After cooling to room temperature, the reaction mixture was centrifuged and the clear supernatant was used for GC-FID analysis and ${ }^{11} \mathrm{~B}$ NMR spectroscopic studies. 


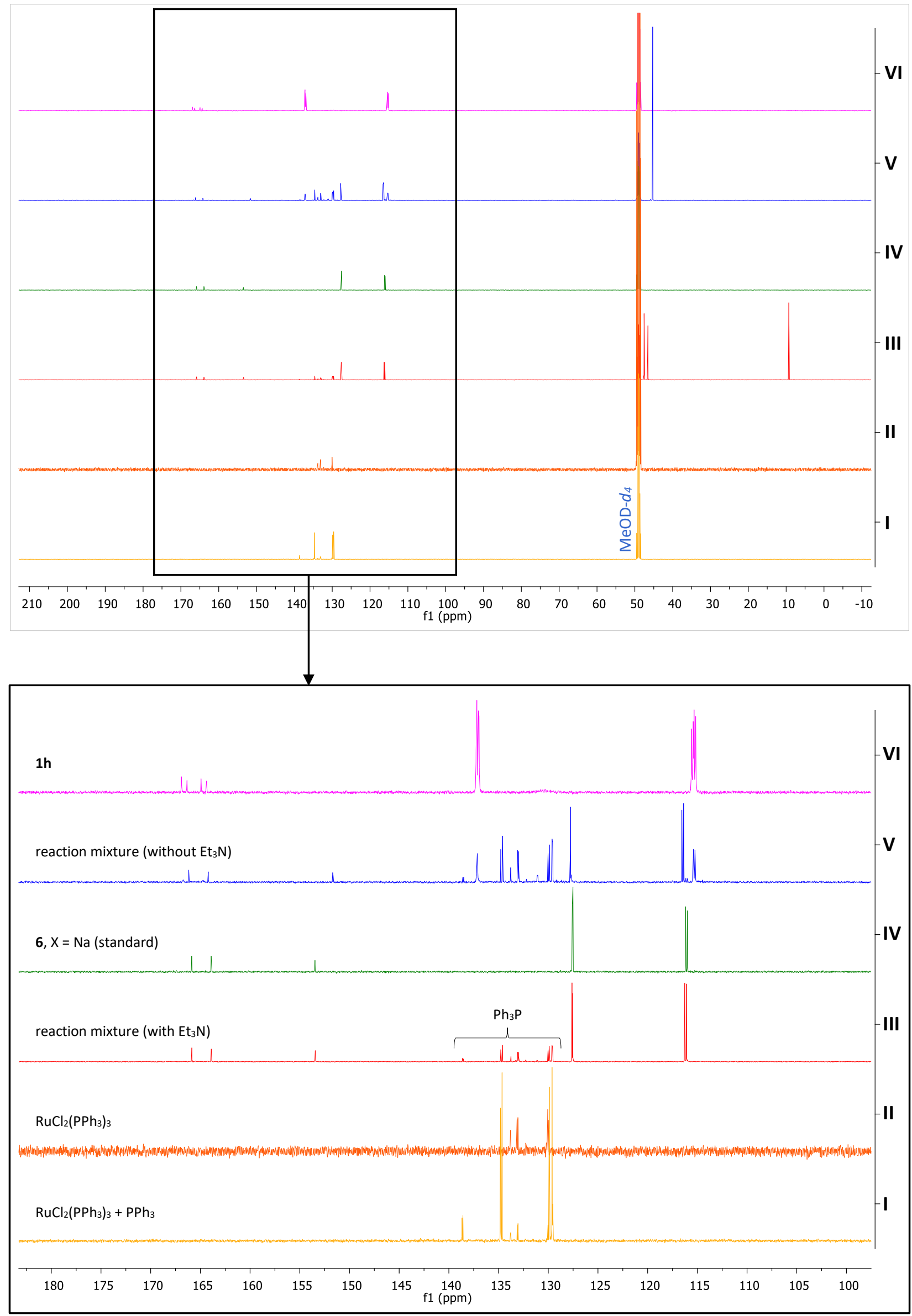

Figure S1. Detection of Sulfinate Intermediate 6: ${ }^{13} \mathrm{C}\left\{{ }^{1} \mathrm{H}\right\}$ NMR $\left(126 \mathrm{MHz}, \mathrm{MeOD}-d_{4}\right)$ spectra 


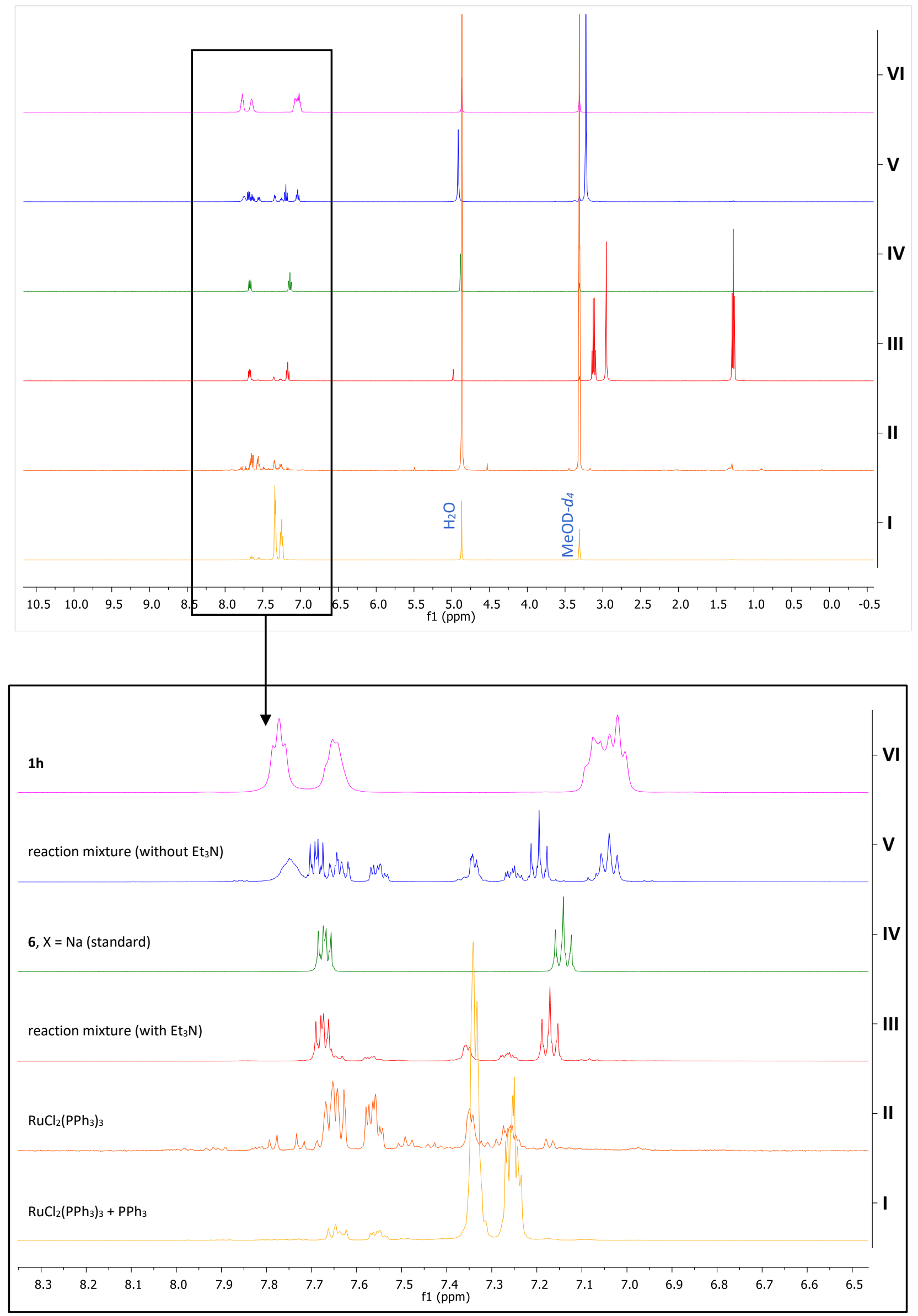

Figure S2. Detection of Sulfinate Intermediate 6: ${ }^{1} \mathrm{H}$ NMR $\left(500 \mathrm{MHz}, \mathrm{MeOD}-d_{4}\right)$ spectra 


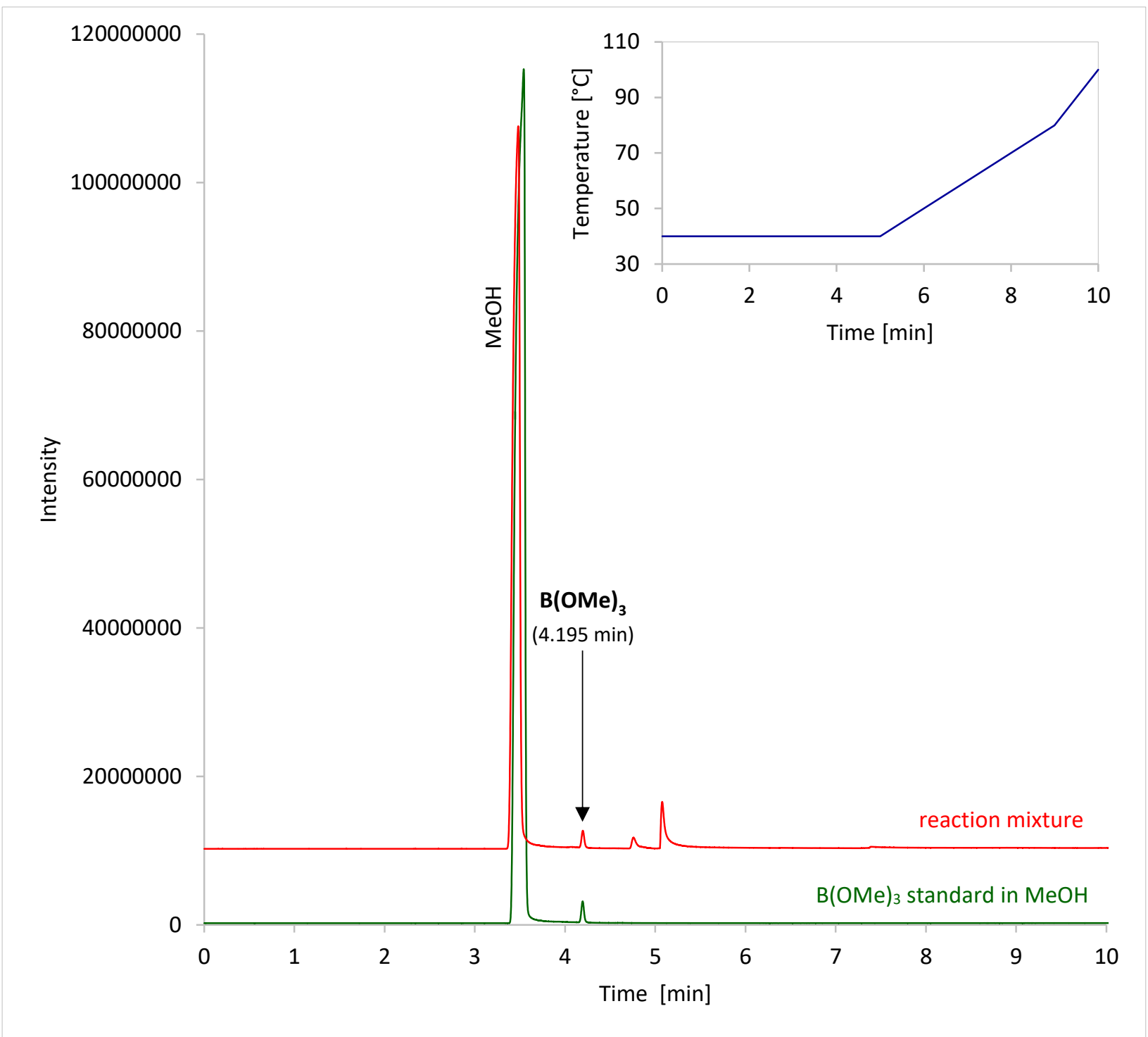

Figure S3. Detection of $\mathrm{B}(\mathrm{OMe})_{3}$ : GC-FID analysis

Table S4. Parameters for GC-FID Analysis (Figure S3)

\begin{tabular}{|ll|}
\hline Column & $\begin{array}{l}\text { Agilent Technologies } J \& W D B-1 m s \text { capillary column, } \\
30 \mathrm{~m} \times 0.32 \mathrm{mmID}, 0.25 \mu \mathrm{m}\end{array}$ \\
\hline Column temperature & $\begin{array}{l}\left.40^{\circ} \mathrm{C} \text { (hold for } 5 \mathrm{~min}\right) \text { to } 80^{\circ} \mathrm{C} @ 10^{\circ} \mathrm{C} / \mathrm{min} \text { to } 100^{\circ} \mathrm{C} @ \\
20^{\circ} \mathrm{C} / \mathrm{min} \text { (hold for } 1 \mathrm{~min} \text { at } 100^{\circ} \mathrm{C} \text { ) }\end{array}$ \\
\hline Injector/Detector (FID) & $250^{\circ} \mathrm{C} / 250^{\circ} \mathrm{C}$ \\
\hline Carrier gas & $\mathrm{He} @ 2 \mathrm{ml} / \mathrm{min}$, constant flow \\
\hline Injection regime & Splitless, no solvent delay \\
\hline Injection volume & $2 \mu \mathrm{L}$ \\
\hline
\end{tabular}




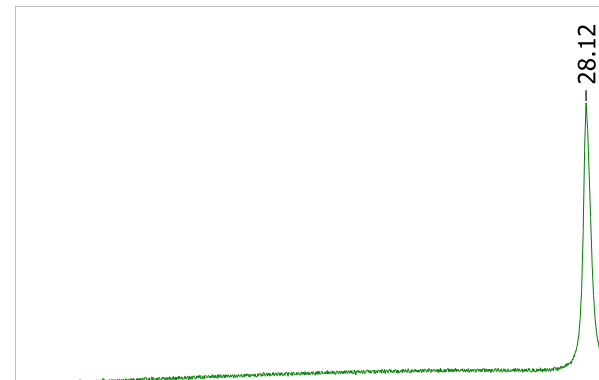

1h (standard)

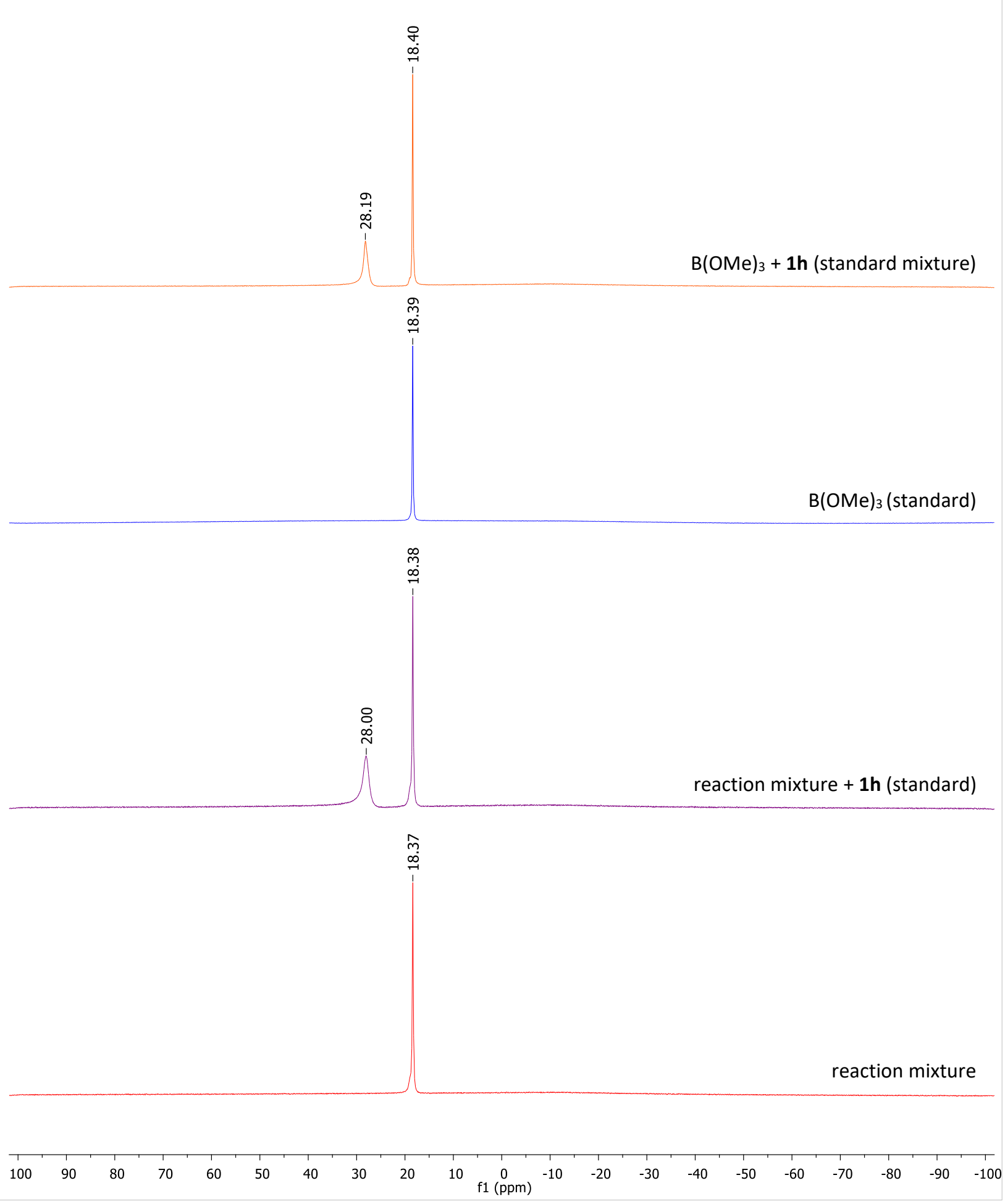

Figure S4. Detection of $\mathrm{B}(\mathrm{OMe})_{3}:{ }^{11} \mathrm{~B}$ NMR $\left(160 \mathrm{MHz}, \mathrm{MeOH}+10\right.$ vol\% DMSO- $\left.d_{6}\right)$ spectra 
3. Copies of NMR Spectra 
${ }^{1} \mathrm{H}$ NMR (500 MHz, $\mathrm{CDCl}_{3}$ )

3aa

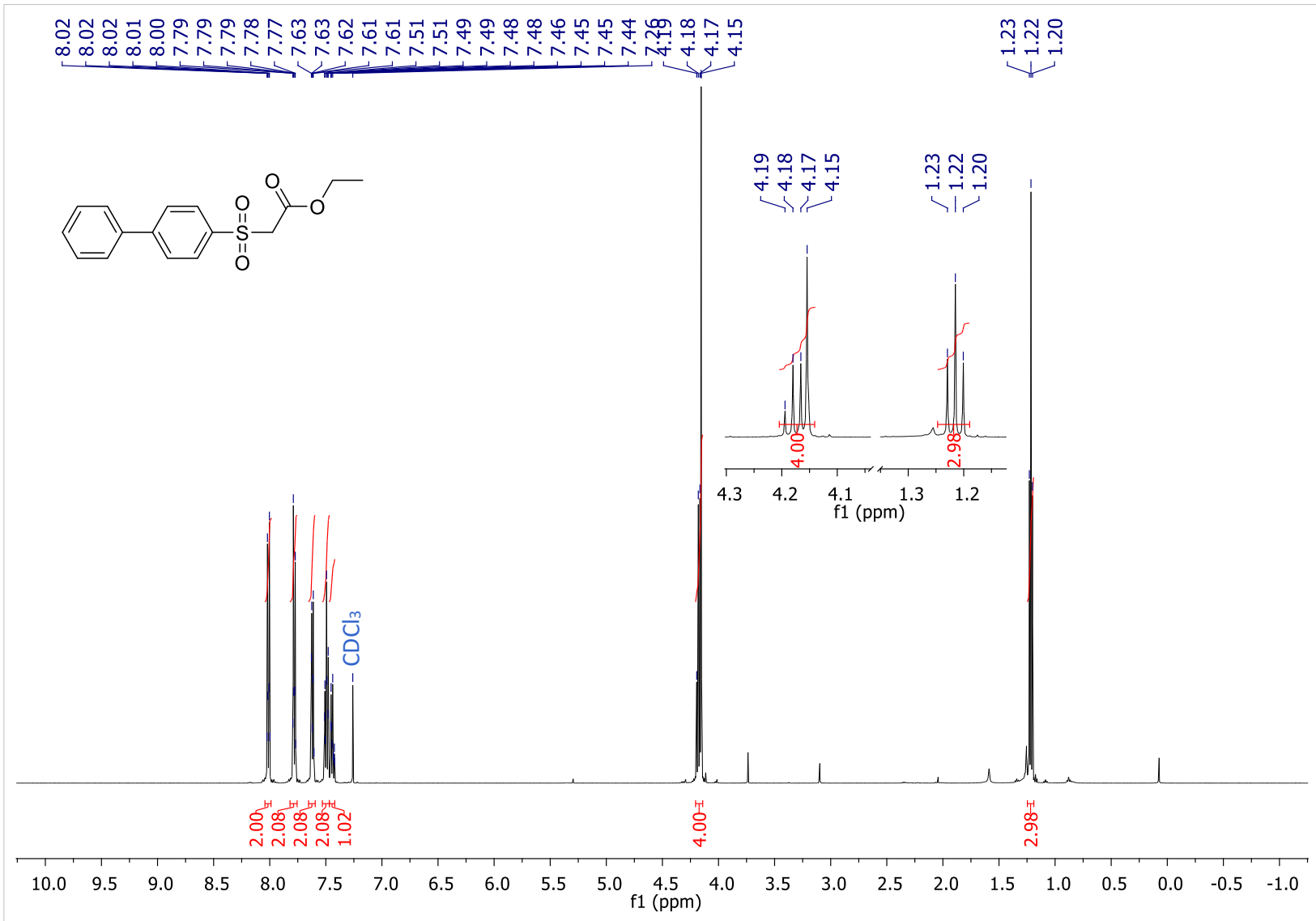

${ }^{13} \mathrm{C}\left\{{ }^{1} \mathrm{H}\right\}$ NMR $\left(126 \mathrm{MHz}, \mathrm{CDCl}_{3}\right)$

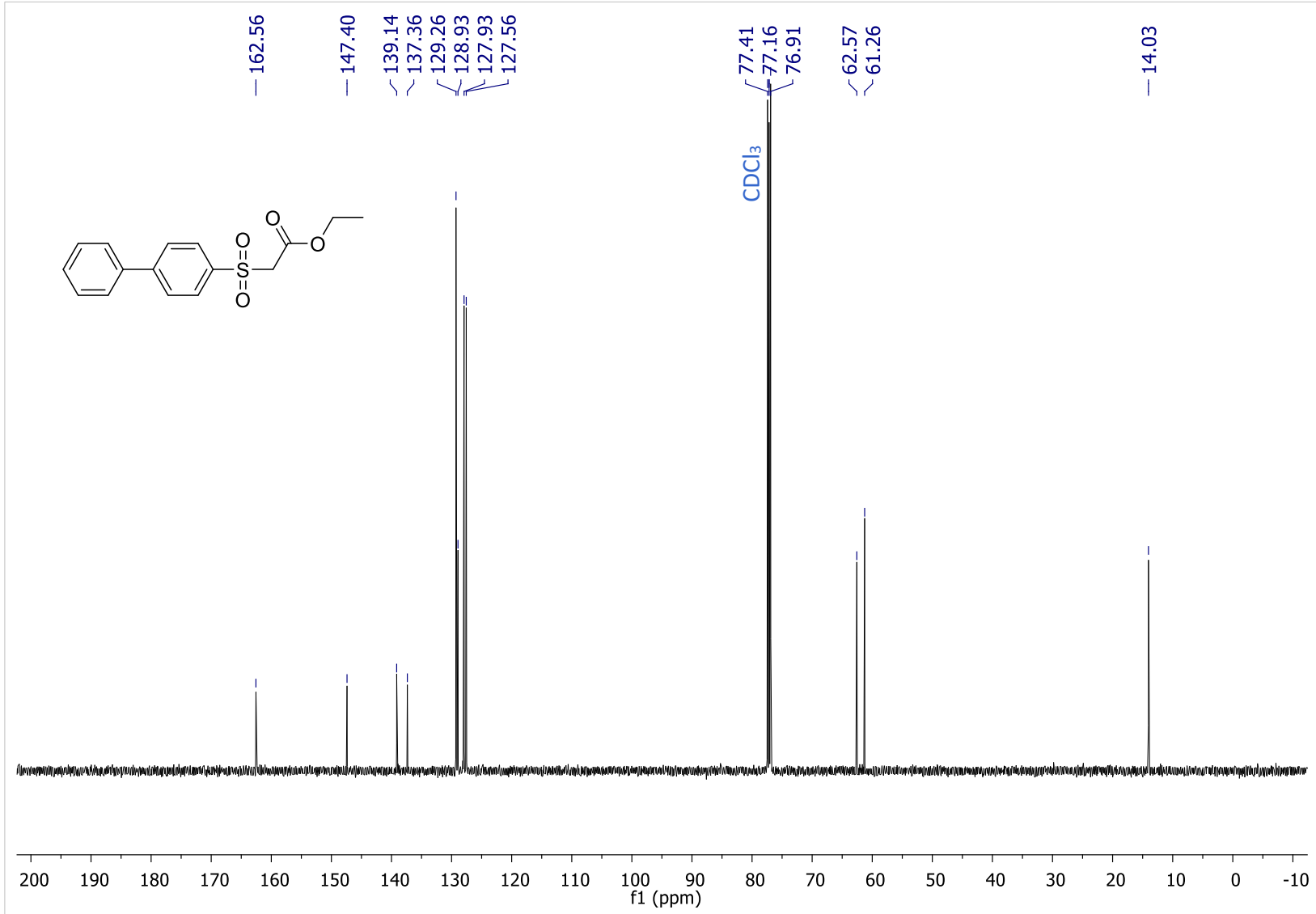




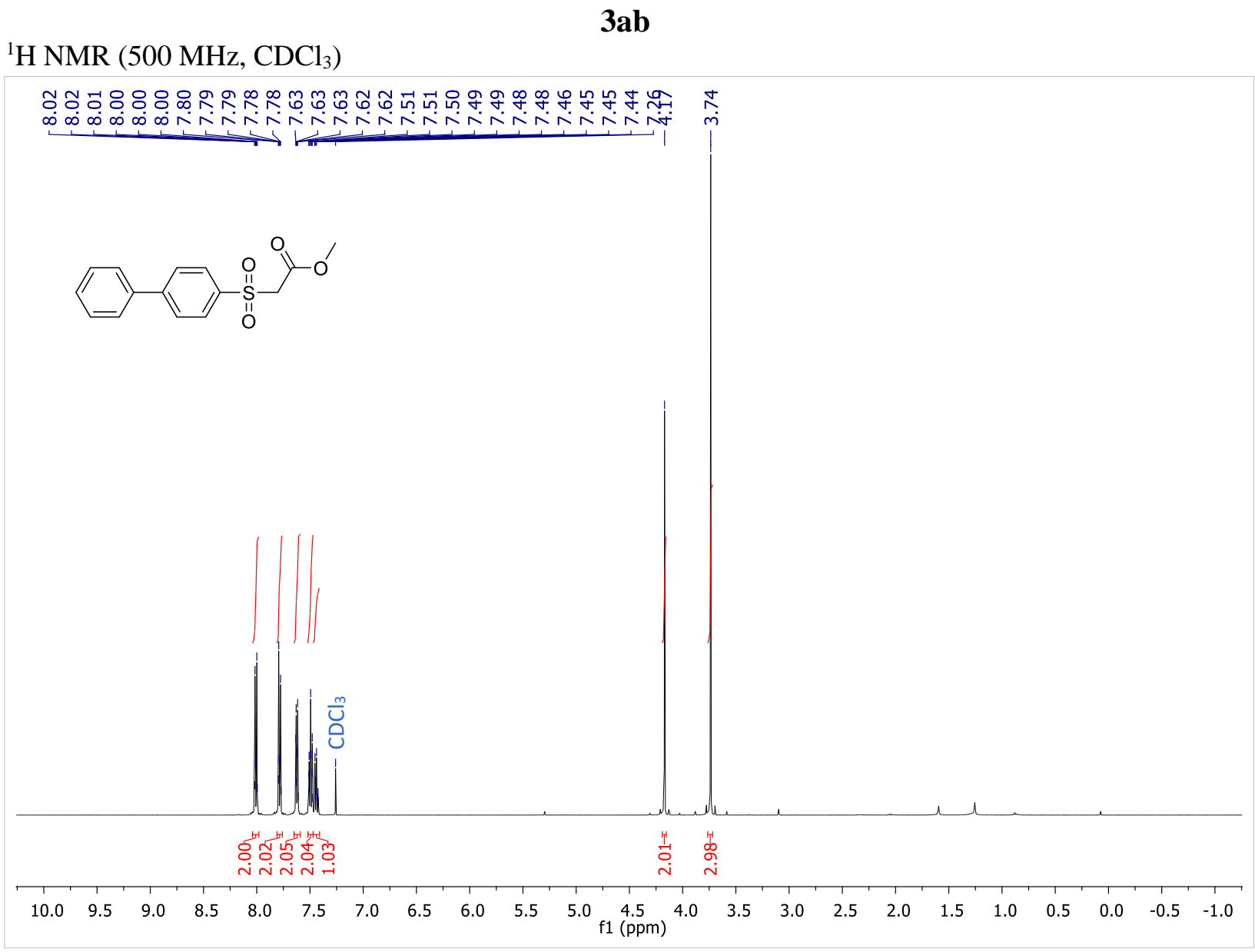

${ }^{13} \mathrm{C}\left\{{ }^{1} \mathrm{H}\right\} \mathrm{NMR}\left(126 \mathrm{MHz}, \mathrm{CDCl}_{3}\right)$

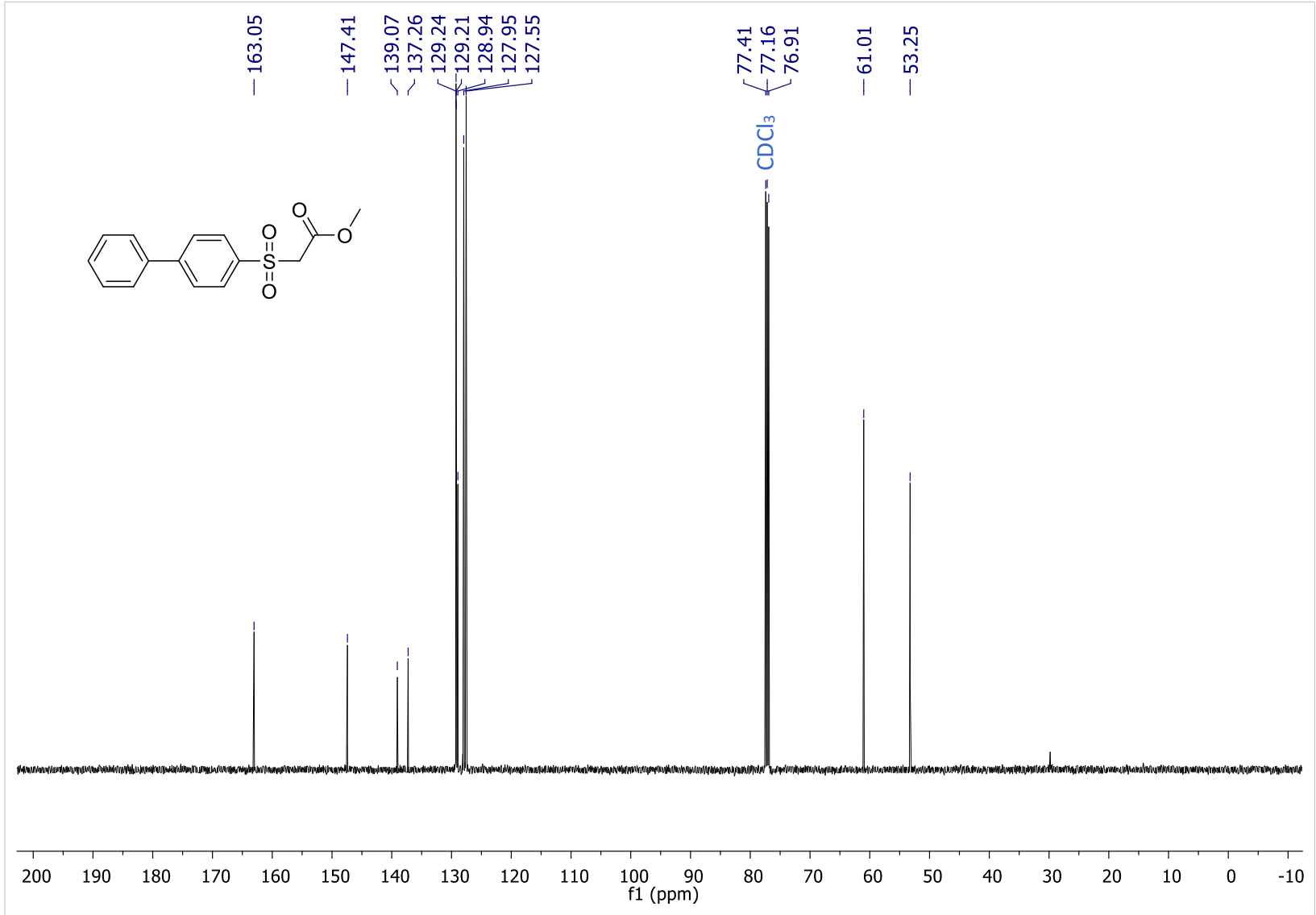


3bb

${ }^{1} \mathrm{H} \mathrm{NMR}\left(500 \mathrm{MHz}, \mathrm{CDCl}_{3}\right)$

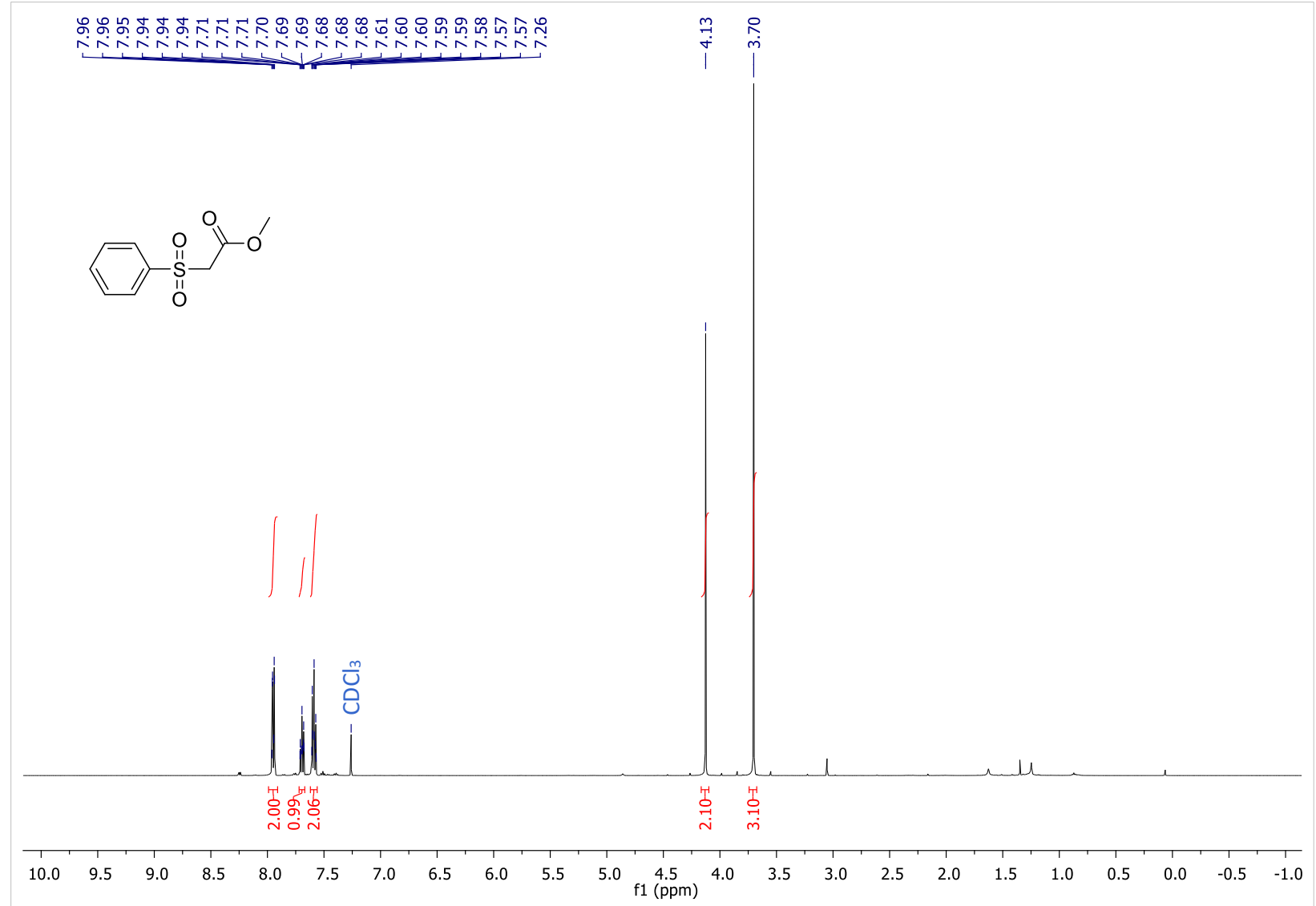

${ }^{13} \mathrm{C}\left\{{ }^{1} \mathrm{H}\right\} \mathrm{NMR}\left(126 \mathrm{MHz}, \mathrm{CDCl}_{3}\right)$

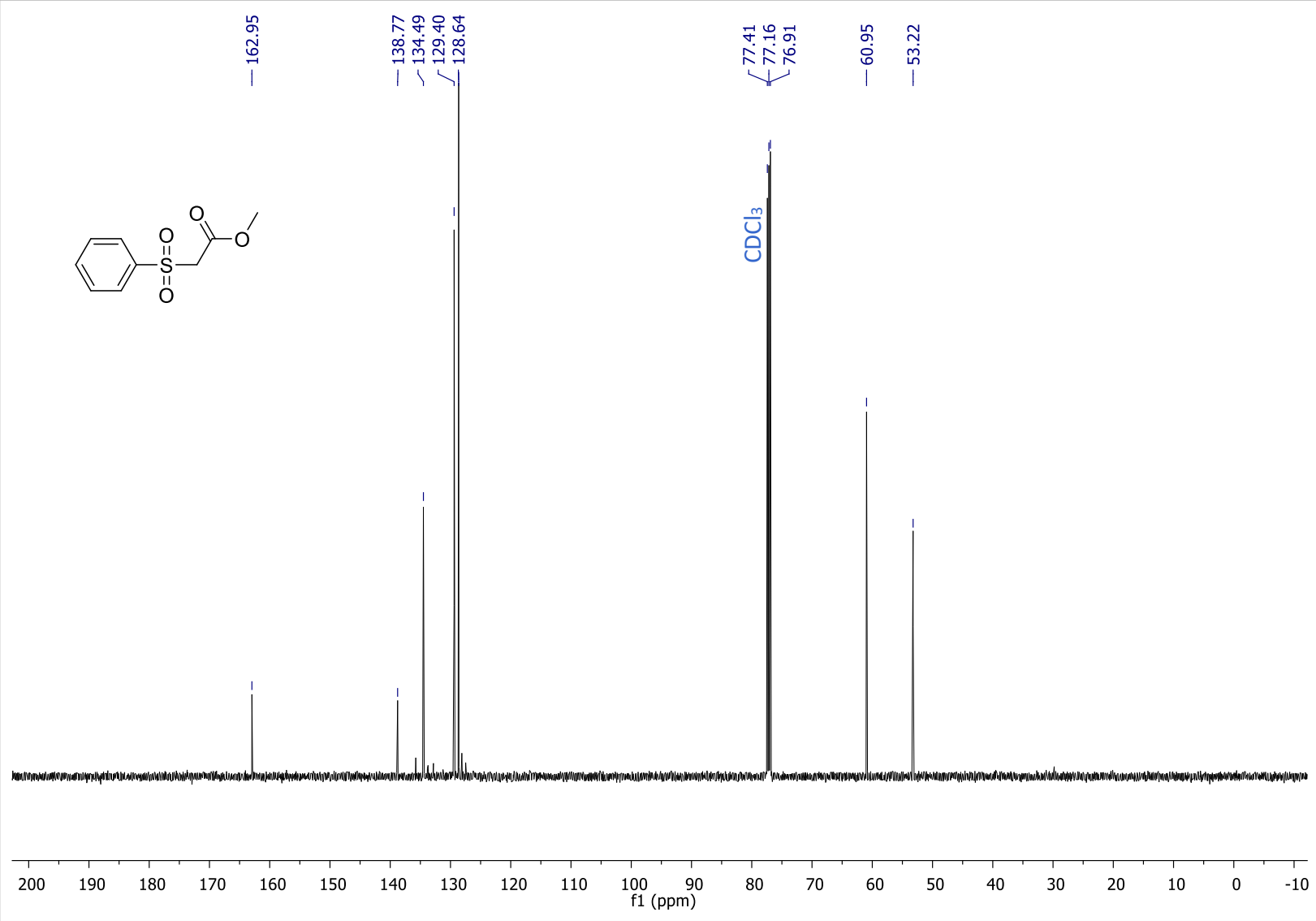


$3 c b$

${ }^{1} \mathrm{H}$ NMR (500 MHz, $\mathrm{CDCl}_{3}$ )

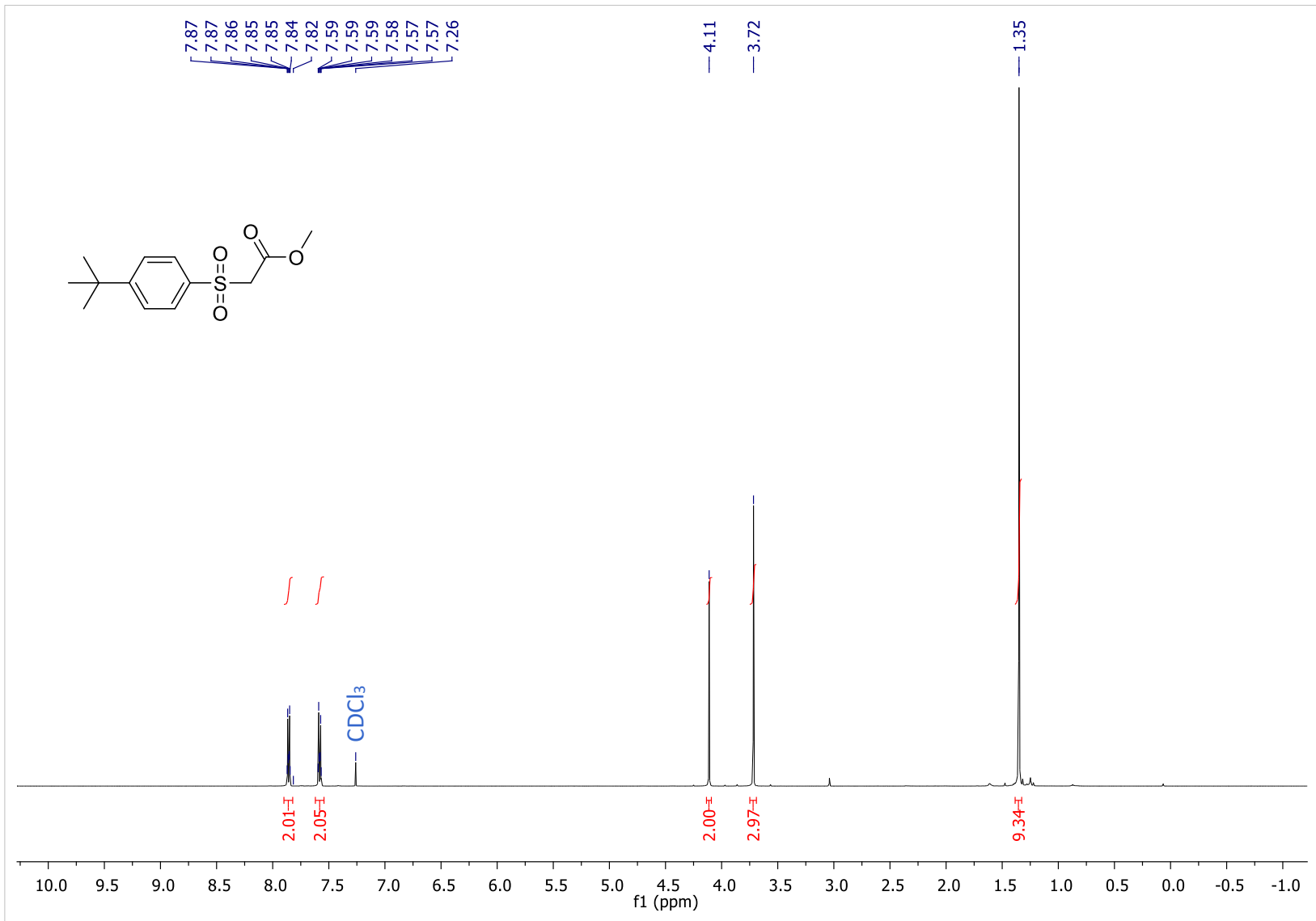

${ }^{13} \mathrm{C}\left\{{ }^{1} \mathrm{H}\right\}$ NMR (126 MHz, $\left.\mathrm{CDCl}_{3}\right)$

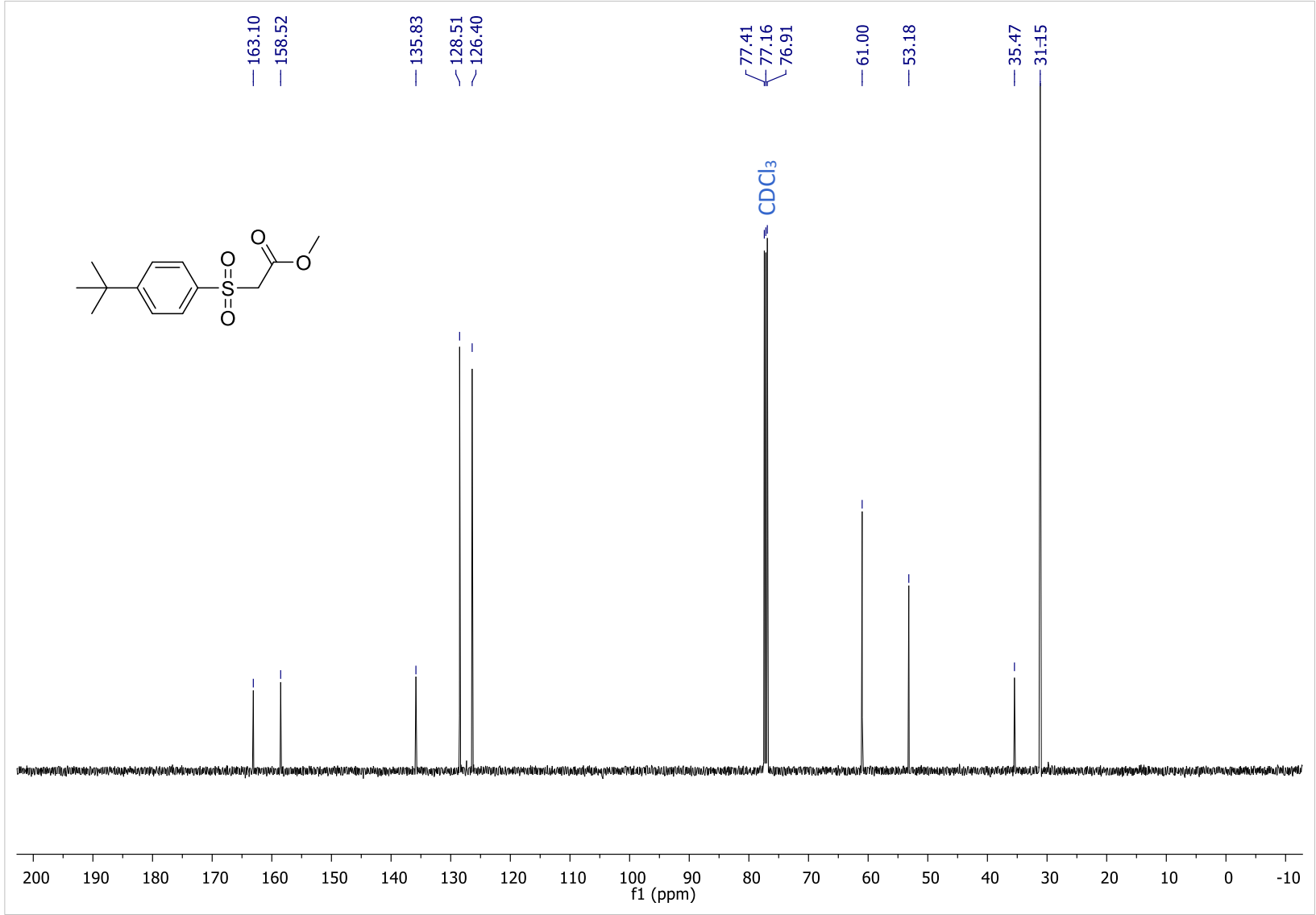


3db

${ }^{1} \mathrm{H} \mathrm{NMR}\left(500 \mathrm{MHz}, \mathrm{CDCl}_{3}\right)$

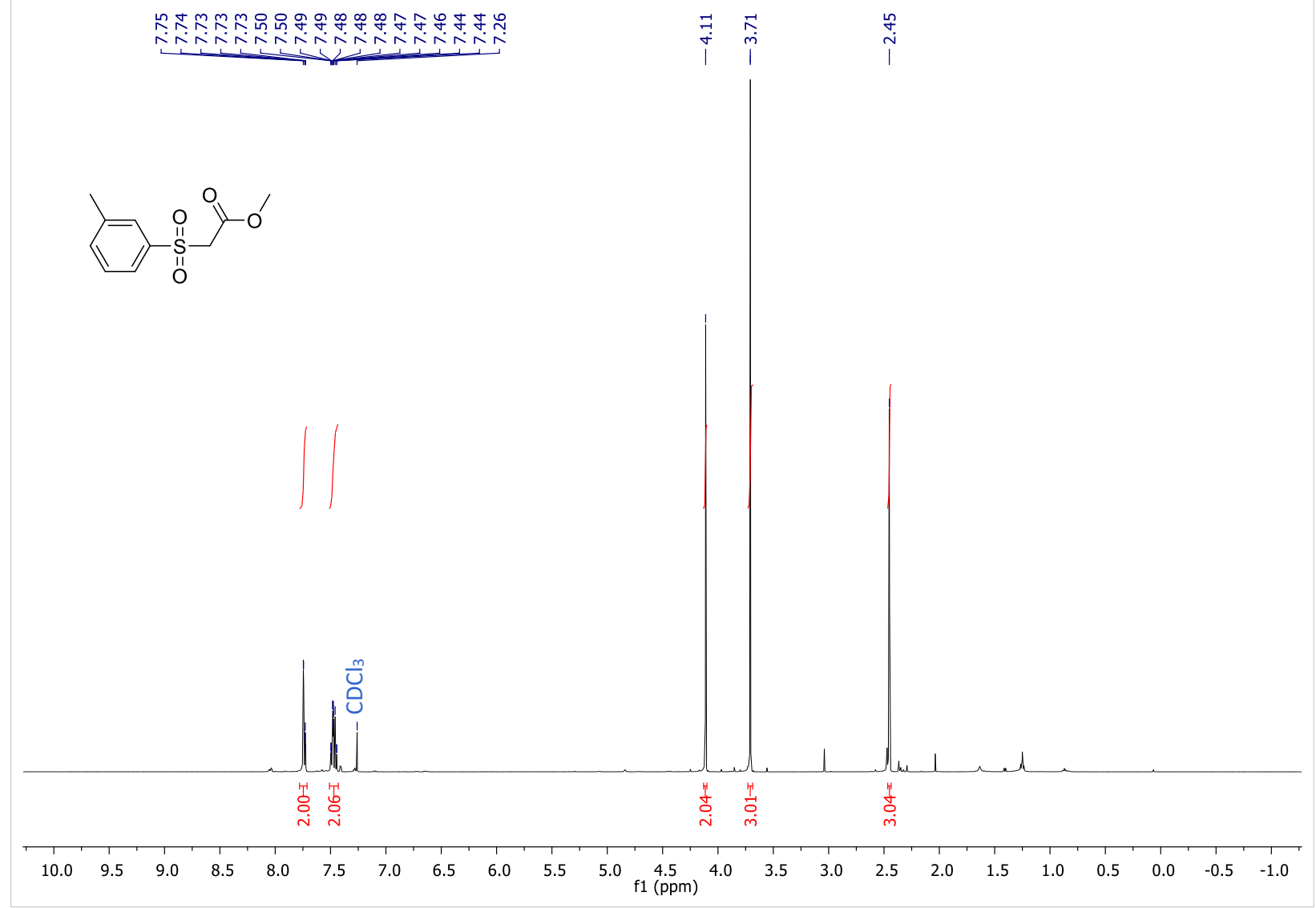

${ }^{13} \mathrm{C}\left\{{ }^{1} \mathrm{H}\right\}$ NMR $\left(126 \mathrm{MHz}, \mathrm{CDCl}_{3}\right)$

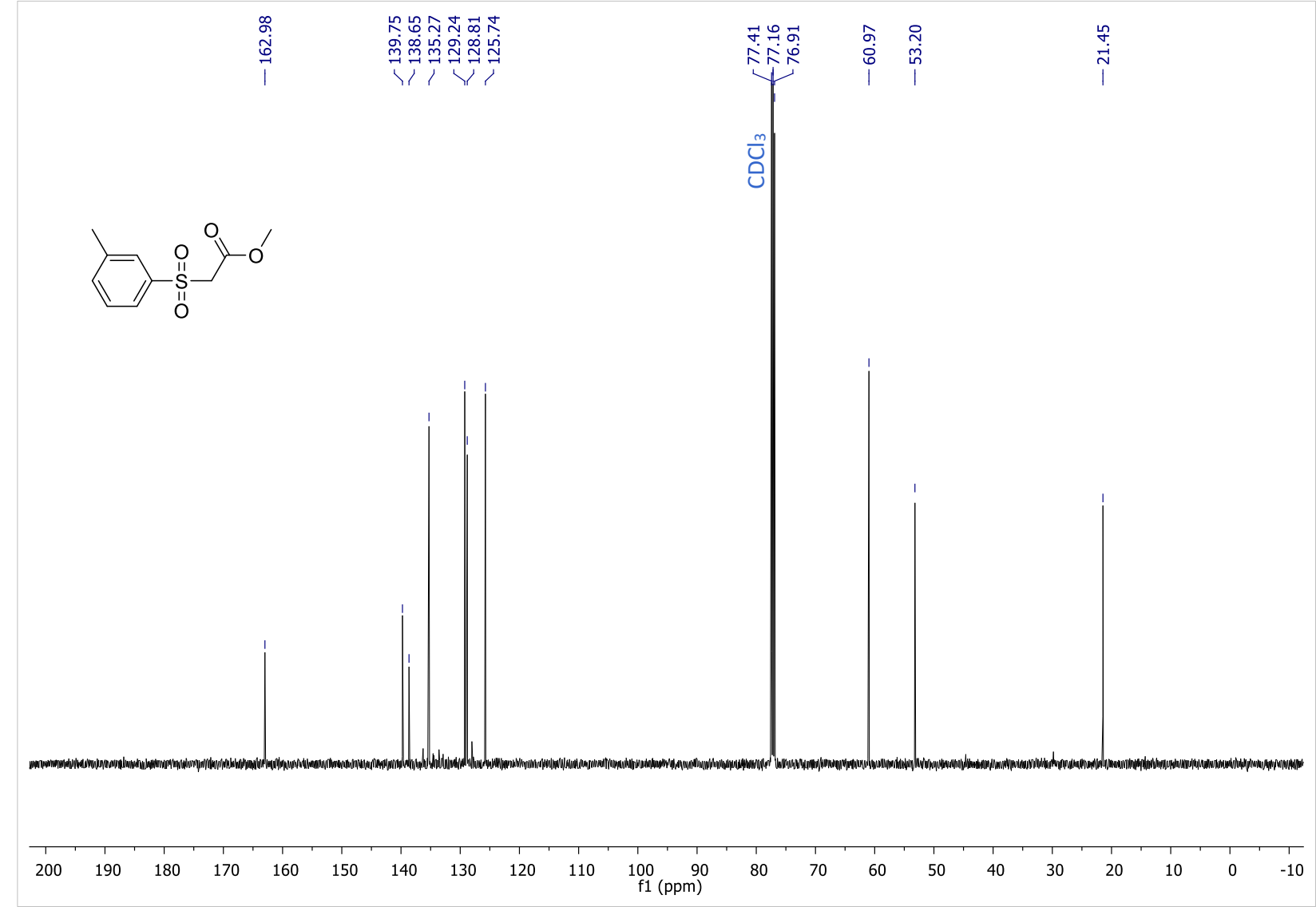


${ }^{1} \mathrm{H}$ NMR (500 MHz, $\mathrm{CDCl}_{3}$ )

3eb

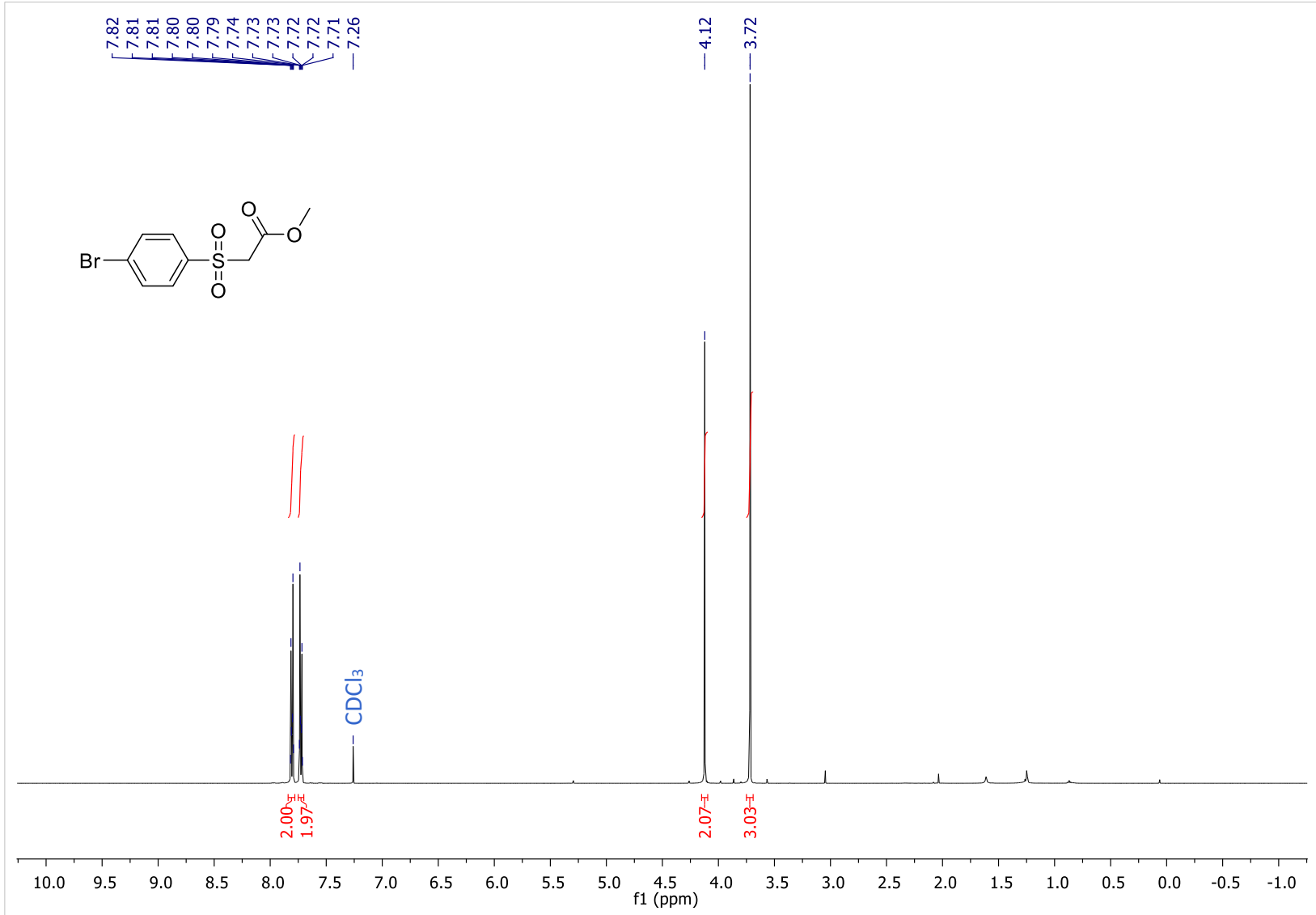

${ }^{13} \mathrm{C}\left\{{ }^{1} \mathrm{H}\right\}$ NMR $\left(126 \mathrm{MHz}, \mathrm{CDCl}_{3}\right)$

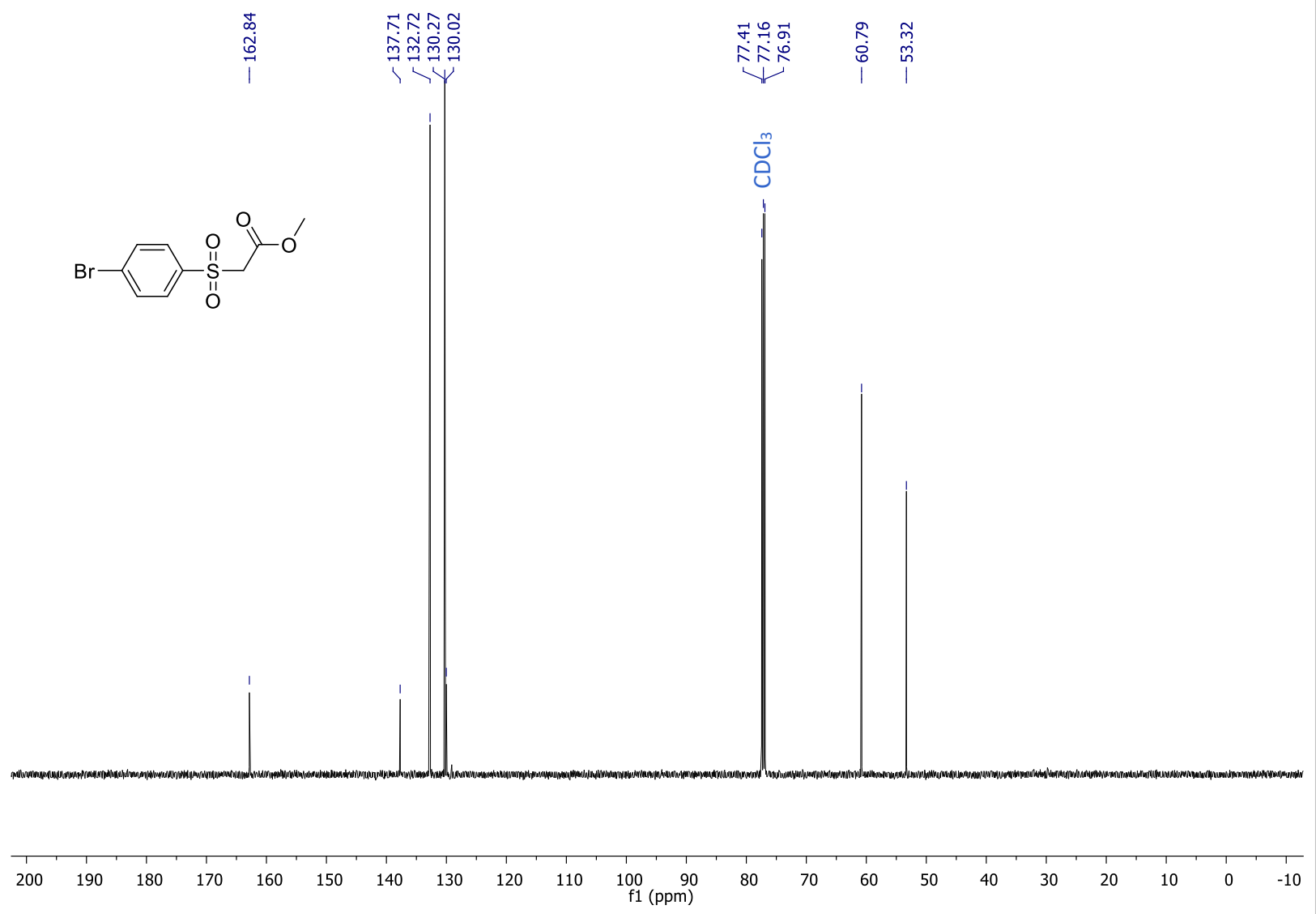


3fb

${ }^{1} \mathrm{H}$ NMR (500 MHz, $\left.\mathrm{CDCl}_{3}\right)$

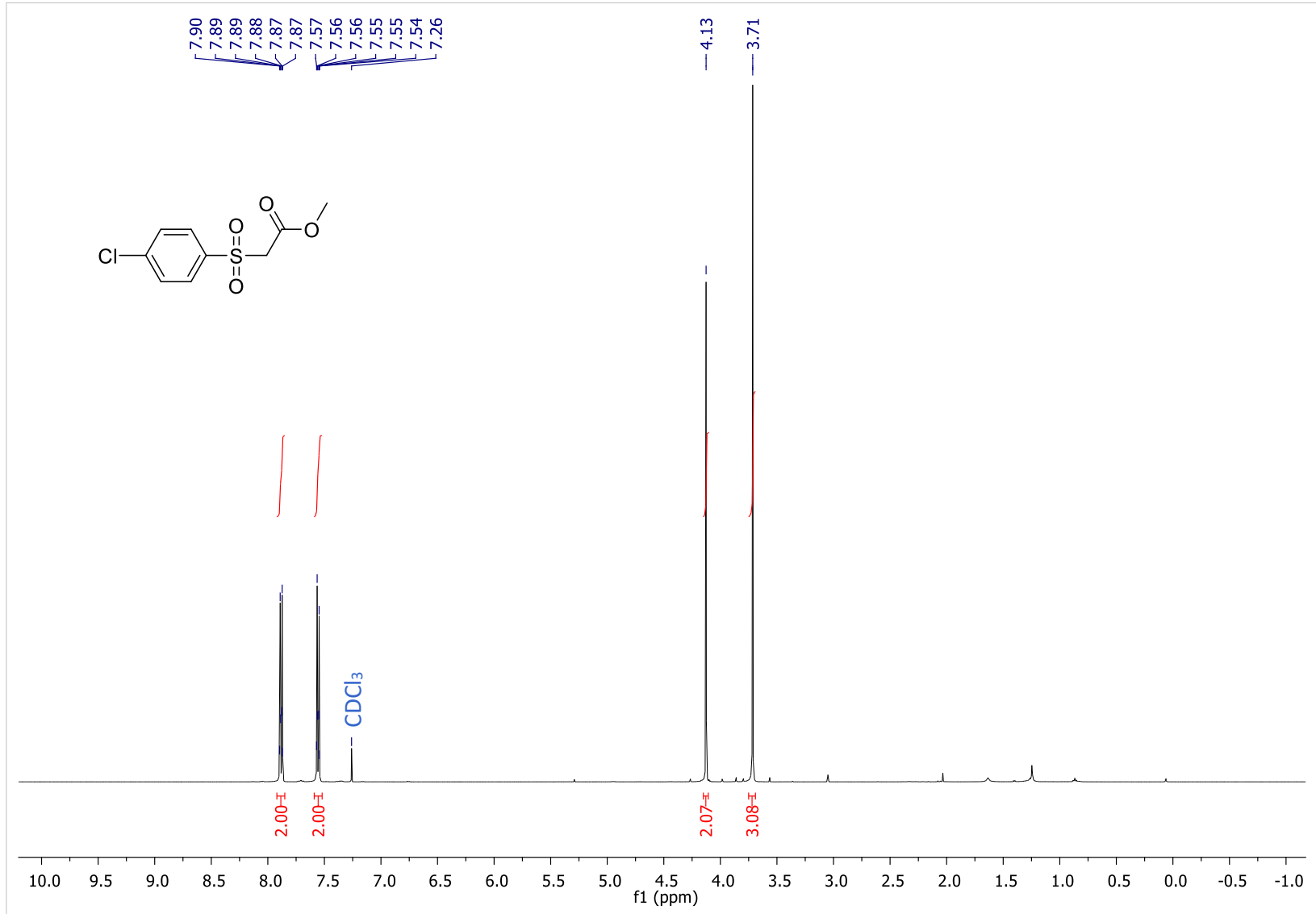

${ }^{13} \mathrm{C}\left\{{ }^{1} \mathrm{H}\right\}$ NMR (126 MHz, $\left.\mathrm{CDCl}_{3}\right)$

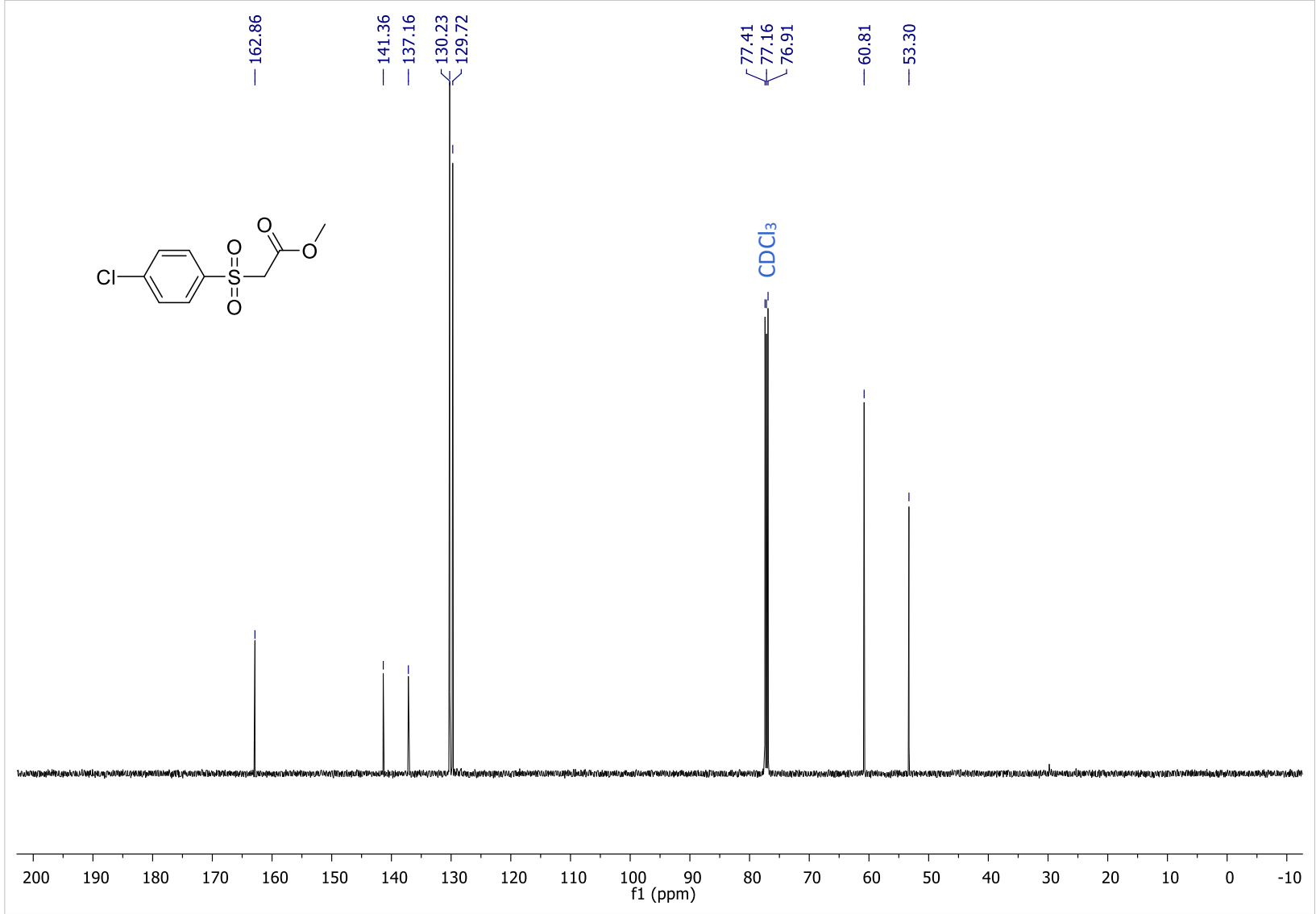


${ }^{1} \mathrm{H}$ NMR (500 MHz, $\mathrm{CDCl}_{3}$ )

3gb

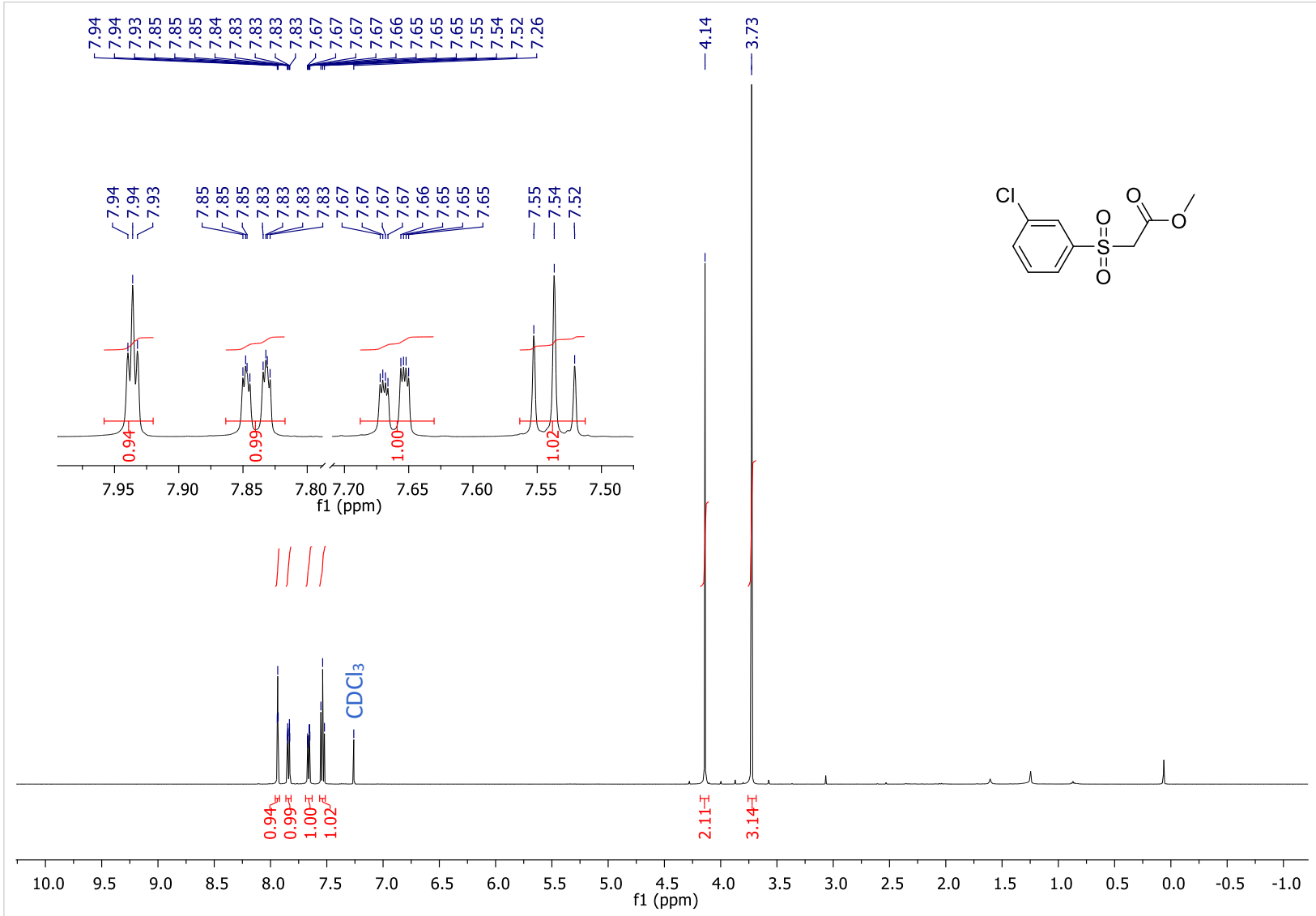

${ }^{13} \mathrm{C}\left\{{ }^{1} \mathrm{H}\right\}$ NMR $\left(126 \mathrm{MHz}, \mathrm{CDCl}_{3}\right)$

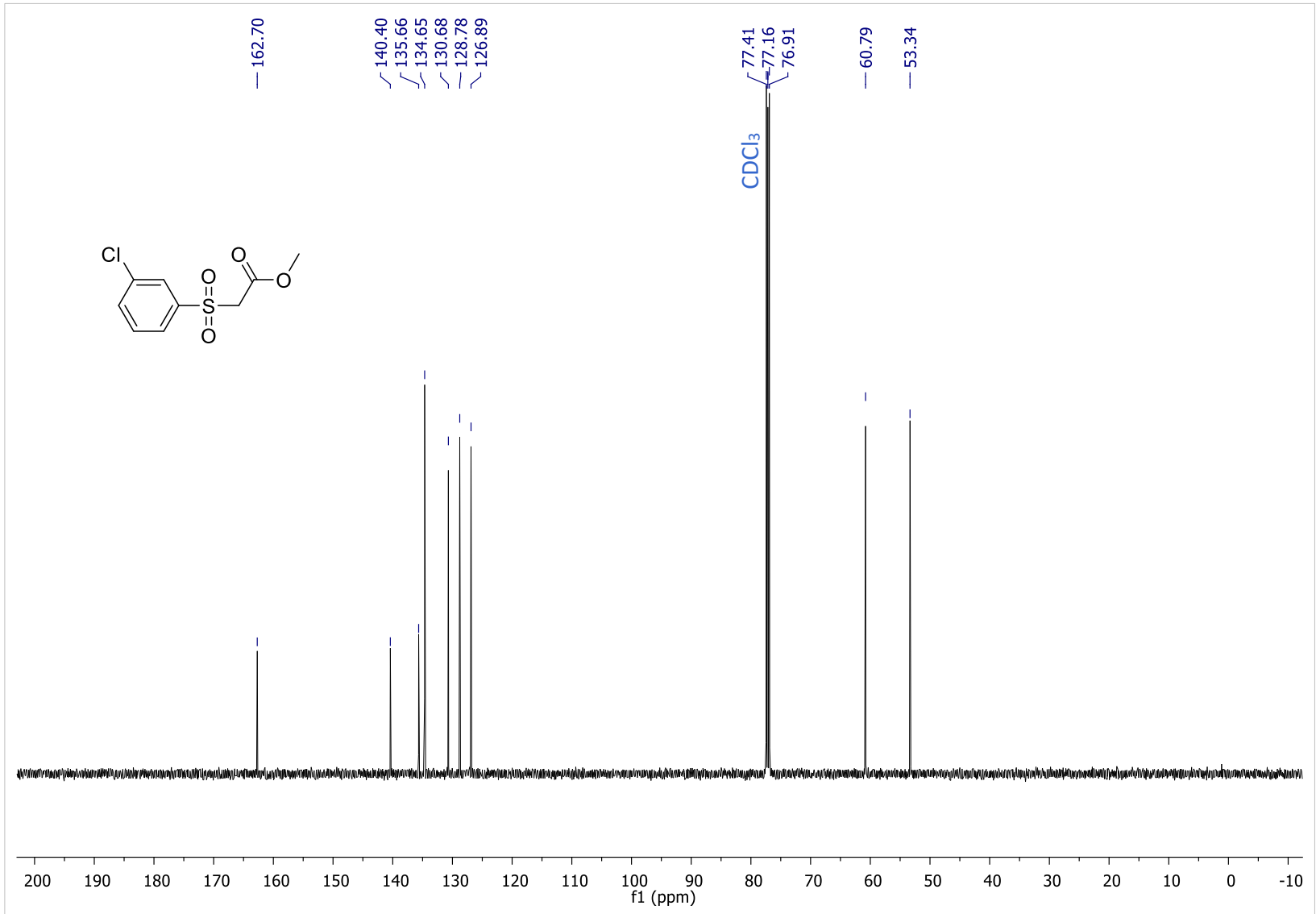


3hb

${ }^{1} \mathrm{H} \mathrm{NMR}\left(500 \mathrm{MHz}, \mathrm{CDCl}_{3}\right)$

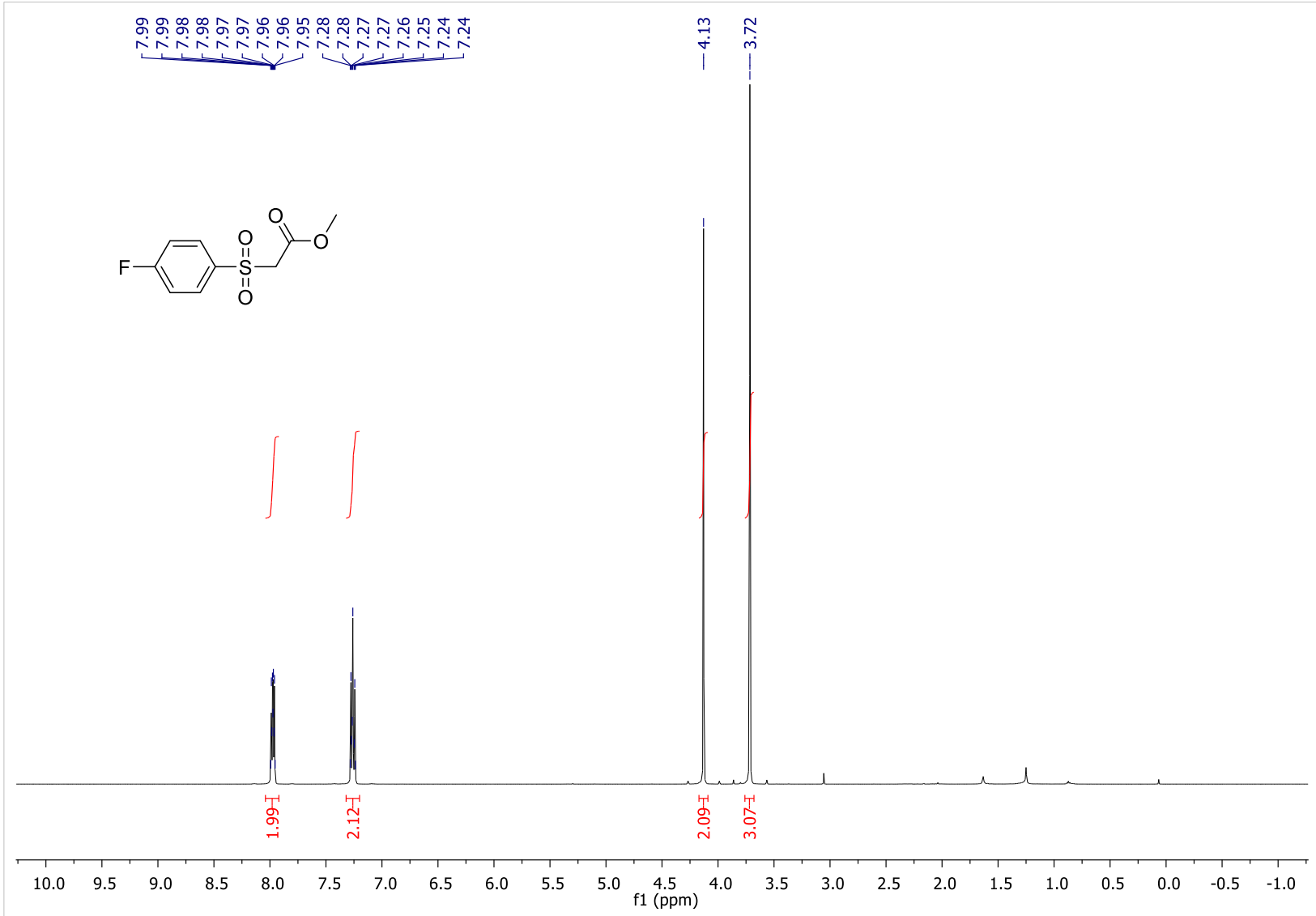

${ }^{13} \mathrm{C}\left\{{ }^{1} \mathrm{H}\right\}$ NMR (126 MHz, $\left.\mathrm{CDCl}_{3}\right)$

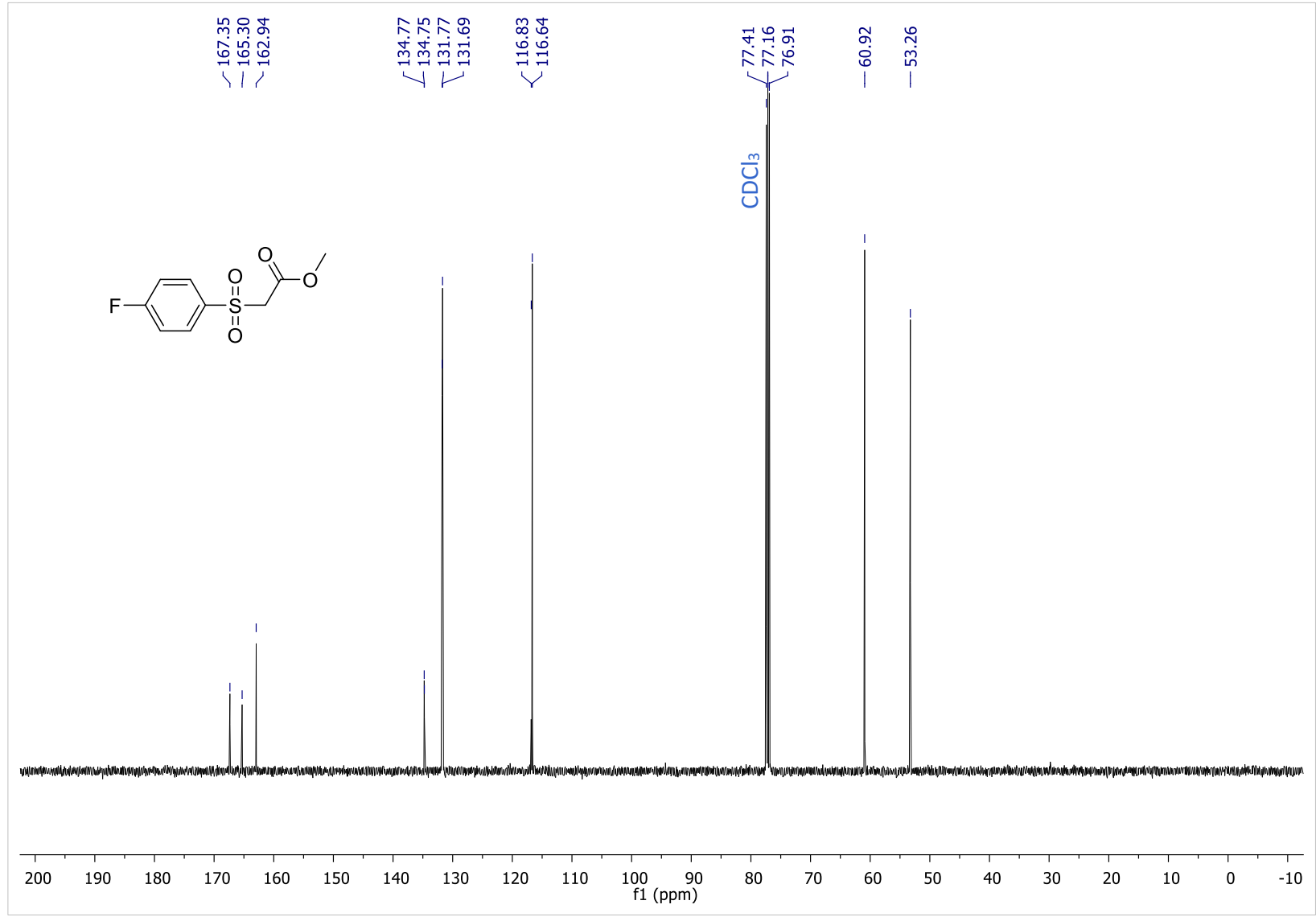


${ }^{1} \mathrm{H} \mathrm{NMR}\left(500 \mathrm{MHz}, \mathrm{CDCl}_{3}\right)$

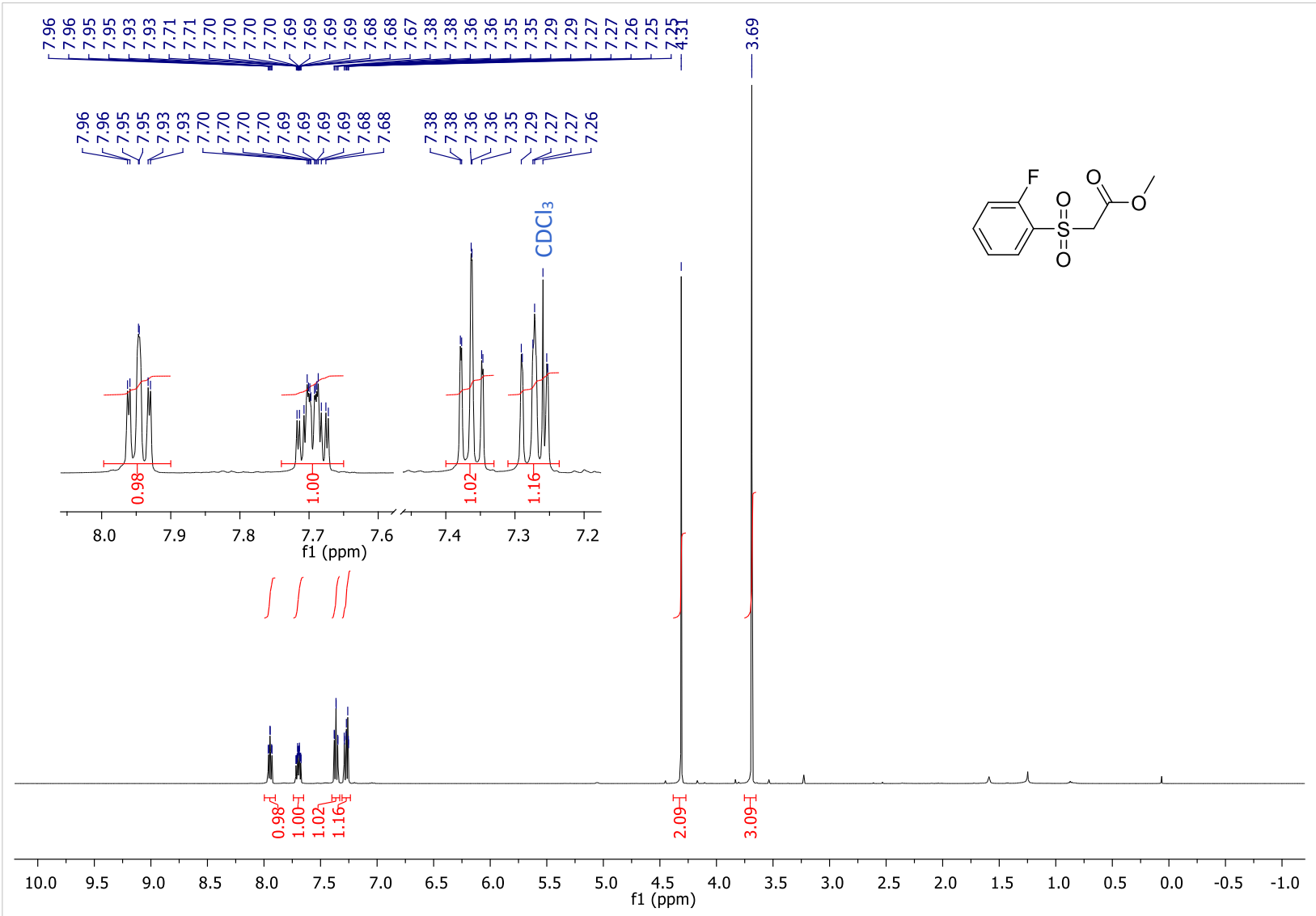

${ }^{13} \mathrm{C}\left\{{ }^{1} \mathrm{H}\right\}$ NMR (126 MHz, $\left.\mathrm{CDCl}_{3}\right)$

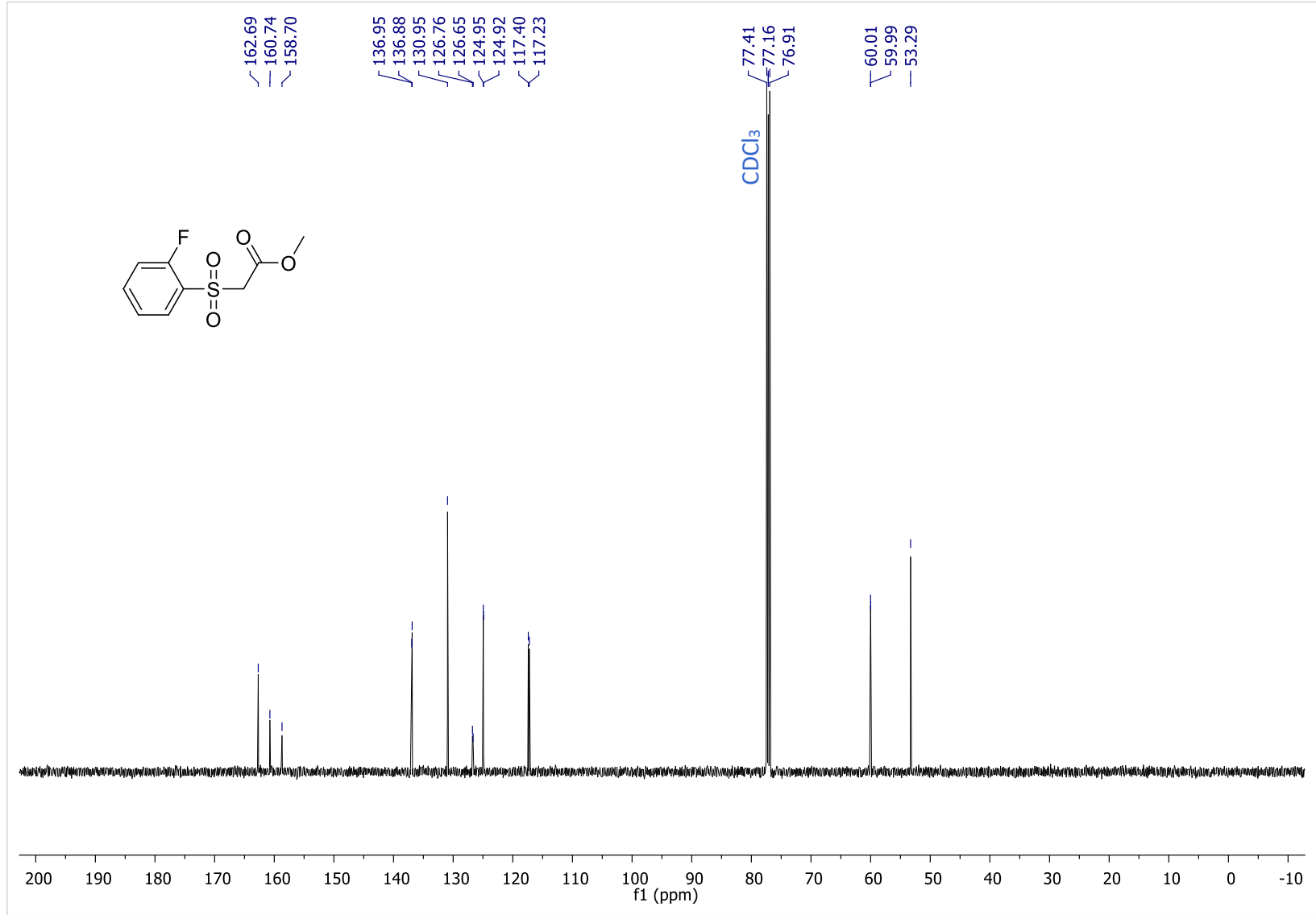


${ }^{1} \mathrm{H}$ NMR $\left(500 \mathrm{MHz}, \mathrm{CDCl}_{3}\right)$

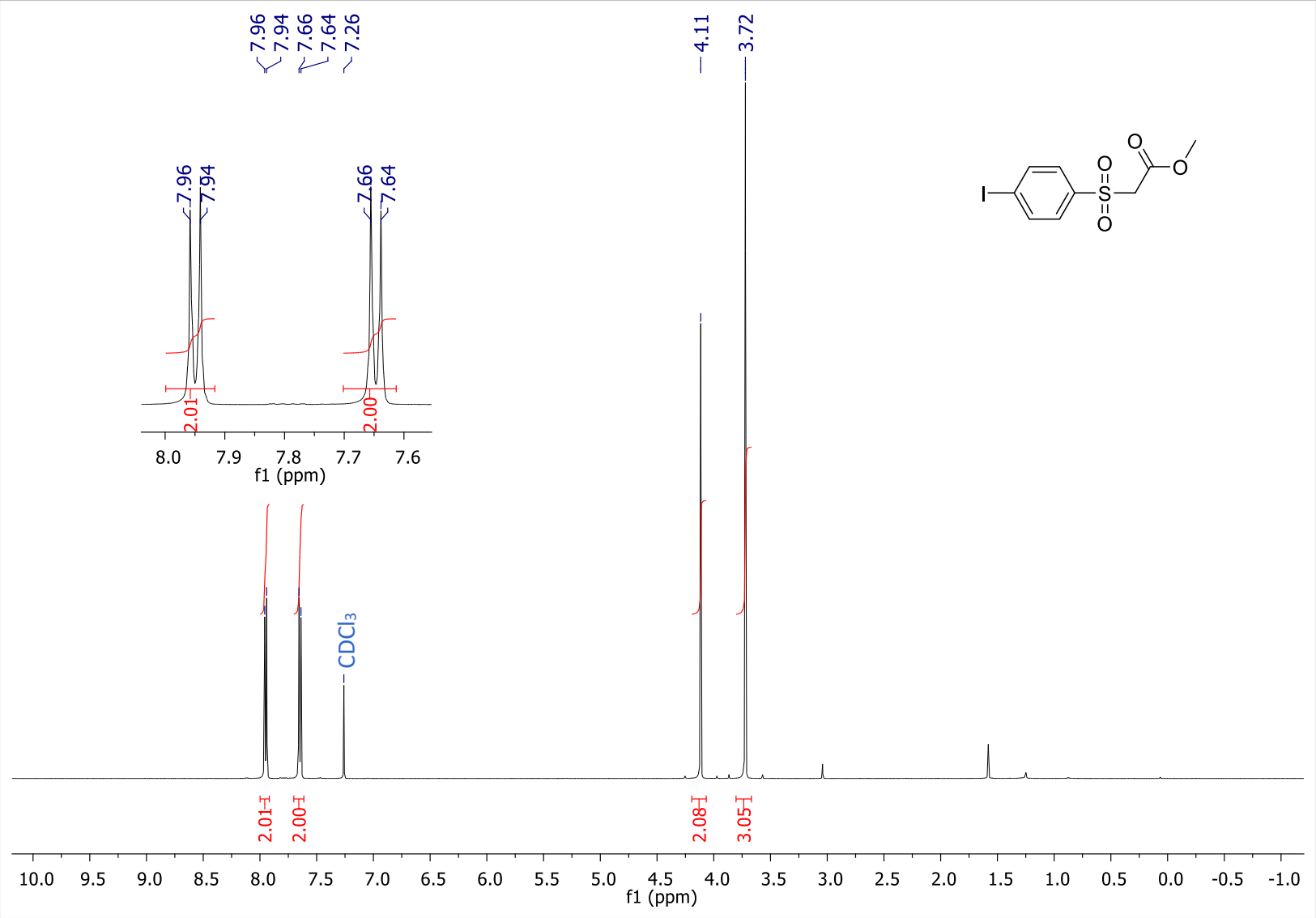

${ }^{13} \mathrm{C}\left\{{ }^{1} \mathrm{H}\right\}$ NMR $\left(126 \mathrm{MHz}, \mathrm{CDCl}_{3}\right)$

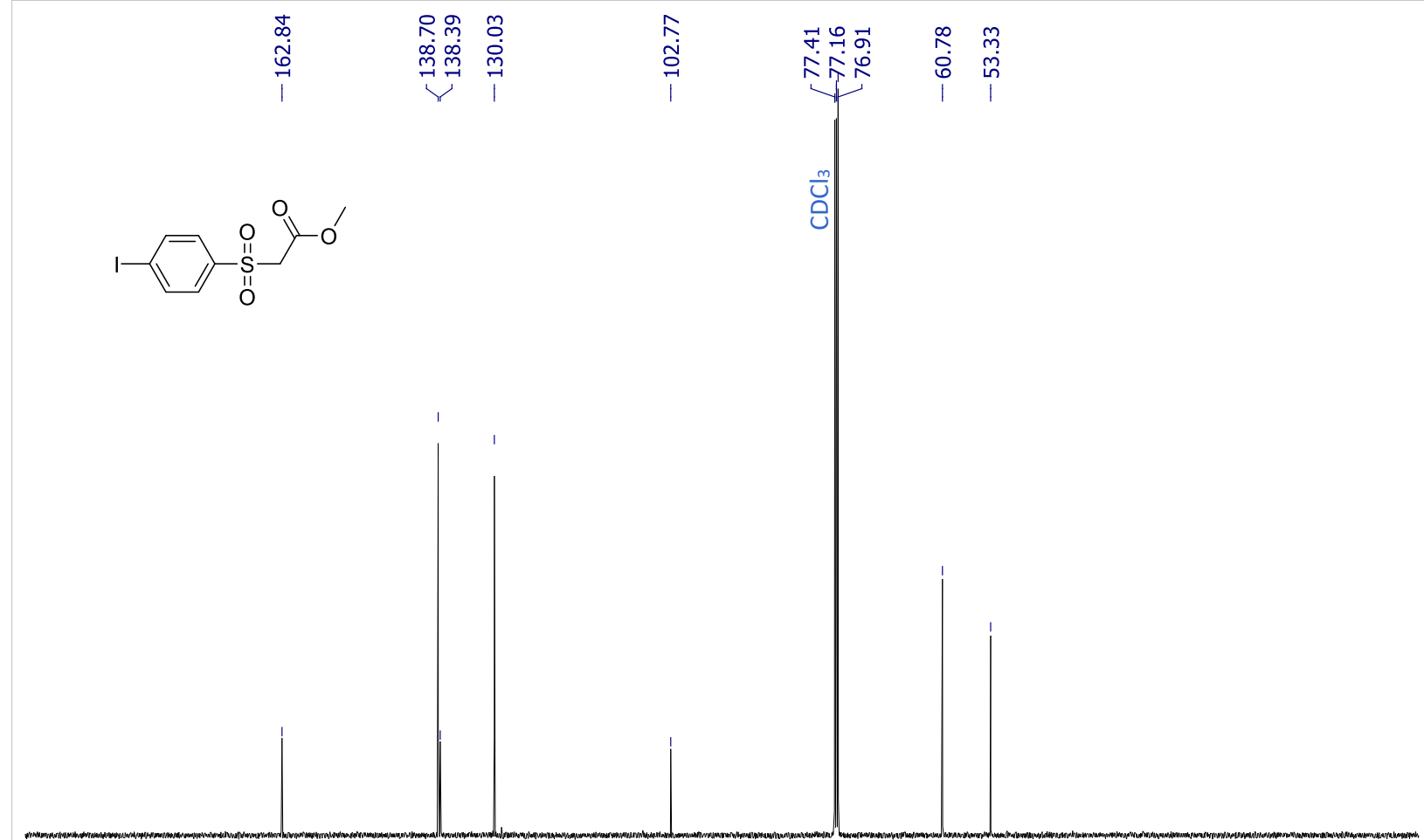

$\begin{array}{llllllllllllllllllllllll}200 & 190 & 180 & 170 & 160 & 150 & 140 & 130 & 120 & 110 & 100 & 90 & 80 & 70 & 60 & 50 & 40 & 30 & 20 & 10 & 0 & -10\end{array}$ 
$3 \mathbf{k b}$

${ }^{1} \mathrm{H}$ NMR (500 MHz, $\mathrm{CDCl}_{3}$ )

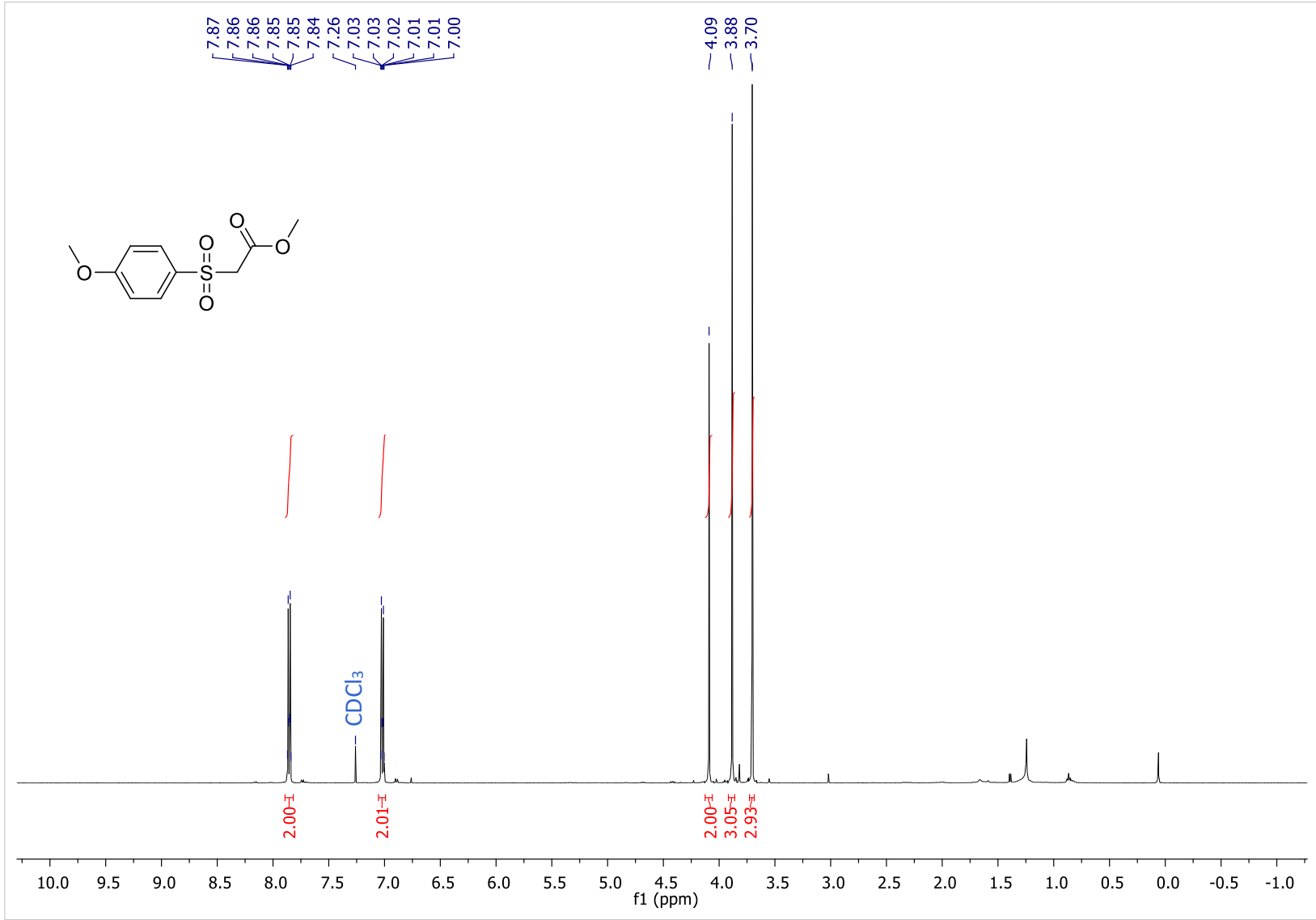

${ }^{13} \mathrm{C}\left\{{ }^{1} \mathrm{H}\right\}$ NMR $\left(126 \mathrm{MHz}, \mathrm{CDCl}_{3}\right)$

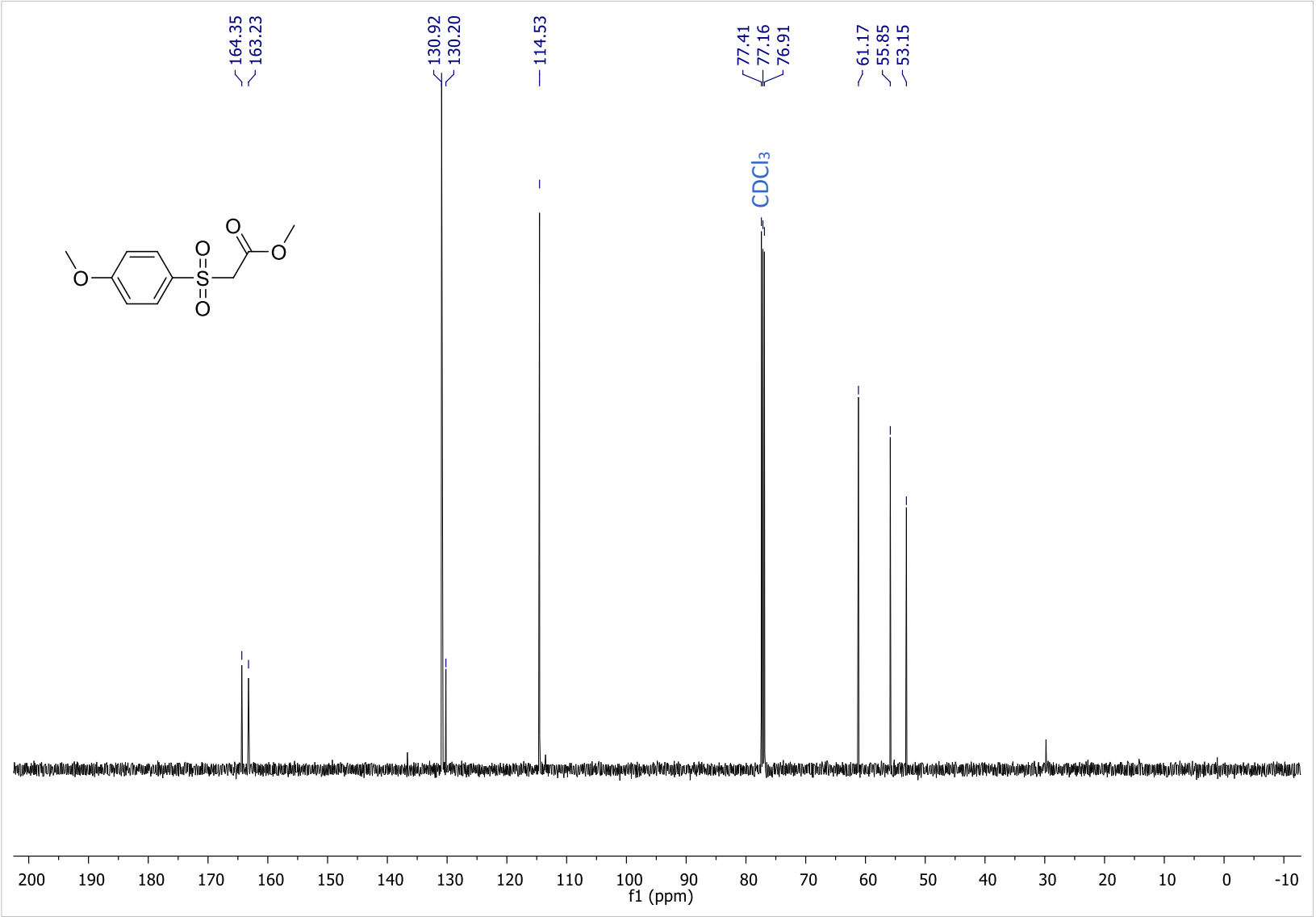


${ }^{1} \mathrm{H}$ NMR (500 MHz, $\mathrm{CDCl}_{3}$ )

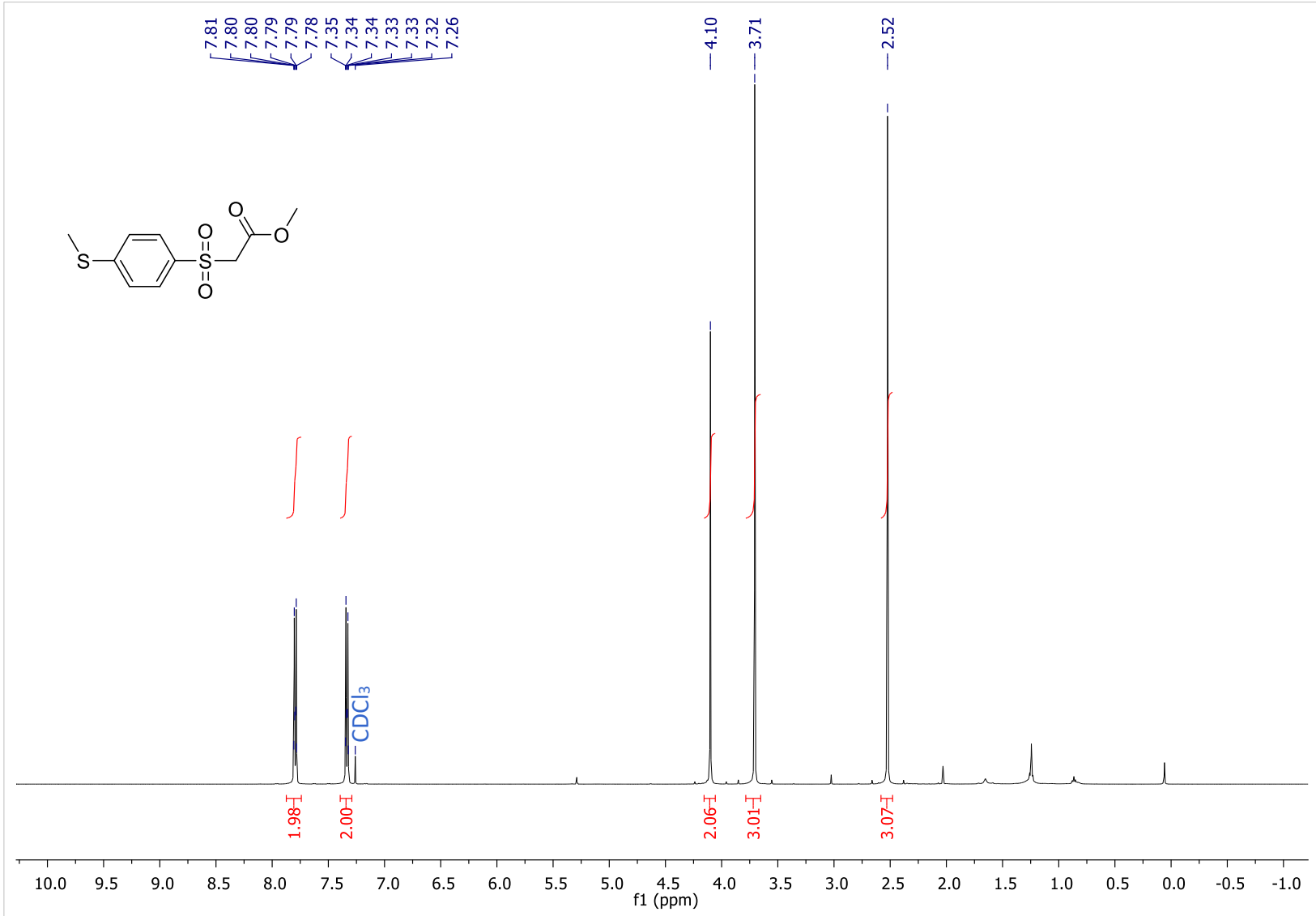

${ }^{13} \mathrm{C}\left\{{ }^{1} \mathrm{H}\right\}$ NMR $\left(126 \mathrm{MHz}, \mathrm{CDCl}_{3}\right)$

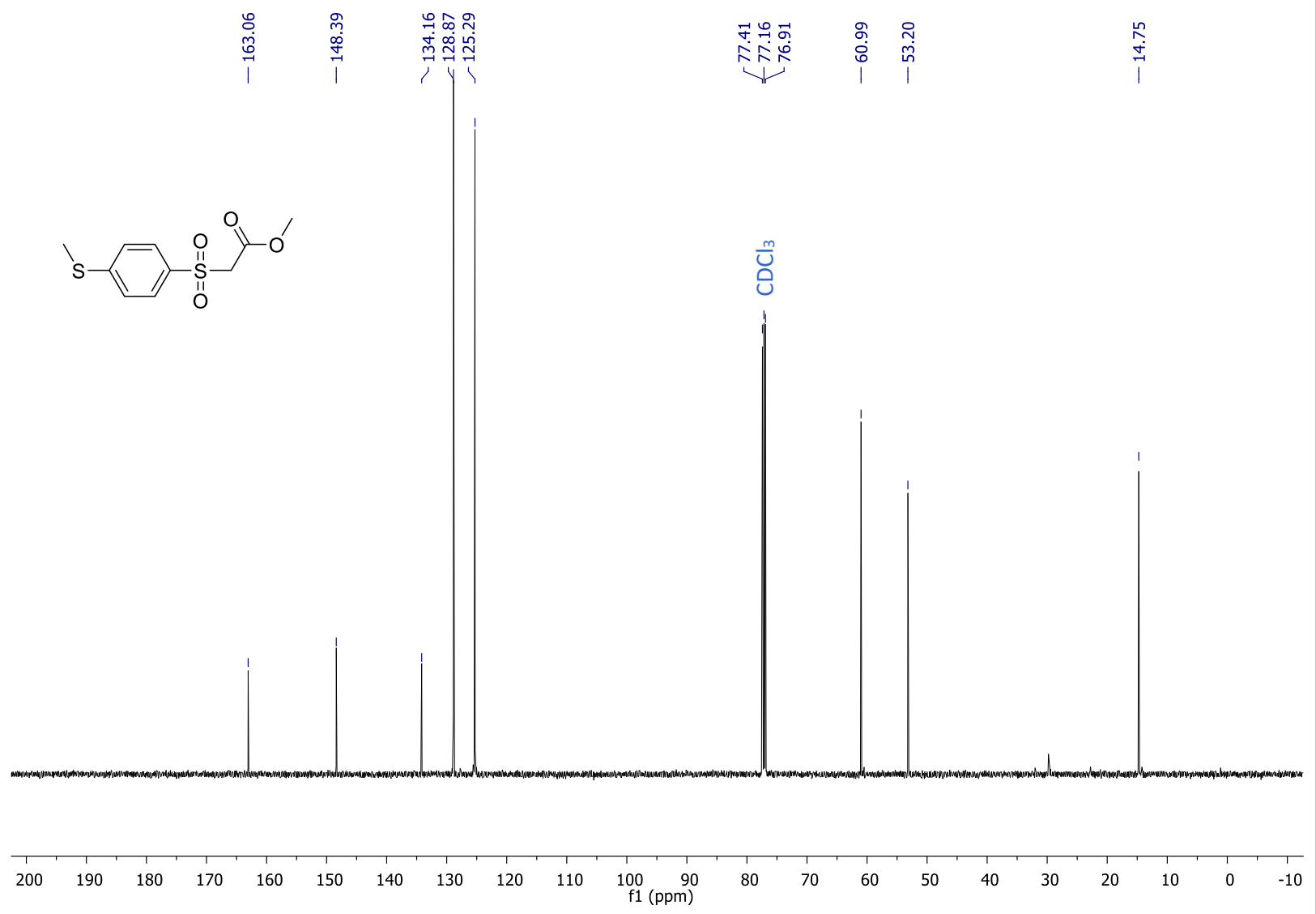


${ }^{1} \mathrm{H}$ NMR (500 MHz, DMSO- $\left.d_{6}\right)$

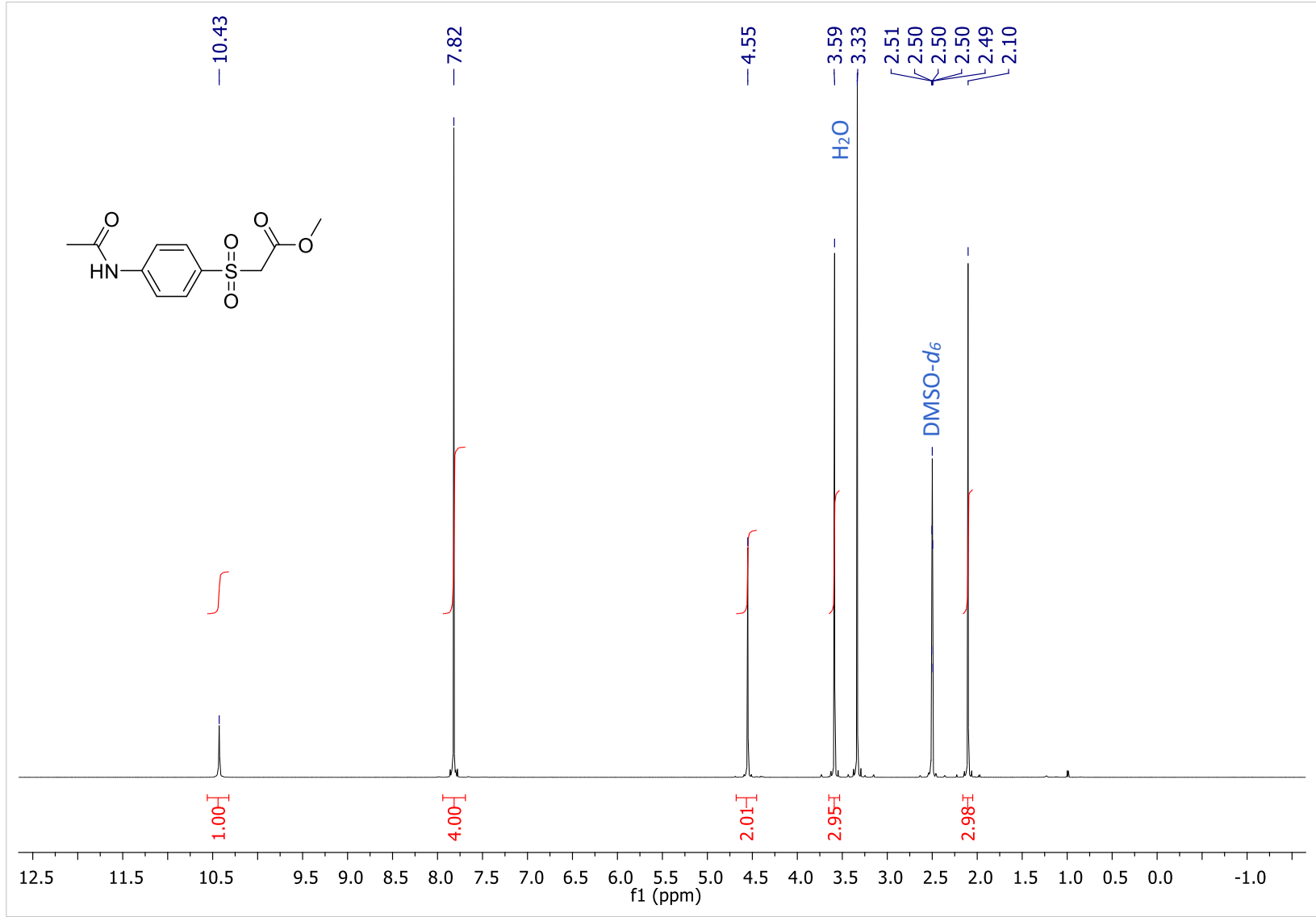

${ }^{13} \mathrm{C}\left\{{ }^{1} \mathrm{H}\right\}$ NMR (126 MHz, DMSO- $\left.d_{6}\right)$

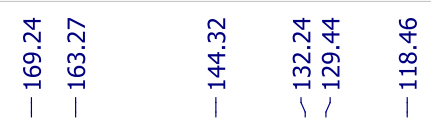

\section{$3 \mathrm{mb}$}

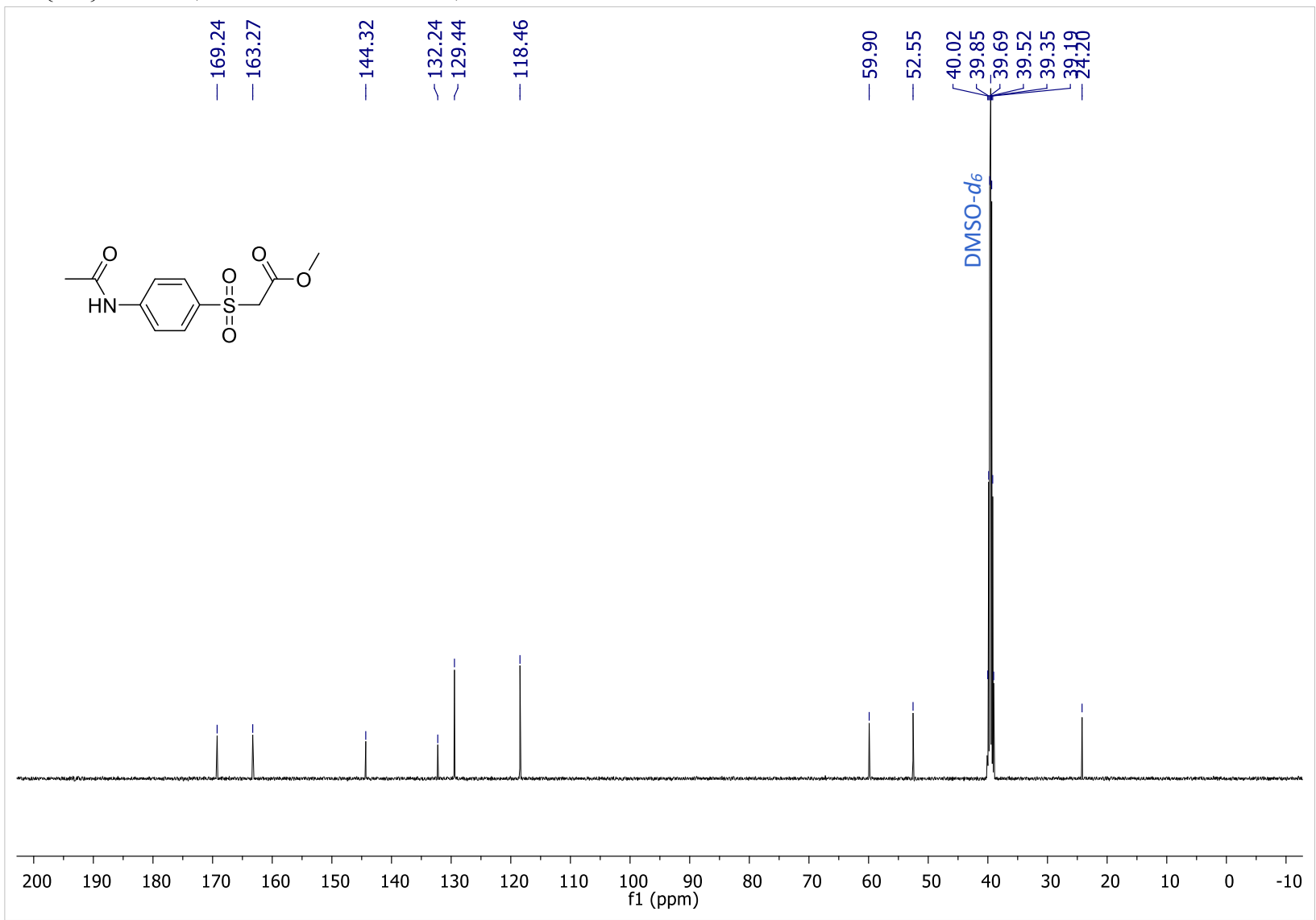


3nb

${ }^{1} \mathrm{H} \mathrm{NMR}\left(500 \mathrm{MHz}, \mathrm{CDCl}_{3}\right)$

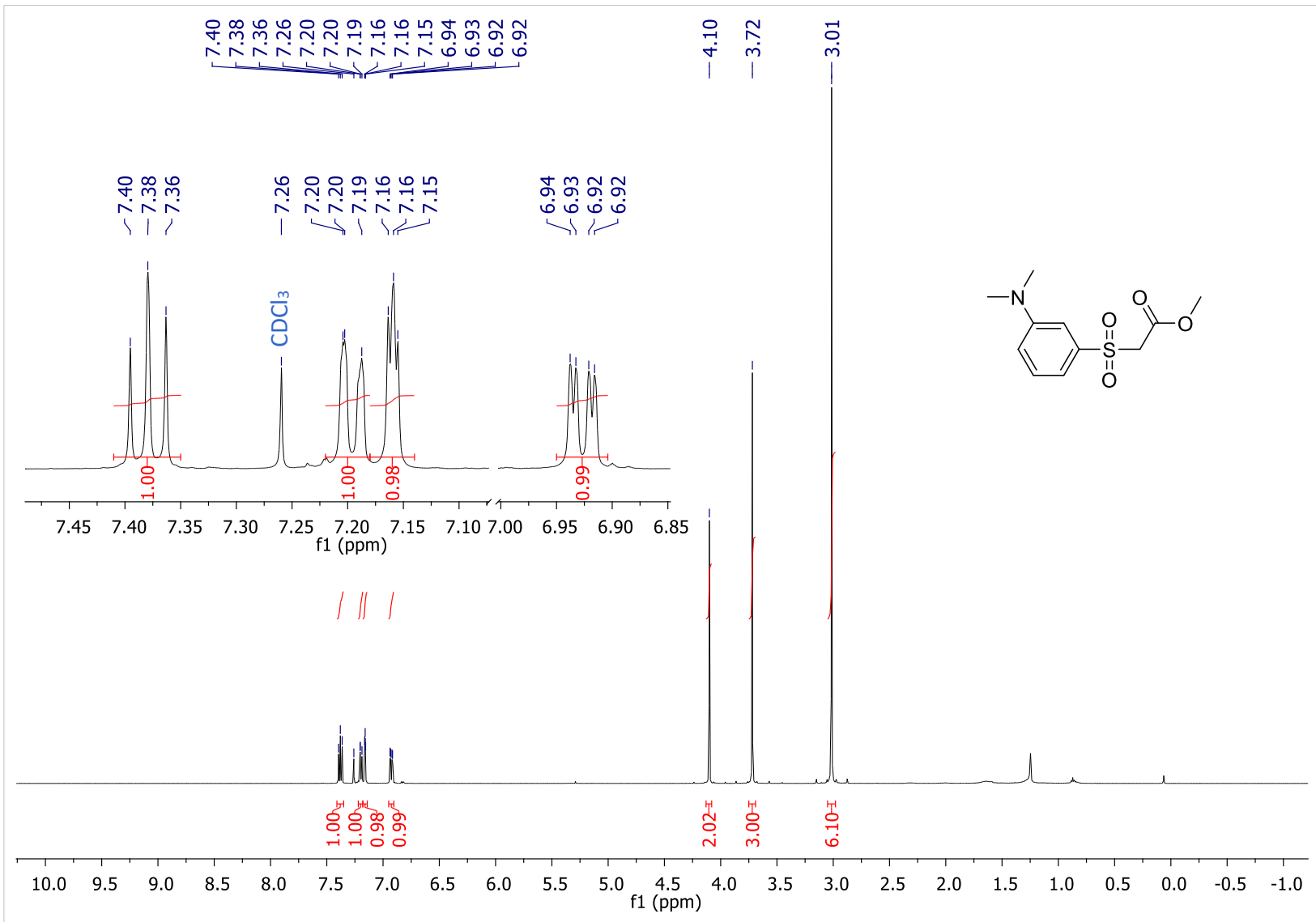

${ }^{13} \mathrm{C}\left\{{ }^{1} \mathrm{H}\right\}$ NMR (126 MHz, $\left.\mathrm{CDCl}_{3}\right)$

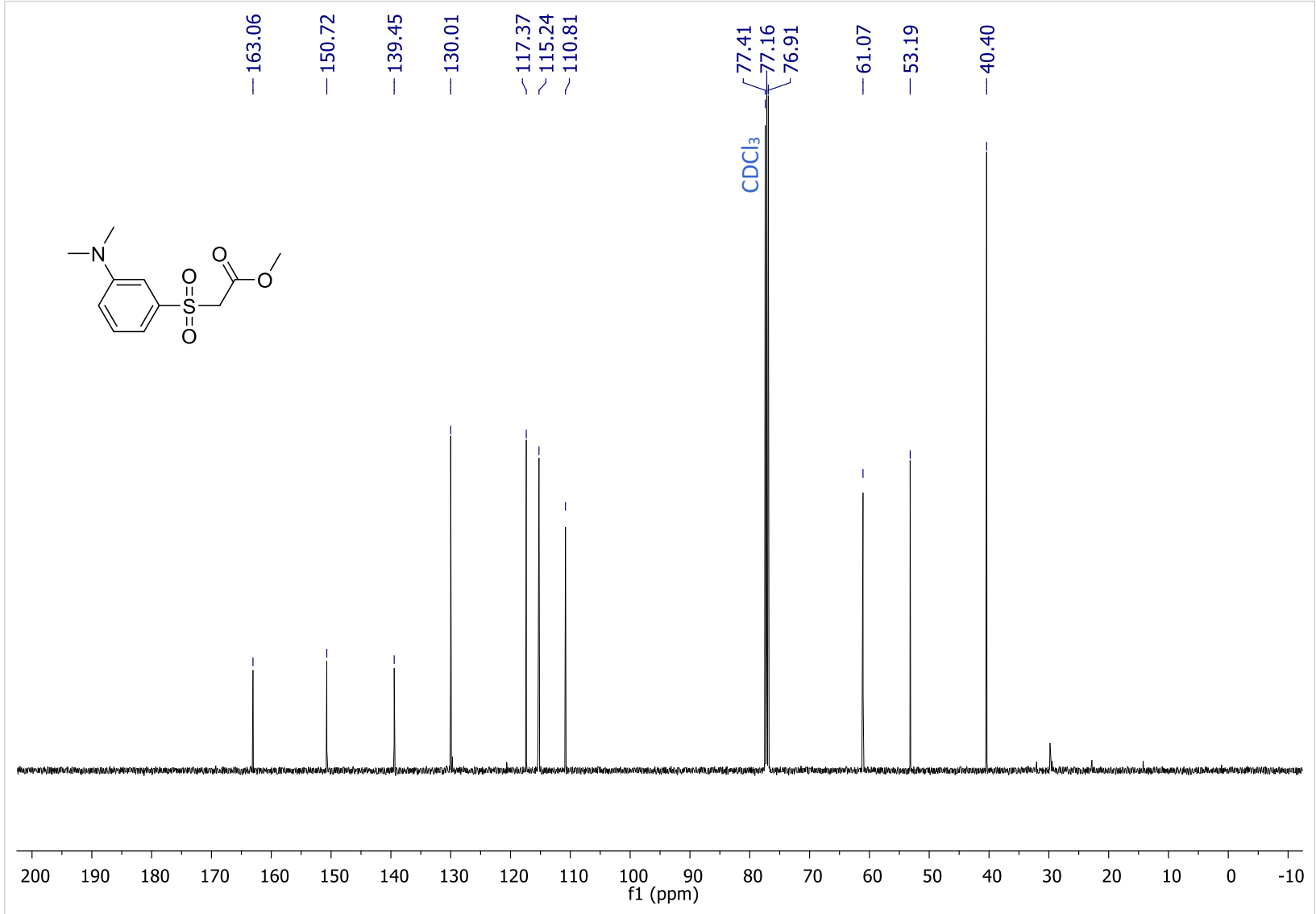


${ }^{1} \mathrm{H} \mathrm{NMR}\left(500 \mathrm{MHz}, \mathrm{CDCl}_{3}\right)$

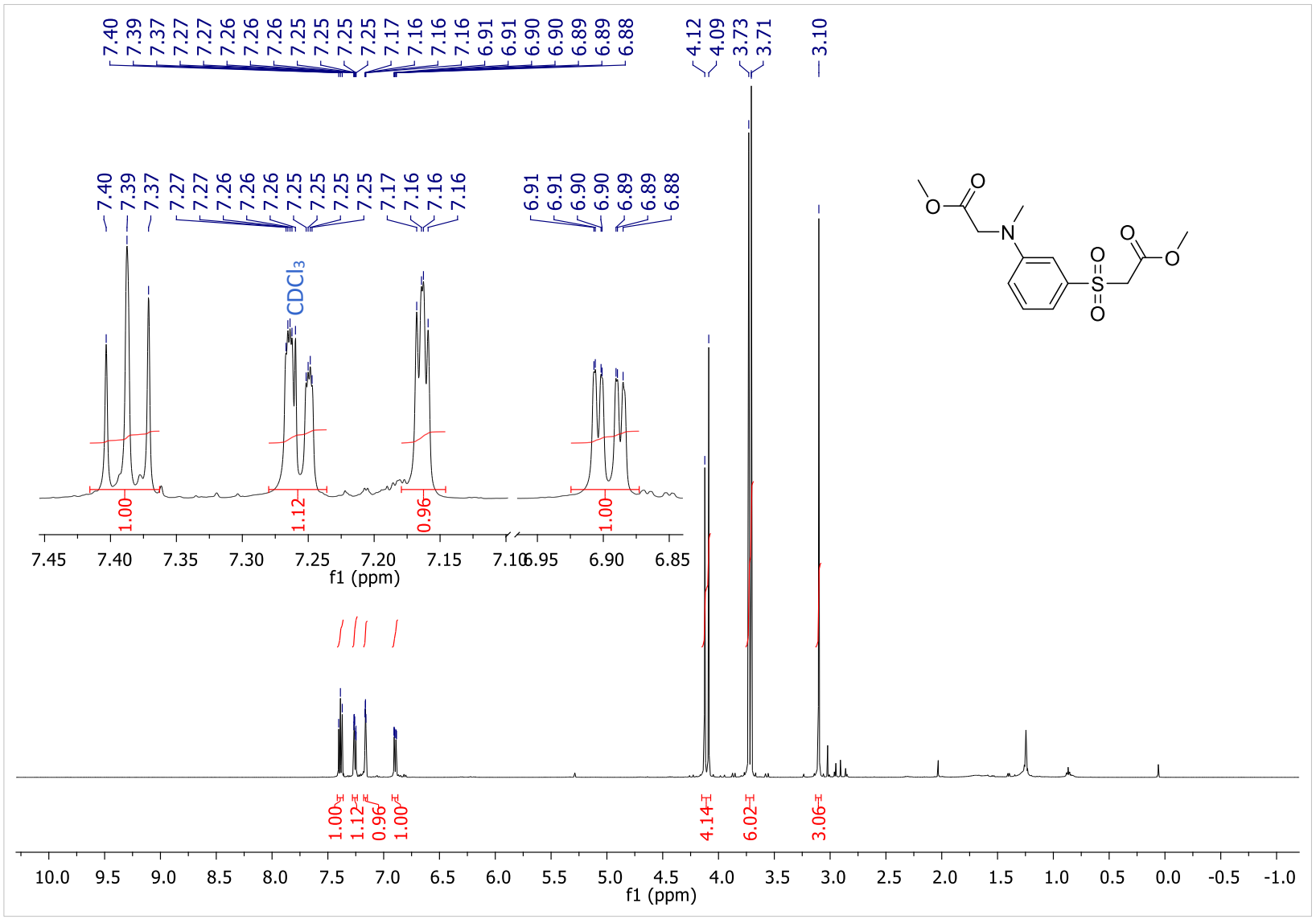

${ }^{13} \mathrm{C}\left\{{ }^{1} \mathrm{H}\right\} \mathrm{NMR}\left(126 \mathrm{MHz}, \mathrm{CDCl}_{3}\right)$

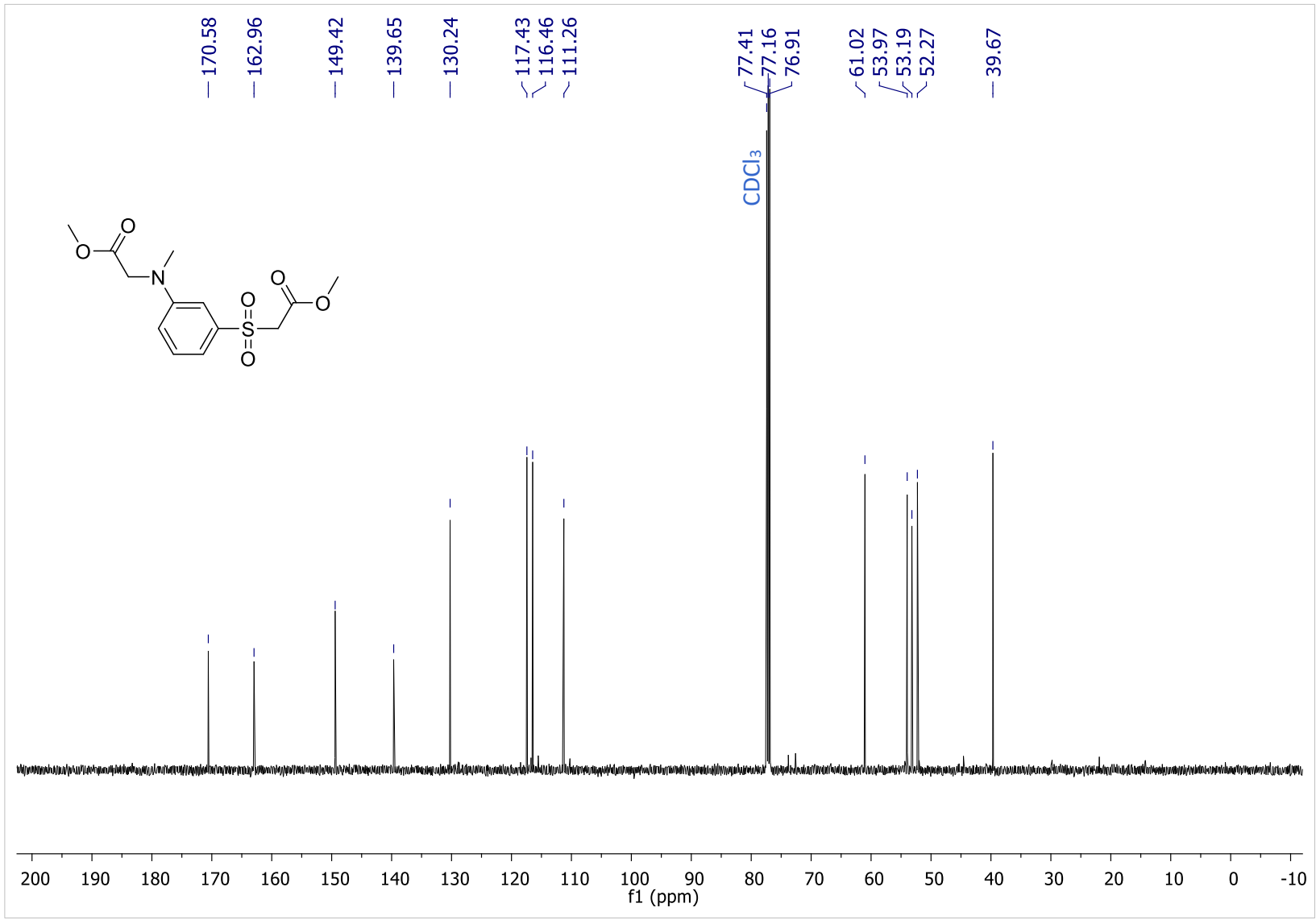


${ }^{1} \mathrm{H}$ NMR (500 MHz, $\mathrm{CDCl}_{3}$ )

3ob

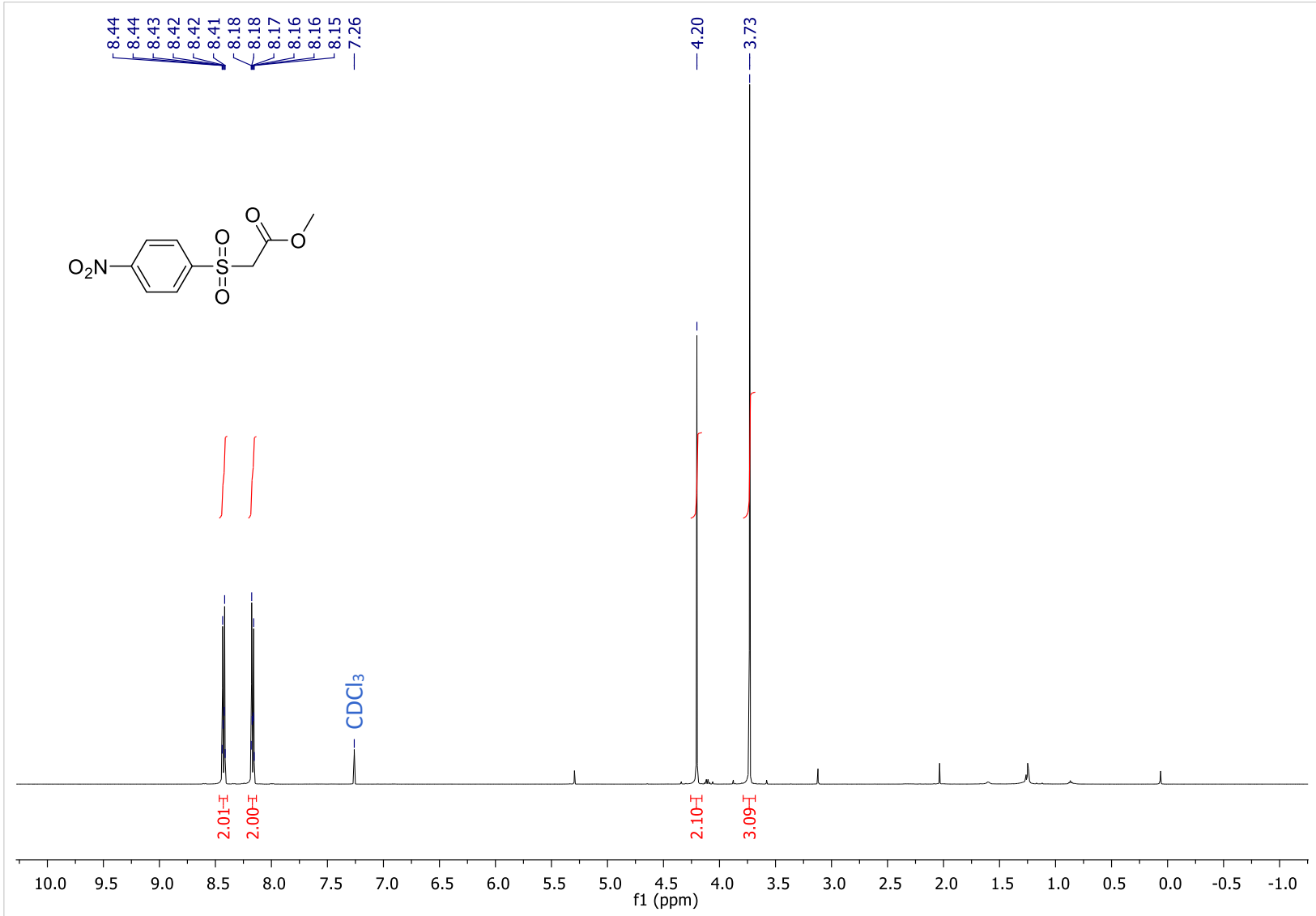

${ }^{13} \mathrm{C}\left\{{ }^{1} \mathrm{H}\right\}$ NMR $\left(126 \mathrm{MHz}, \mathrm{CDCl}_{3}\right)$

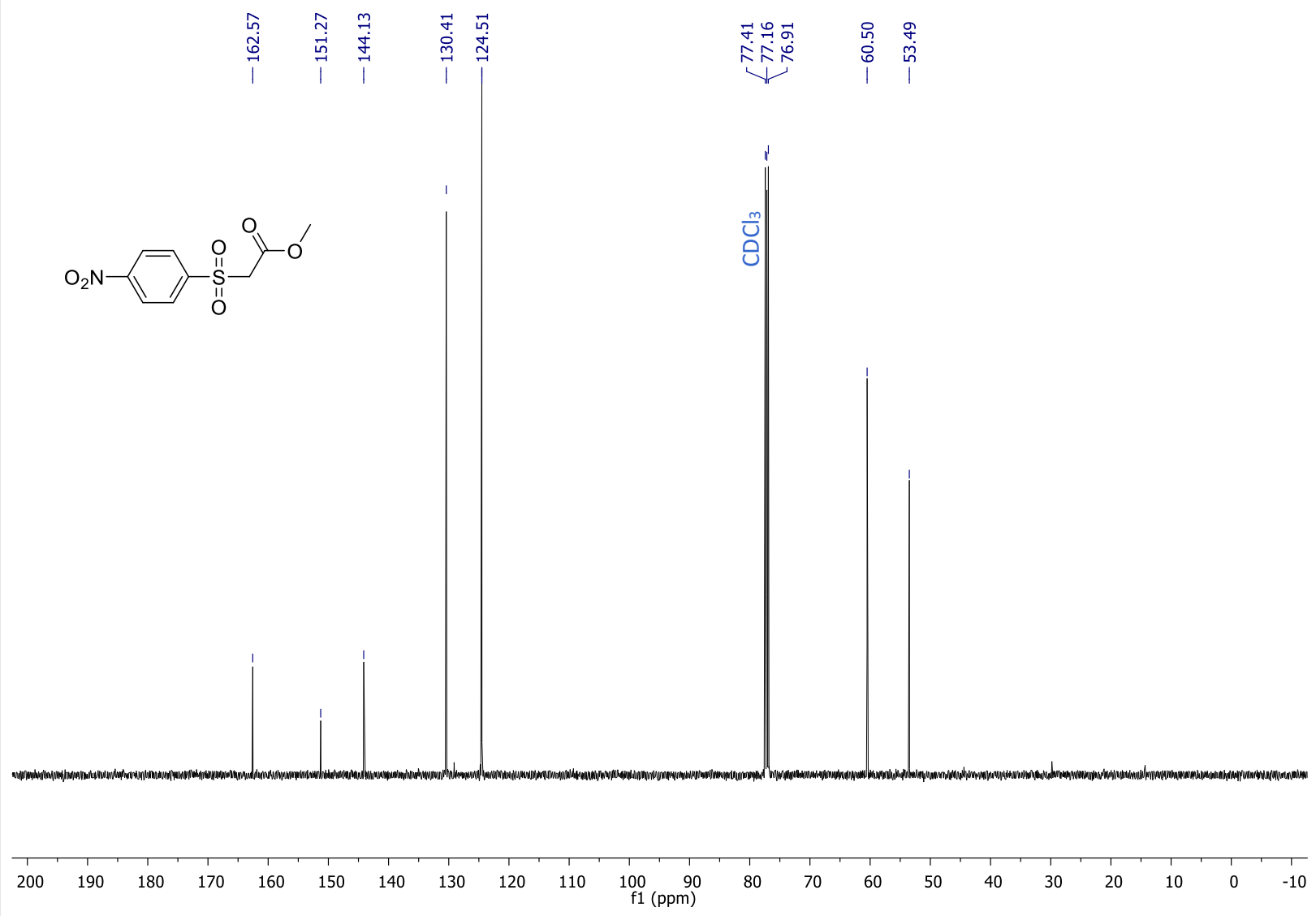


${ }^{1} \mathrm{H}$ NMR (500 MHz, $\mathrm{CDCl}_{3}$ )

\section{3pb}

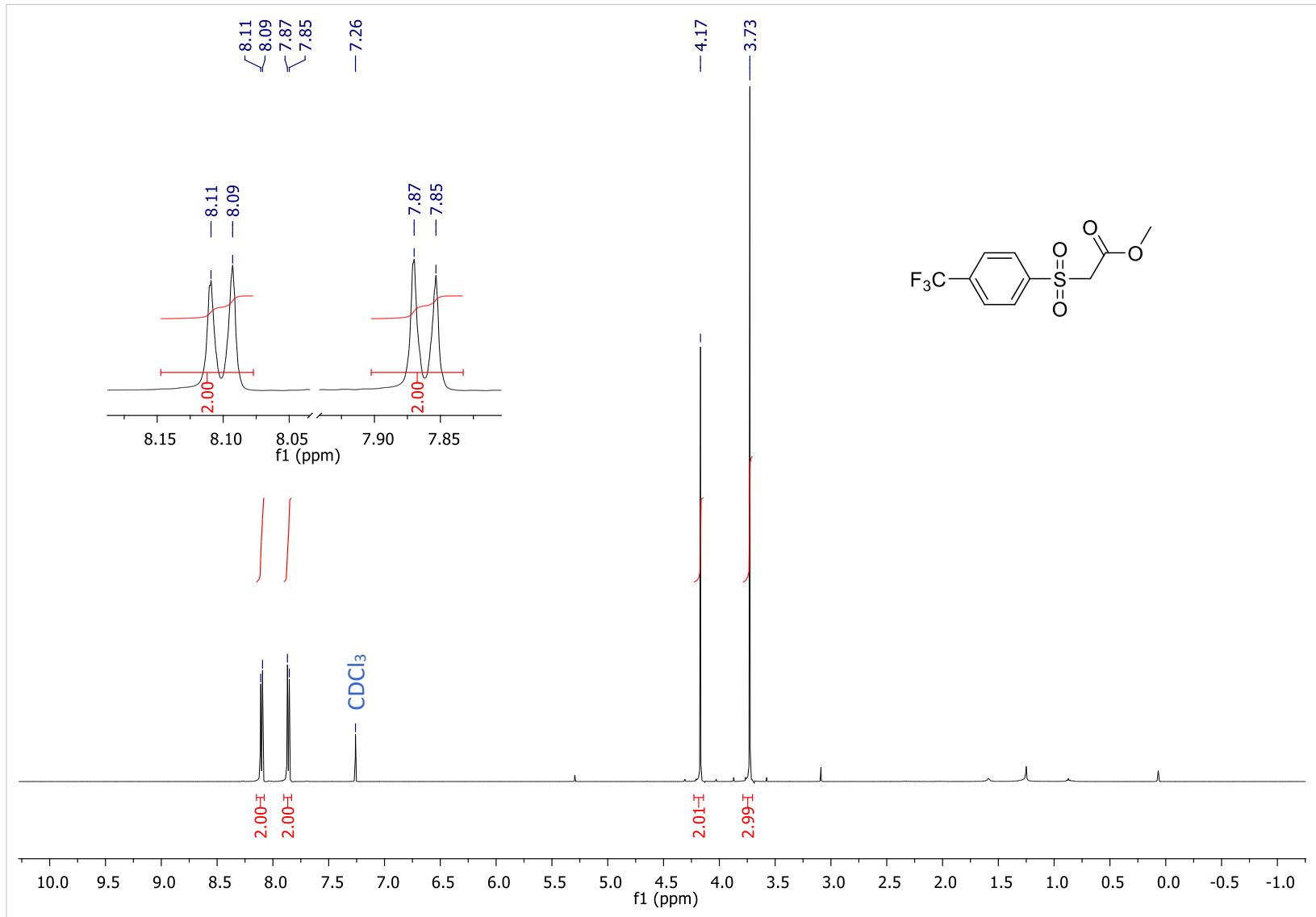

${ }^{13} \mathrm{C}\left\{{ }^{1} \mathrm{H}\right\} \mathrm{NMR}\left(126 \mathrm{MHz}, \mathrm{CDCl}_{3}\right)$

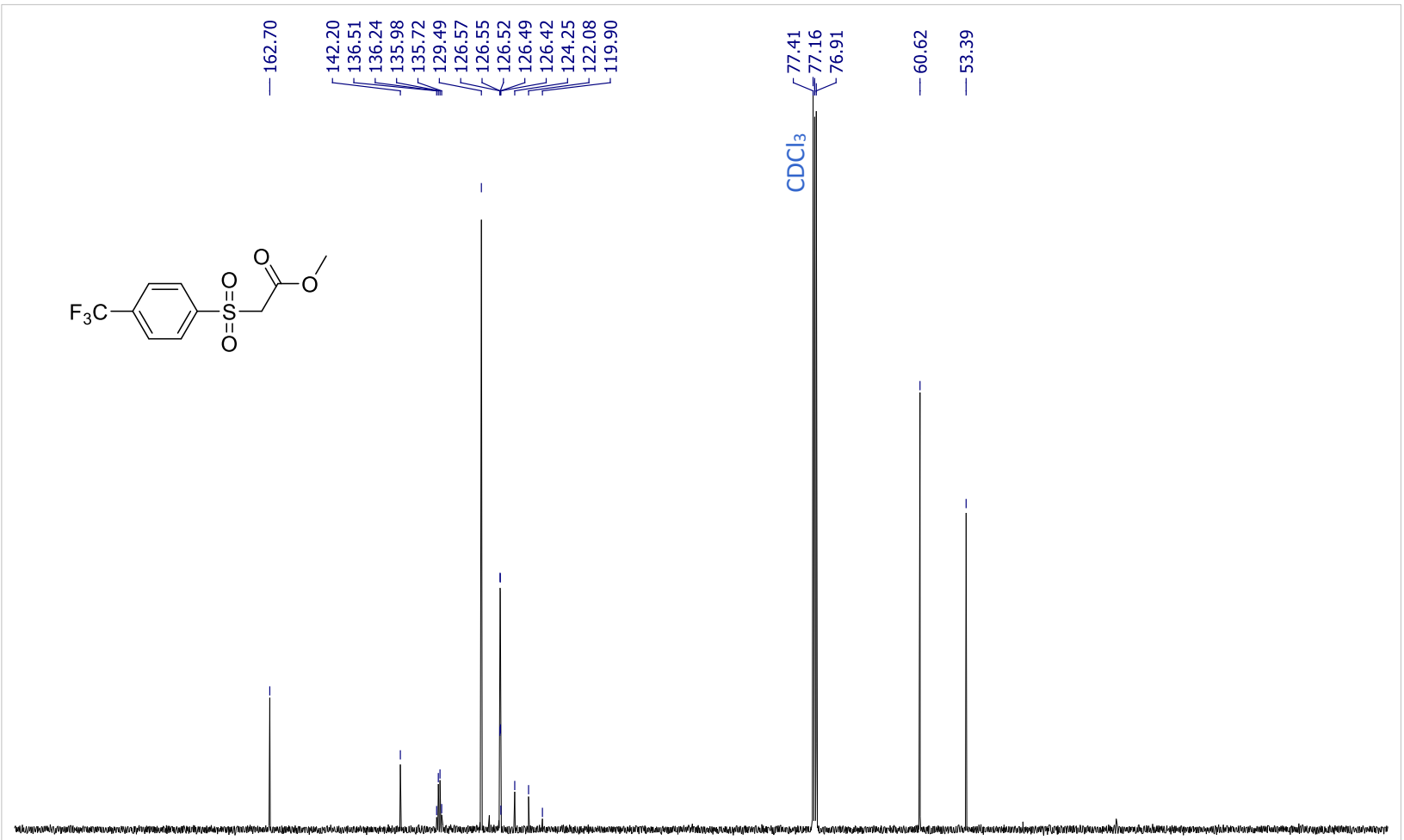

$\begin{array}{llllllllllllllllllllllll}200 & 190 & 180 & 170 & 160 & 150 & 140 & 130 & 120 & 110 & 100 & 90 & 80 & 70 & 60 & 50 & 40 & 30 & 20 & 10 & 0 & -10\end{array}$ 
${ }^{1} \mathrm{H} \mathrm{NMR}\left(500 \mathrm{MHz}, \mathrm{CDCl}_{3}\right)$

$3 q b$

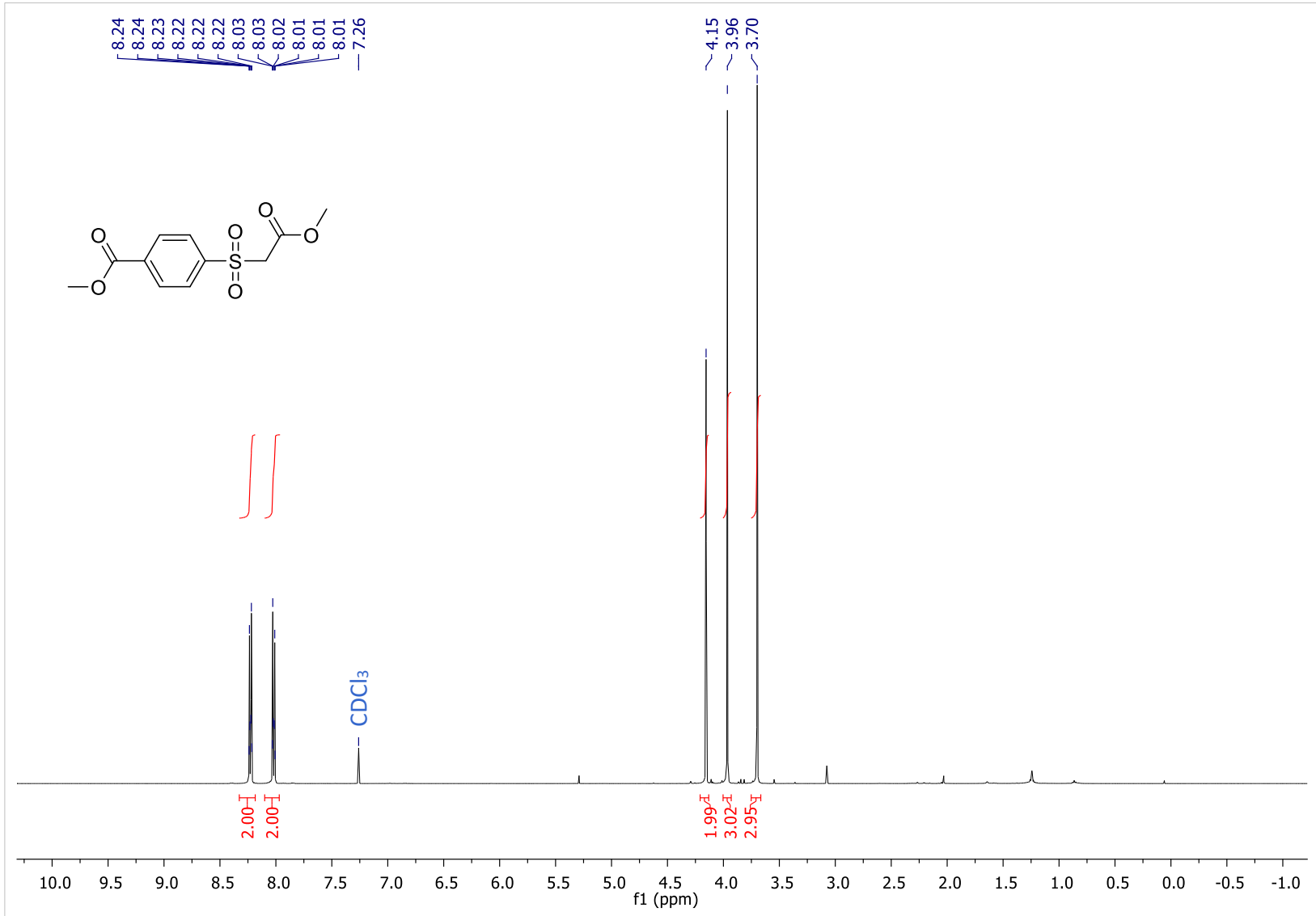

${ }^{13} \mathrm{C}\left\{{ }^{1} \mathrm{H}\right\}$ NMR $\left(126 \mathrm{MHz}, \mathrm{CDCl}_{3}\right)$

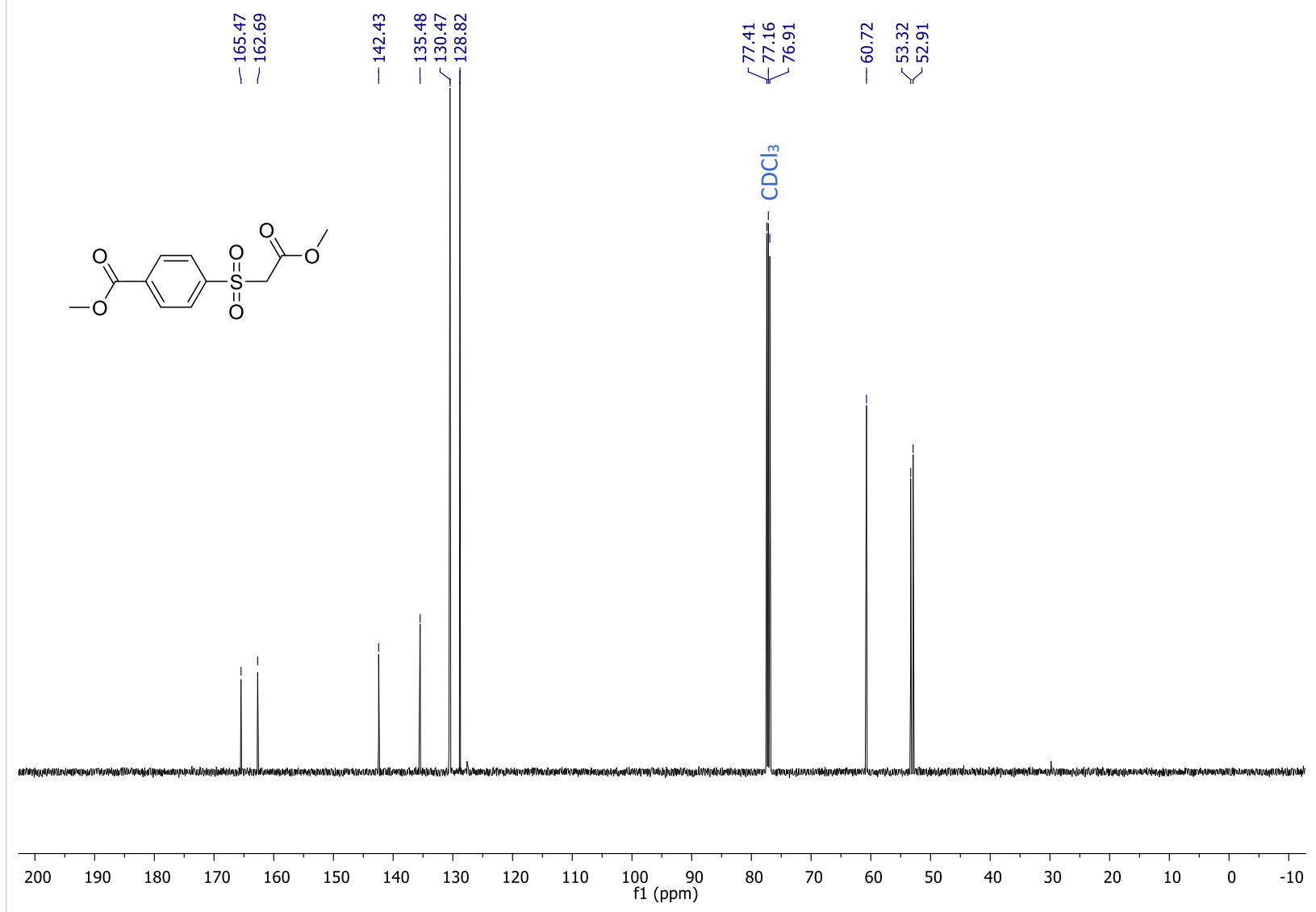


${ }^{1} \mathrm{H}$ NMR (500 MHz, DMSO- $d_{6}$ )

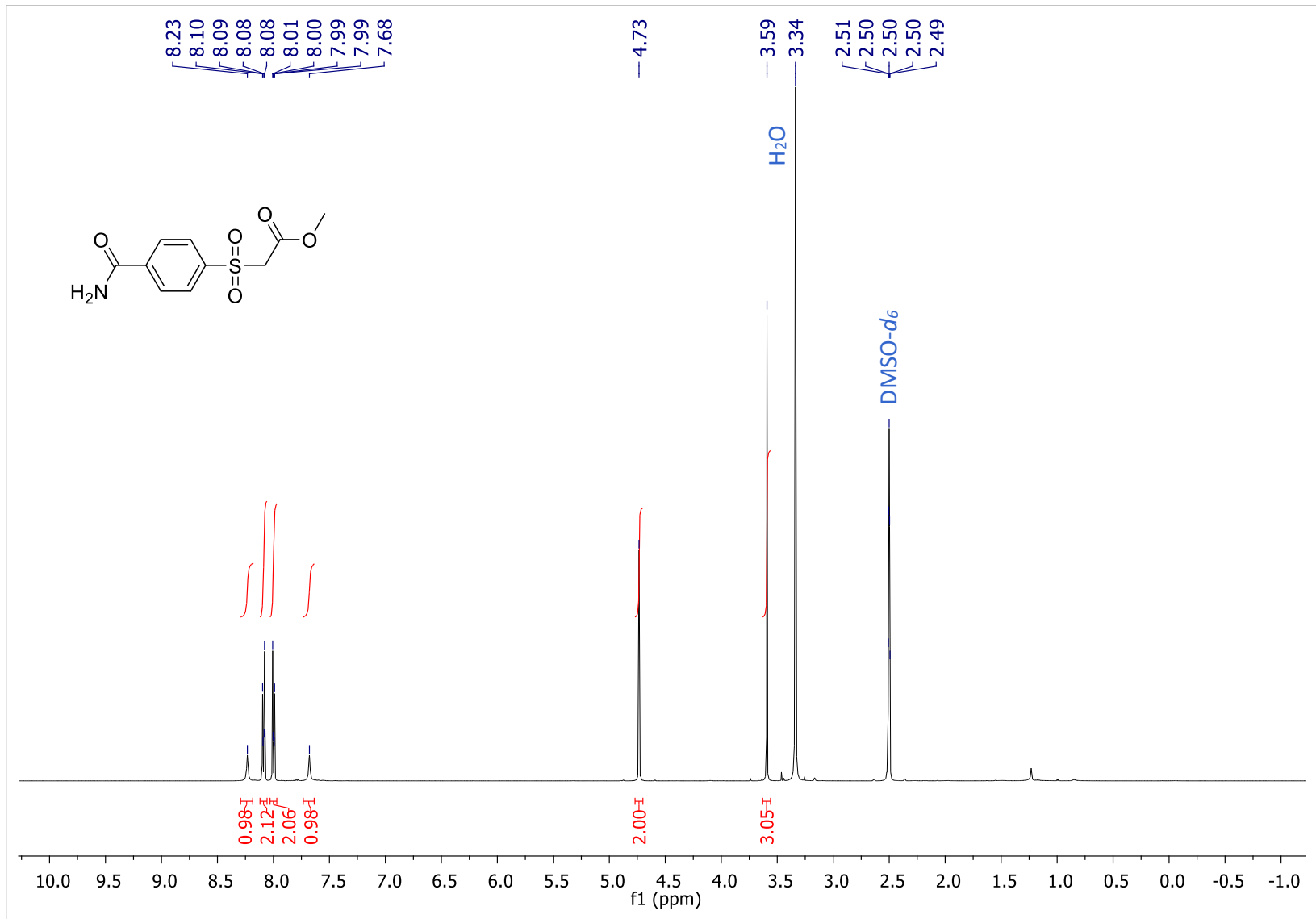

${ }^{13} \mathrm{C}\left\{{ }^{1} \mathrm{H}\right\}$ NMR (126 MHz, DMSO- $\left.d_{6}\right)$

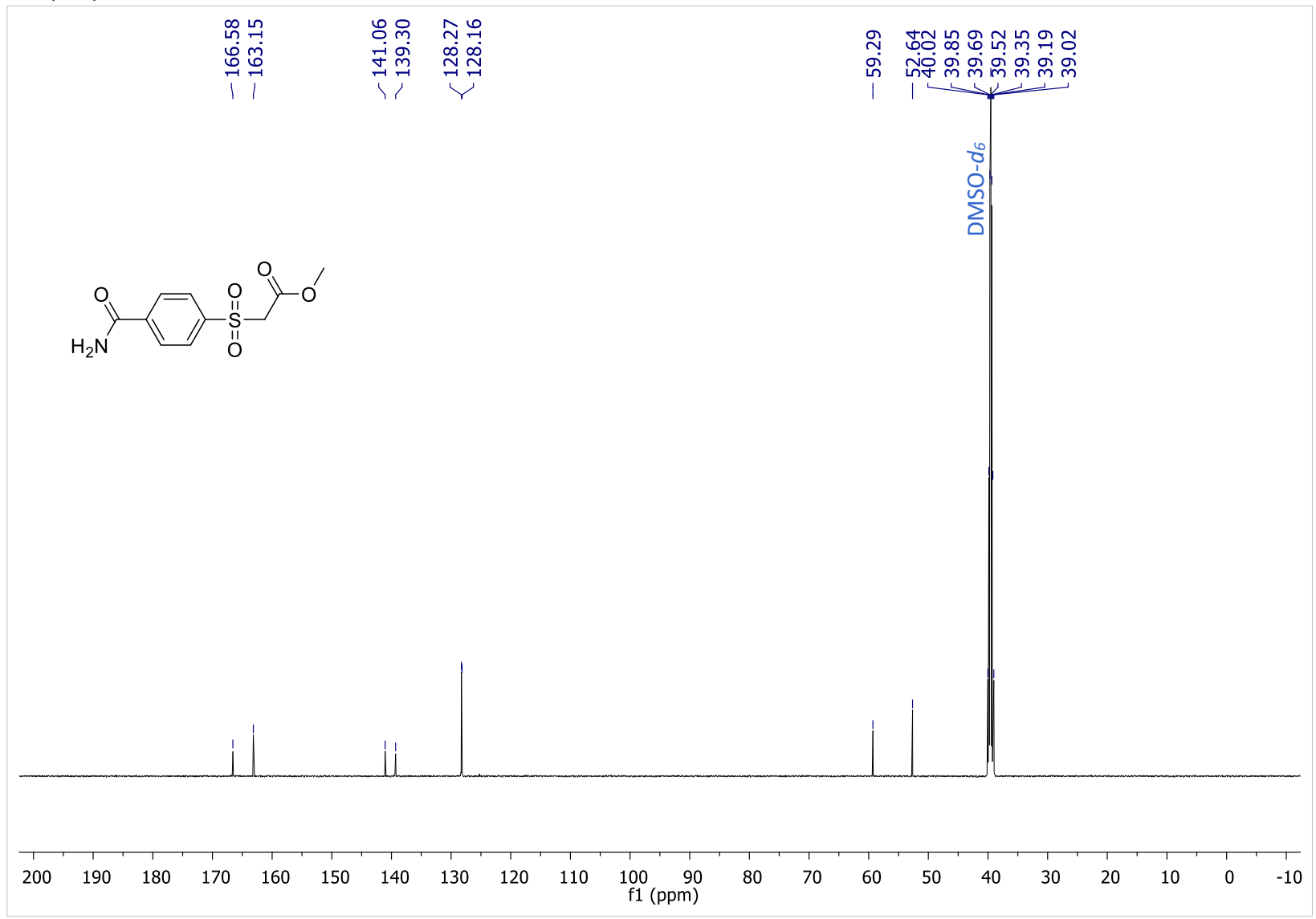


${ }^{1} \mathrm{H}$ NMR (500 MHz, $\mathrm{CDCl}_{3}$ )

3sb

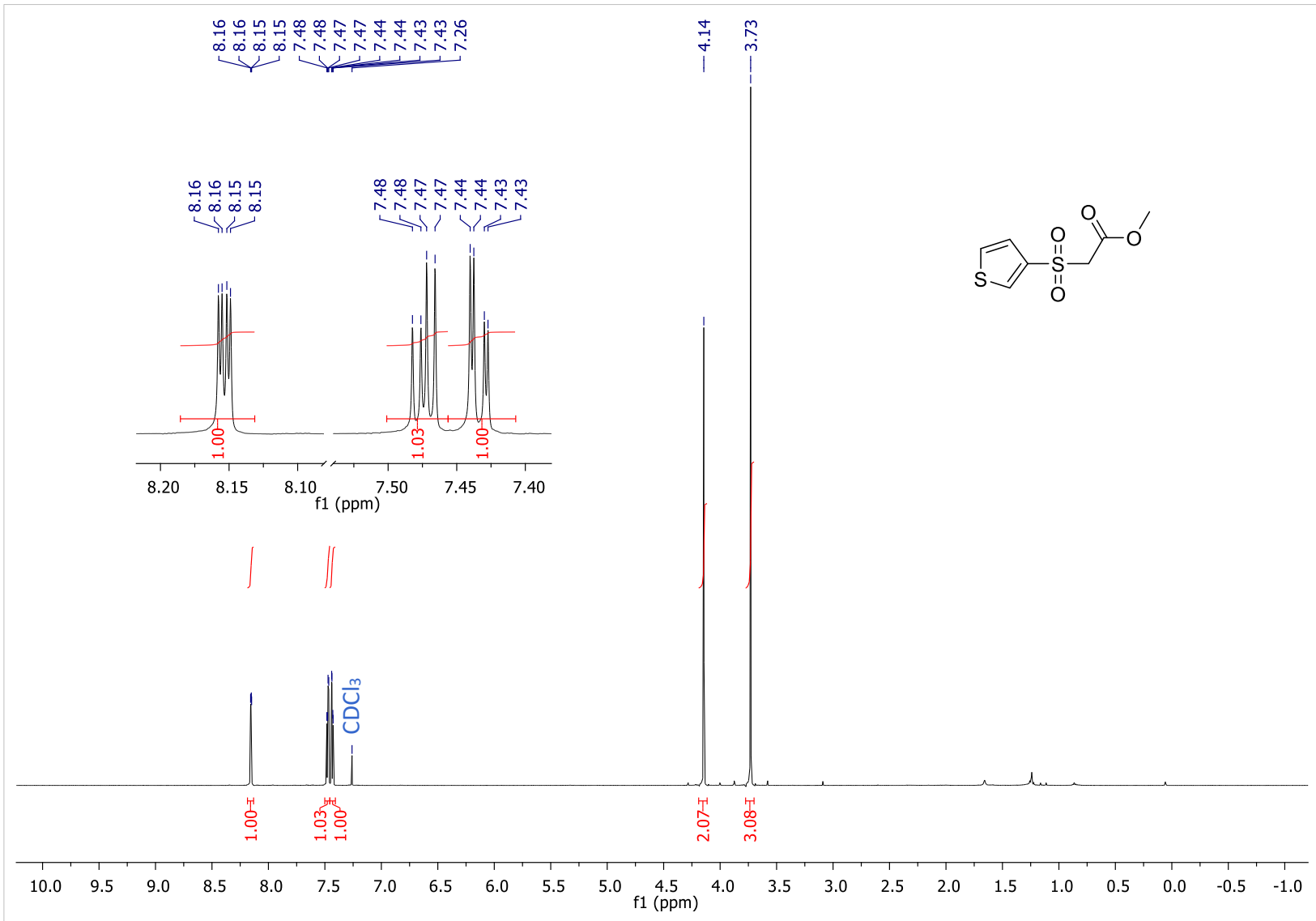

${ }^{13} \mathrm{C}\left\{{ }^{1} \mathrm{H}\right\}$ NMR (126 MHz, $\left.\mathrm{CDCl}_{3}\right)$

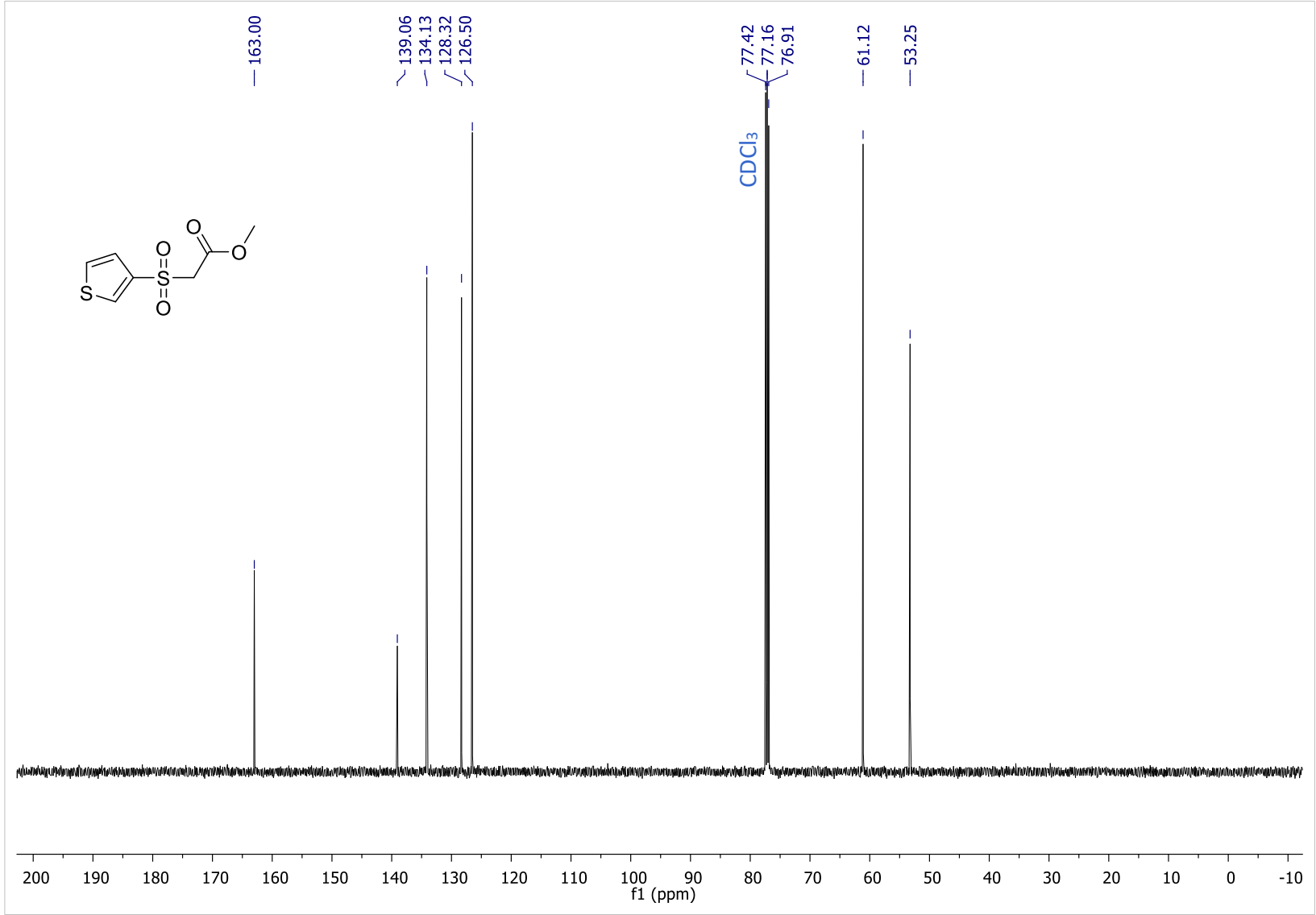


3tb

${ }^{1} \mathrm{H}$ NMR $\left(500 \mathrm{MHz}, \mathrm{CDCl}_{3}\right)$

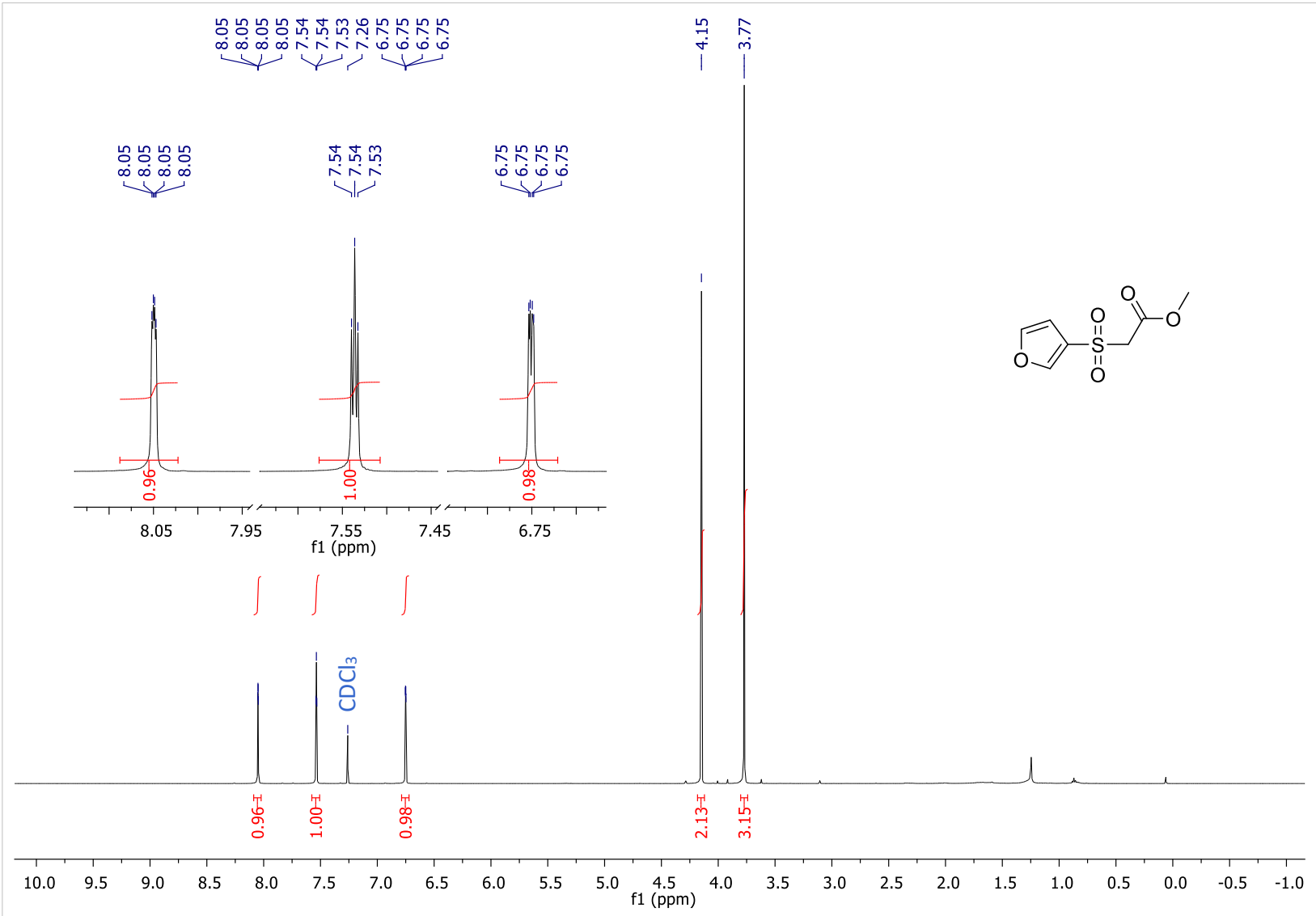

${ }^{13} \mathrm{C}\left\{{ }^{1} \mathrm{H}\right\}$ NMR $\left(126 \mathrm{MHz}, \mathrm{CDCl}_{3}\right)$

覆

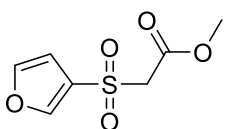

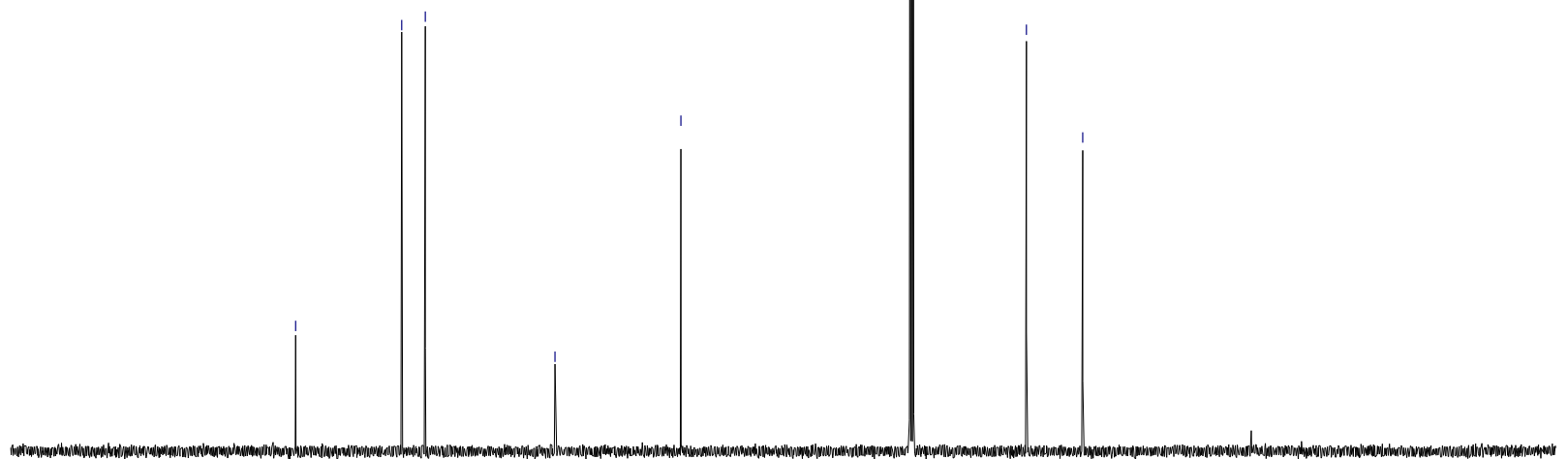

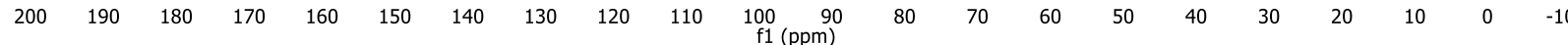


3ub

${ }^{1} \mathrm{H} \mathrm{NMR}\left(500 \mathrm{MHz}, \mathrm{CDCl}_{3}\right)$

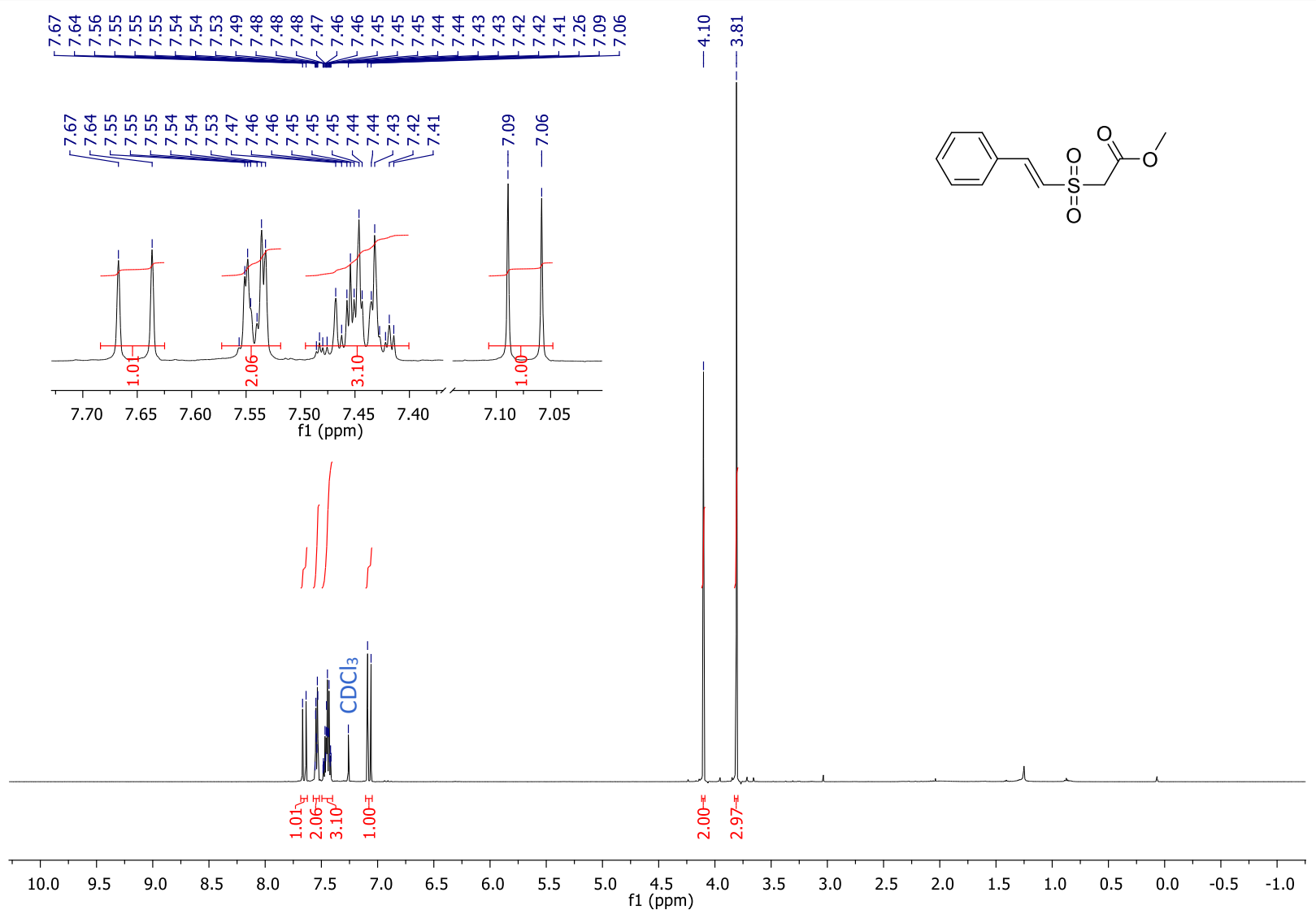

${ }^{13} \mathrm{C}\left\{{ }^{1} \mathrm{H}\right\}$ NMR (126 MHz, $\left.\mathrm{CDCl}_{3}\right)$

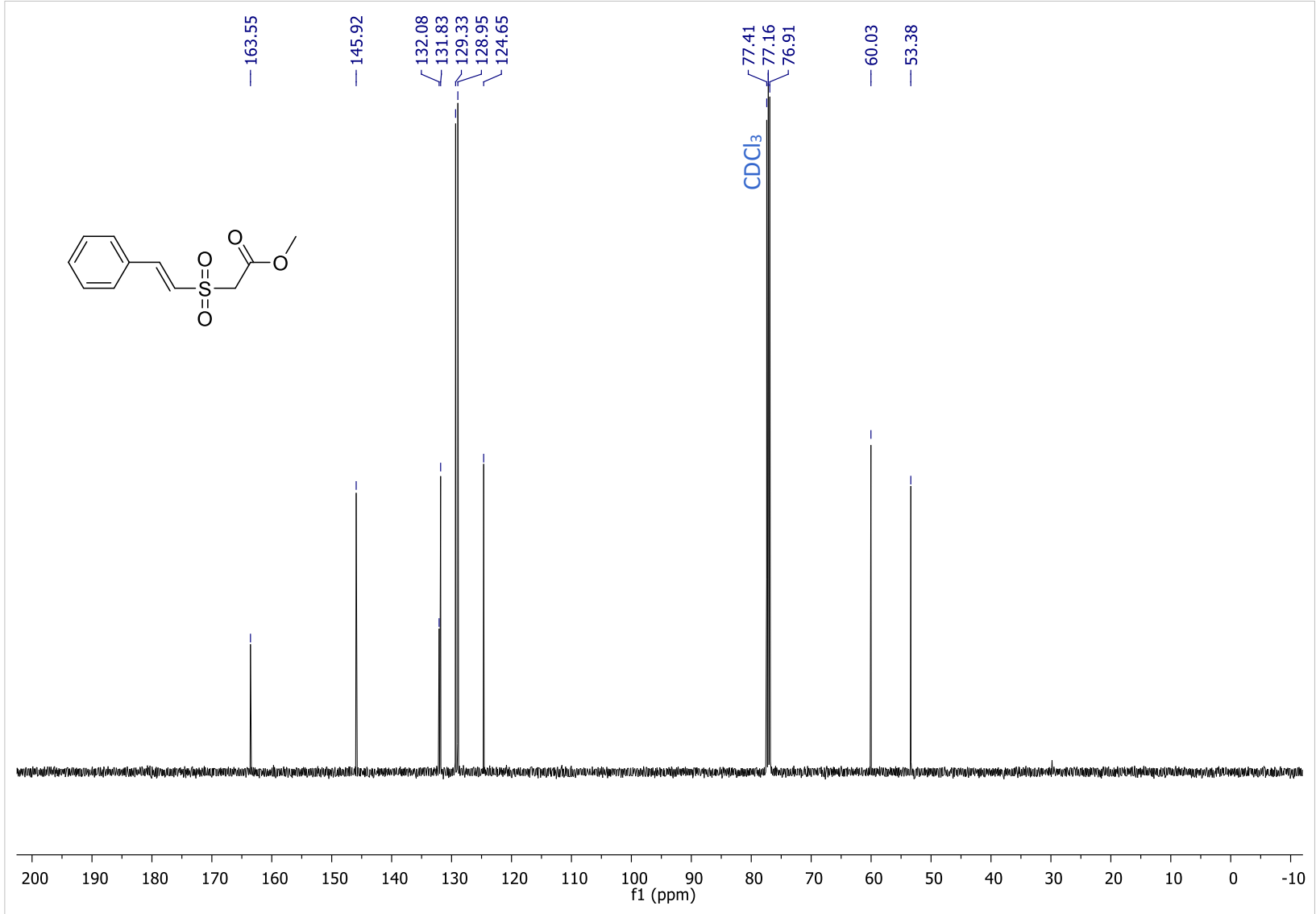




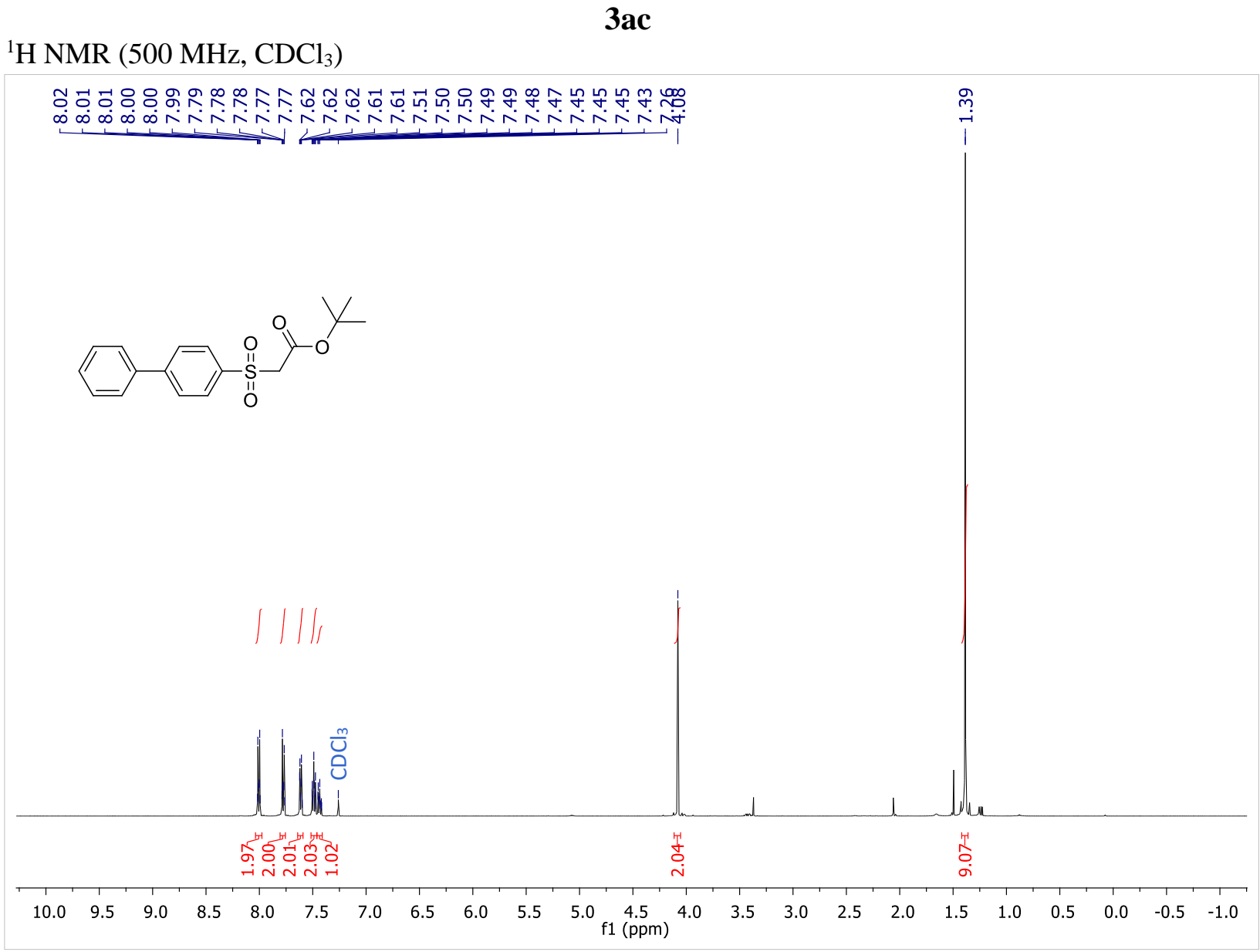

${ }^{13} \mathrm{C}\left\{{ }^{1} \mathrm{H}\right\} \mathrm{NMR}\left(126 \mathrm{MHz}, \mathrm{CDCl}_{3}\right)$

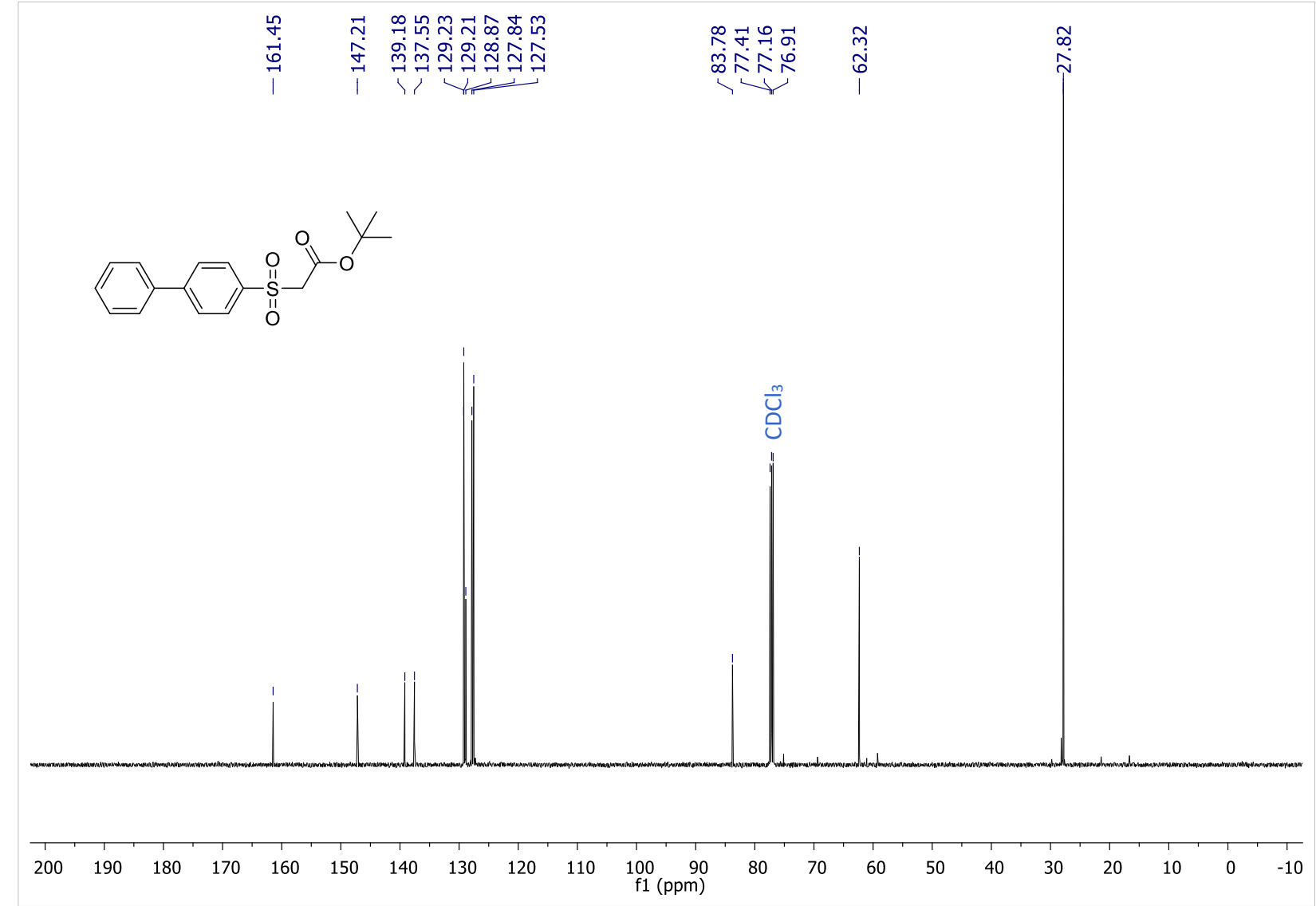


3ad

${ }^{1} \mathrm{H} \mathrm{NMR}\left(500 \mathrm{MHz}, \mathrm{CDCl}_{3}\right)$

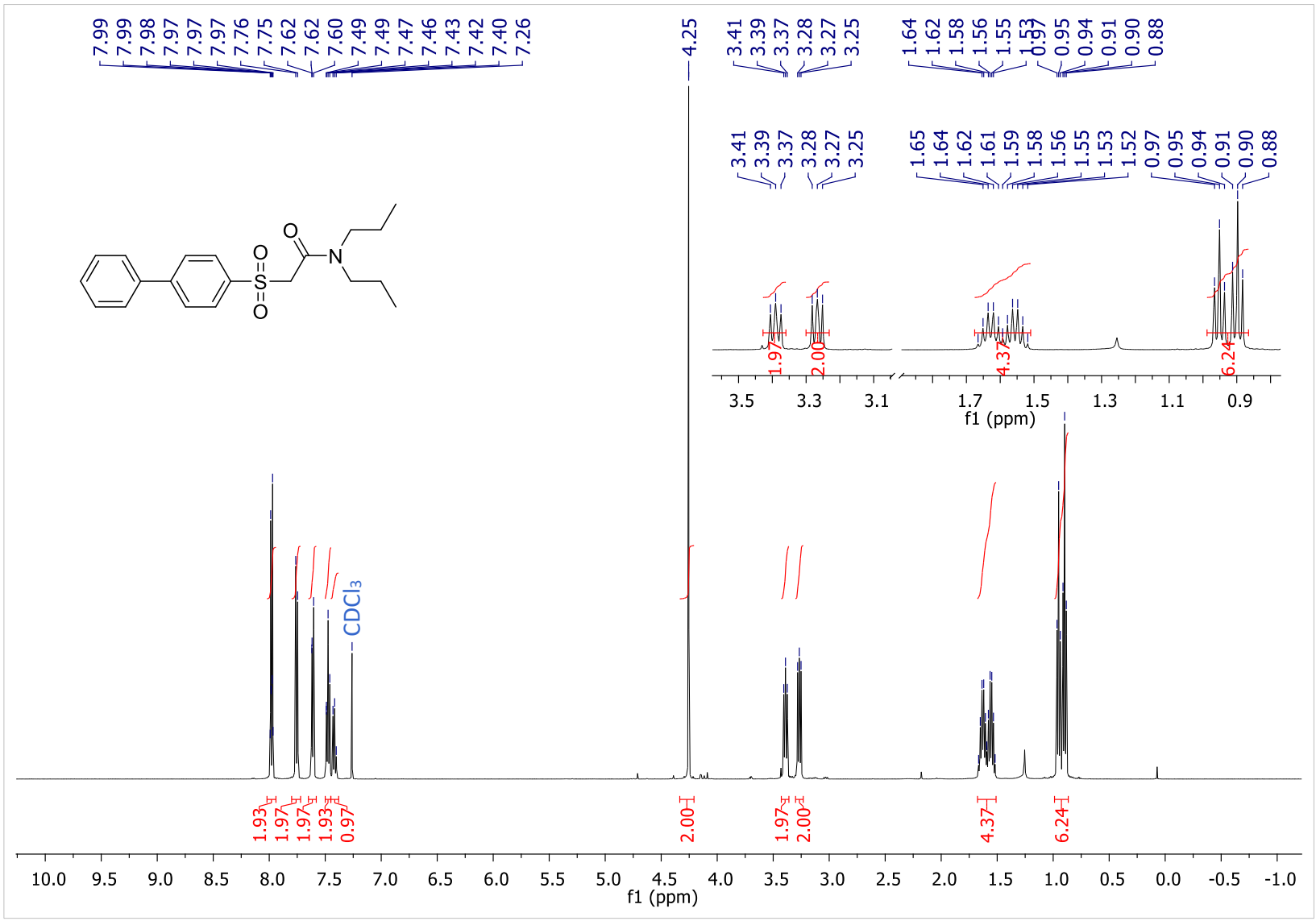

${ }^{13} \mathrm{C}\left\{{ }^{1} \mathrm{H}\right\}$ NMR $\left(126 \mathrm{MHz}, \mathrm{CDCl}_{3}\right)$

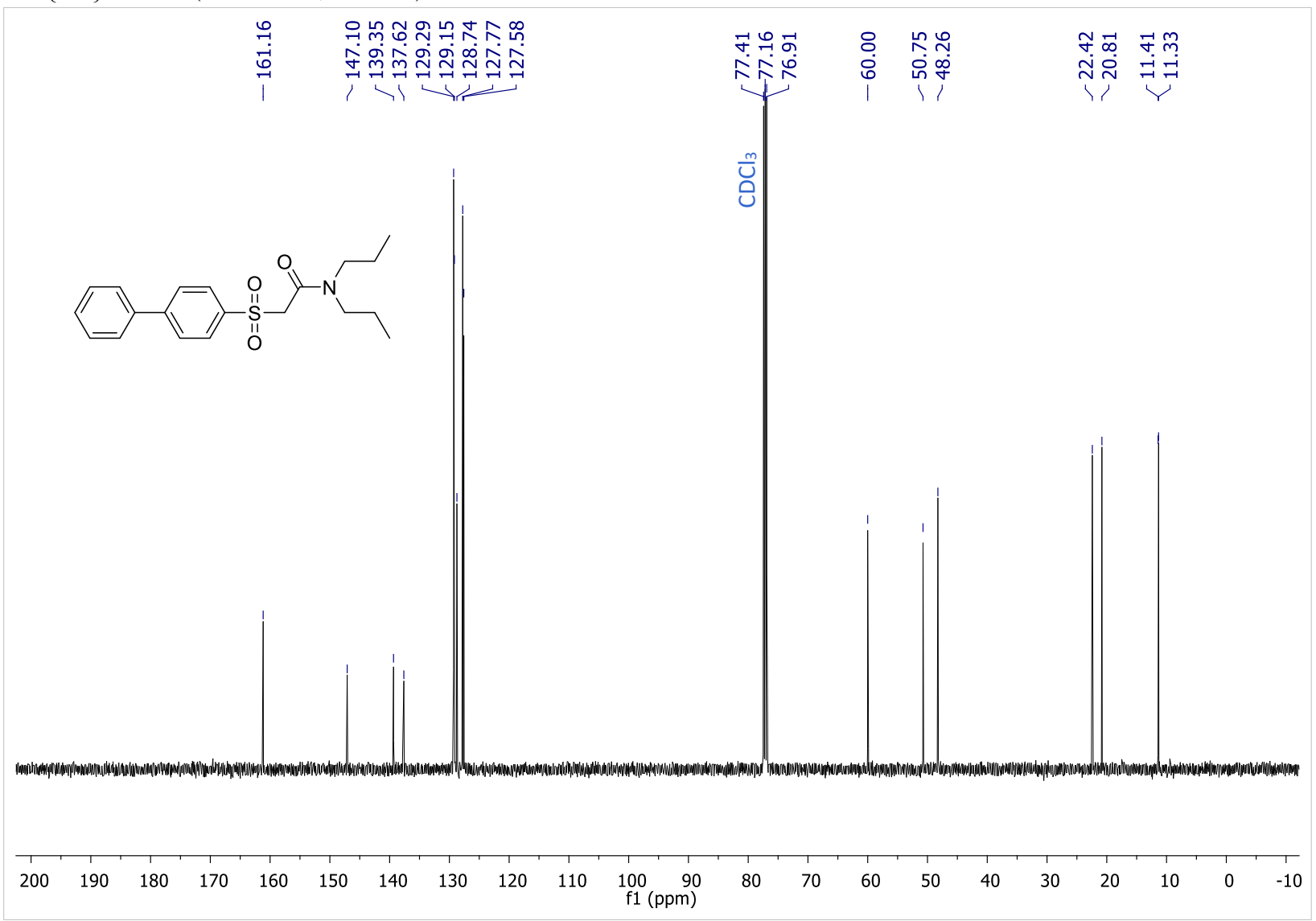


${ }^{1} \mathrm{H}$ NMR $\left(500 \mathrm{MHz}, \mathrm{CDCl}_{3}\right)$

3ae

ঞ

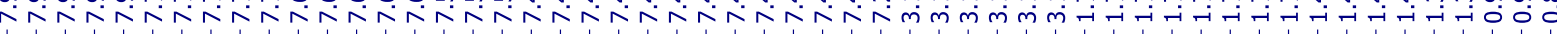

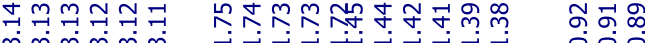
min<smiles>CCCCS(=O)(=O)c1ccc(-c2ccccc2)cc1</smiles>

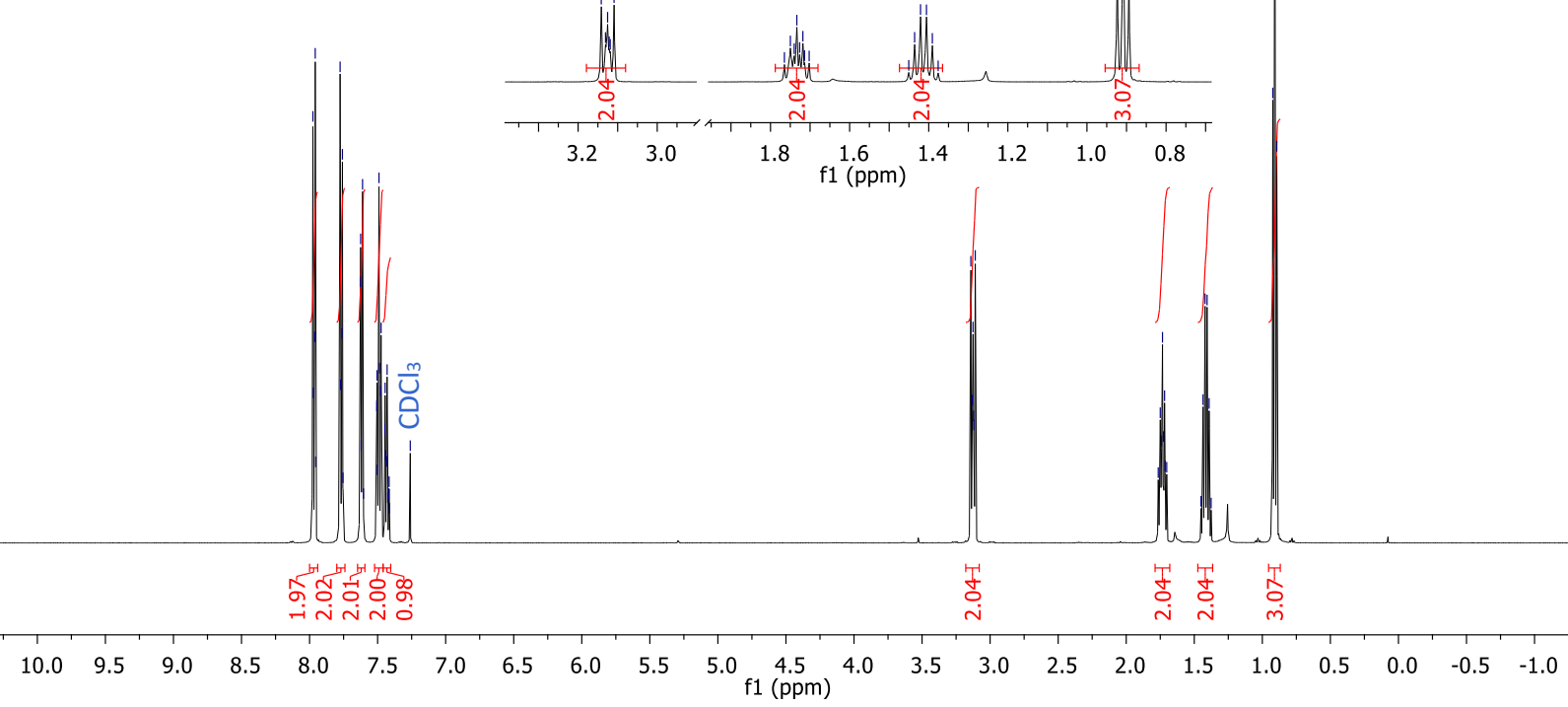

${ }^{13} \mathrm{C}\left\{{ }^{1} \mathrm{H}\right\}$ NMR (126 MHz, $\left.\mathrm{CDCl}_{3}\right)$

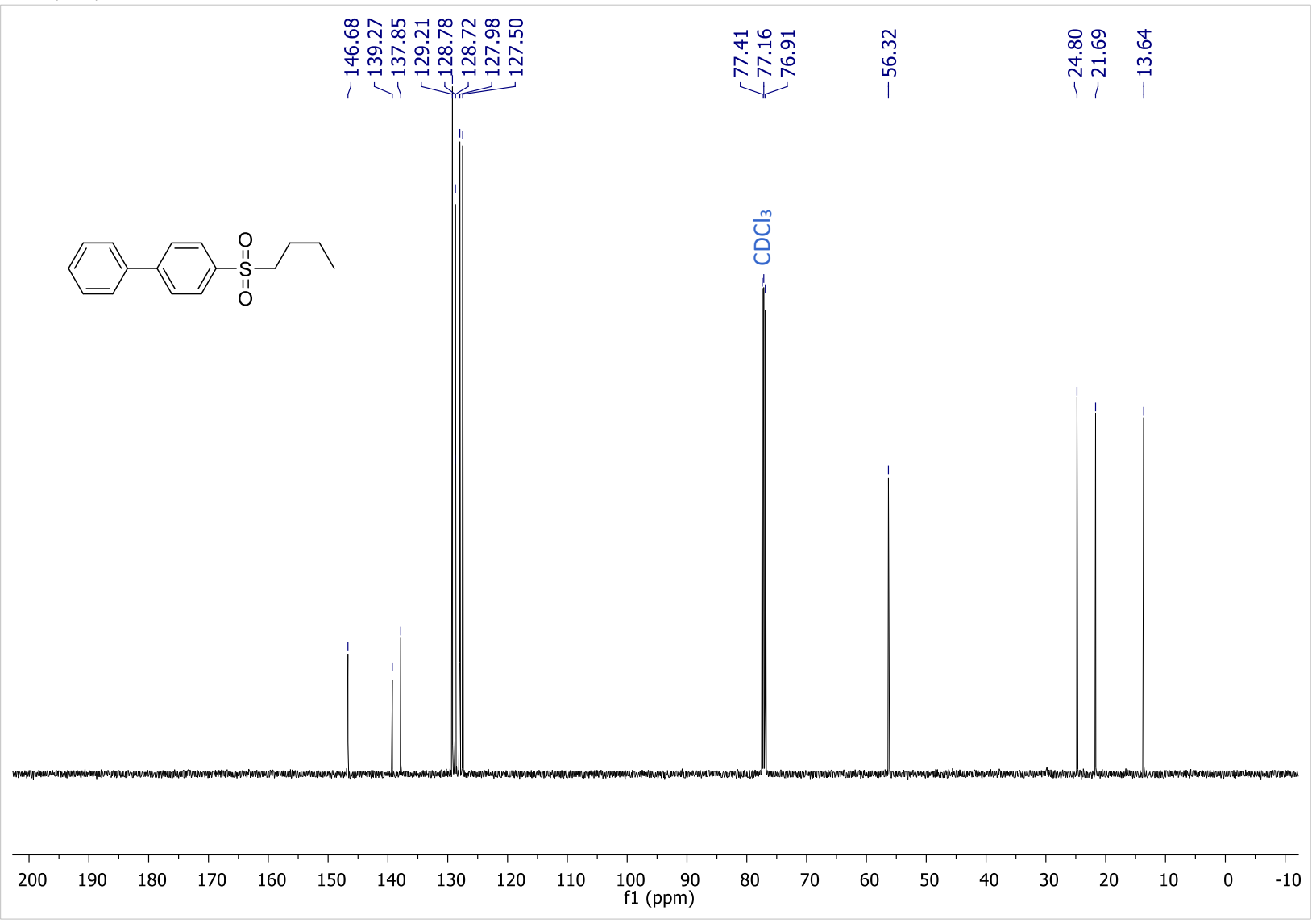




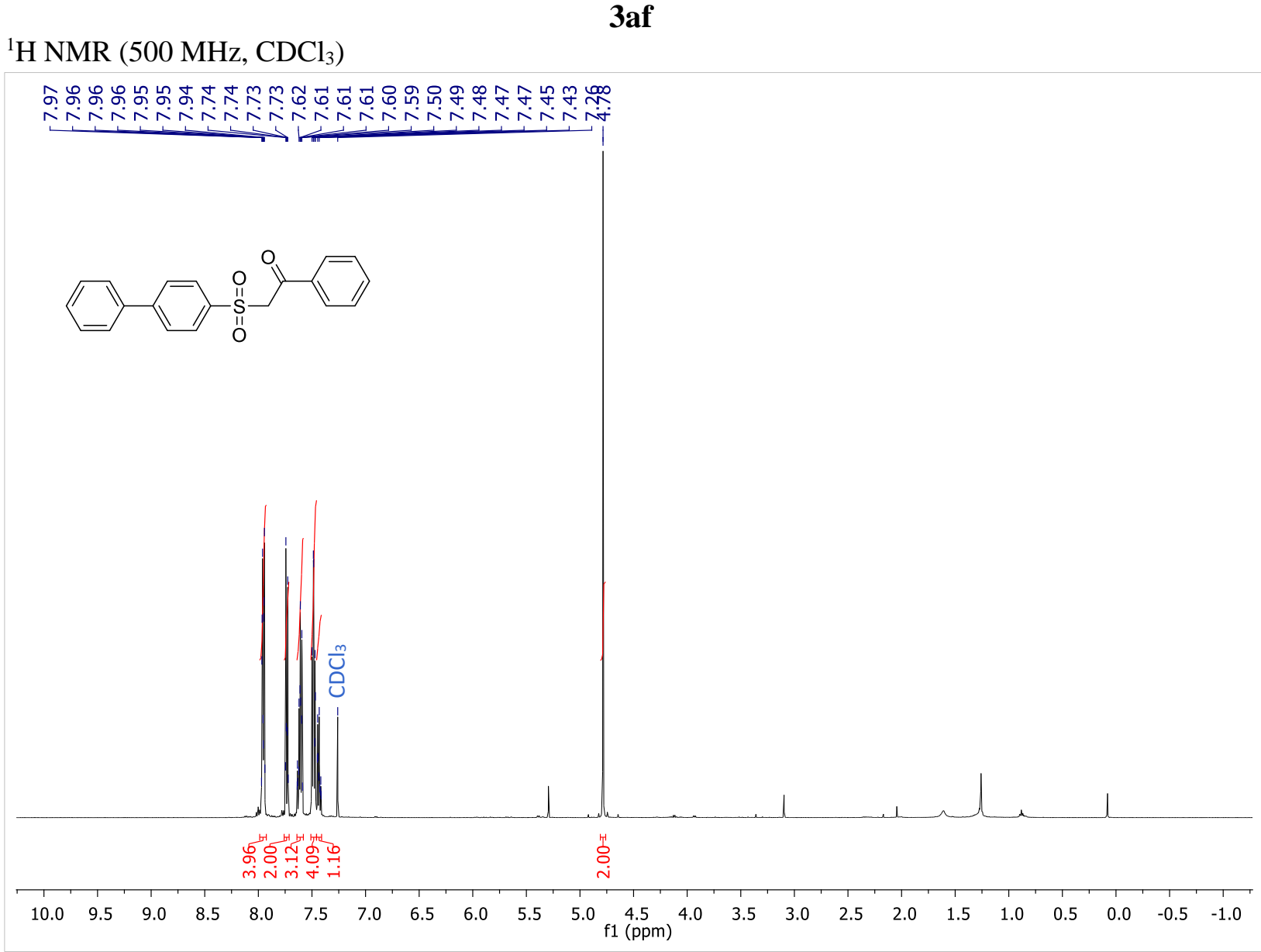

${ }^{13} \mathrm{C}\left\{{ }^{1} \mathrm{H}\right\}$ NMR (126 MHz, $\left.\mathrm{CDCl}_{3}\right)$

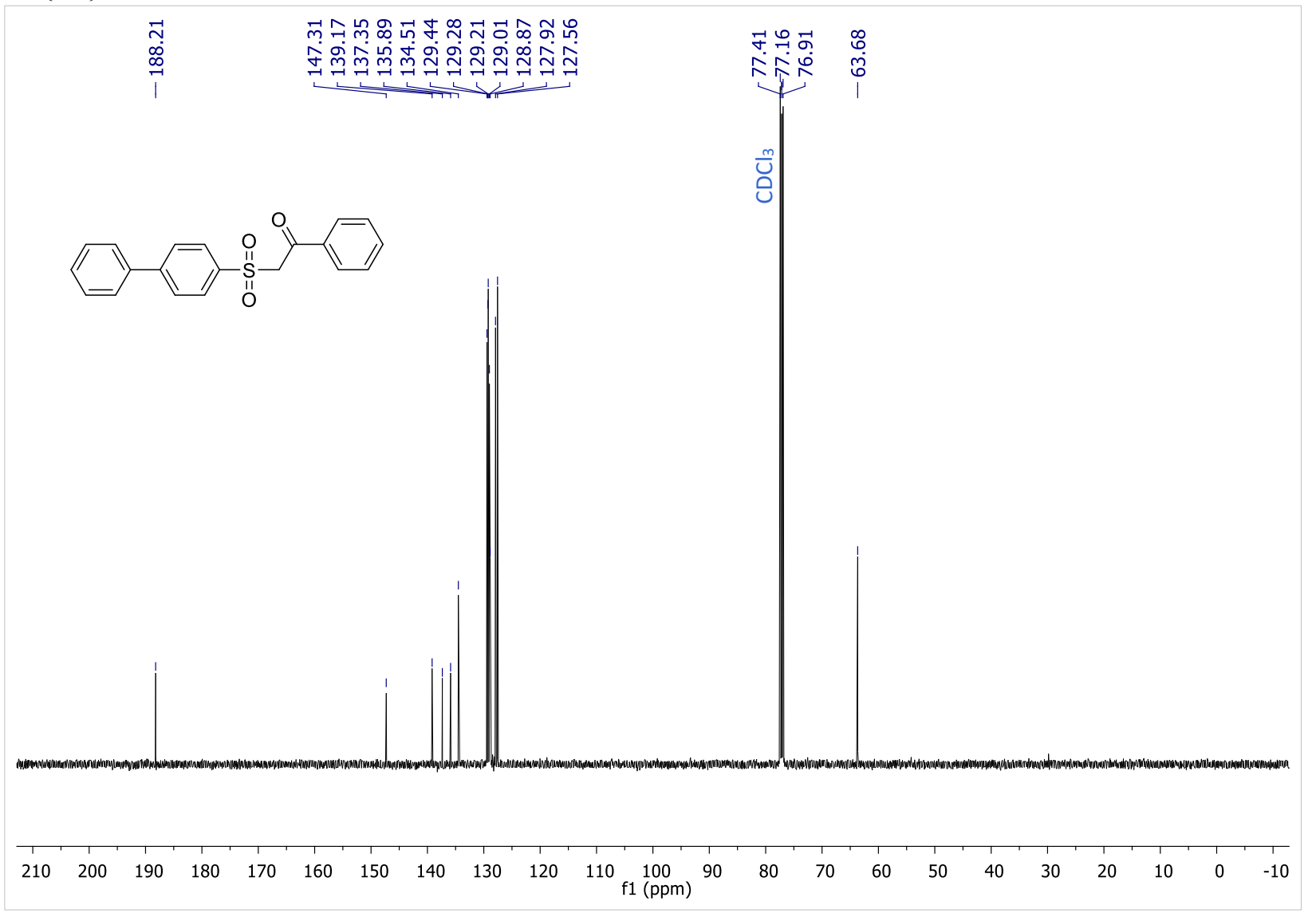


${ }^{1} \mathrm{H} \mathrm{NMR}\left(500 \mathrm{MHz}, \mathrm{CDCl}_{3}\right)$

3ag

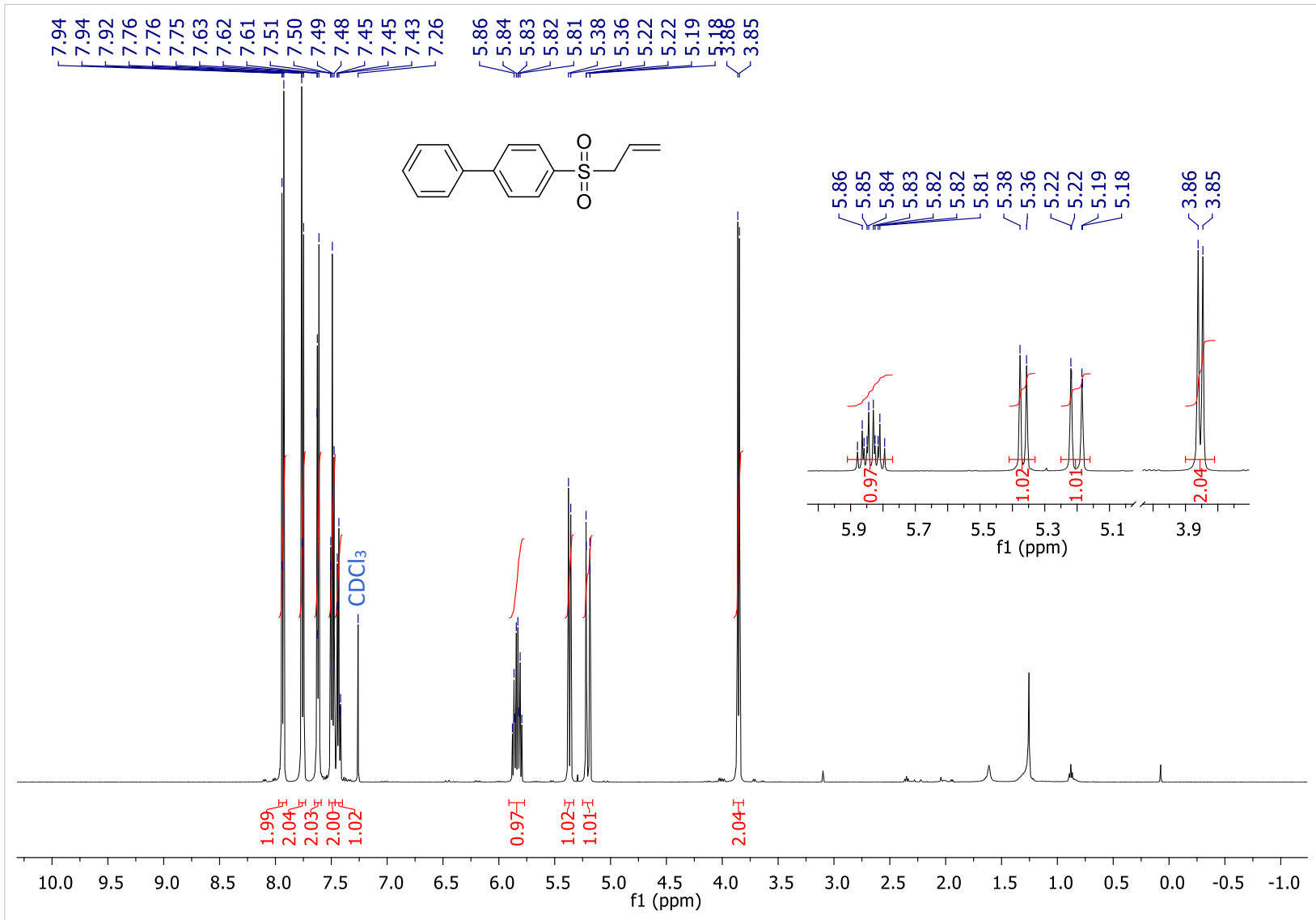

${ }^{13} \mathrm{C}\left\{{ }^{1} \mathrm{H}\right\}$ NMR $\left(126 \mathrm{MHz}, \mathrm{CDCl}_{3}\right)$

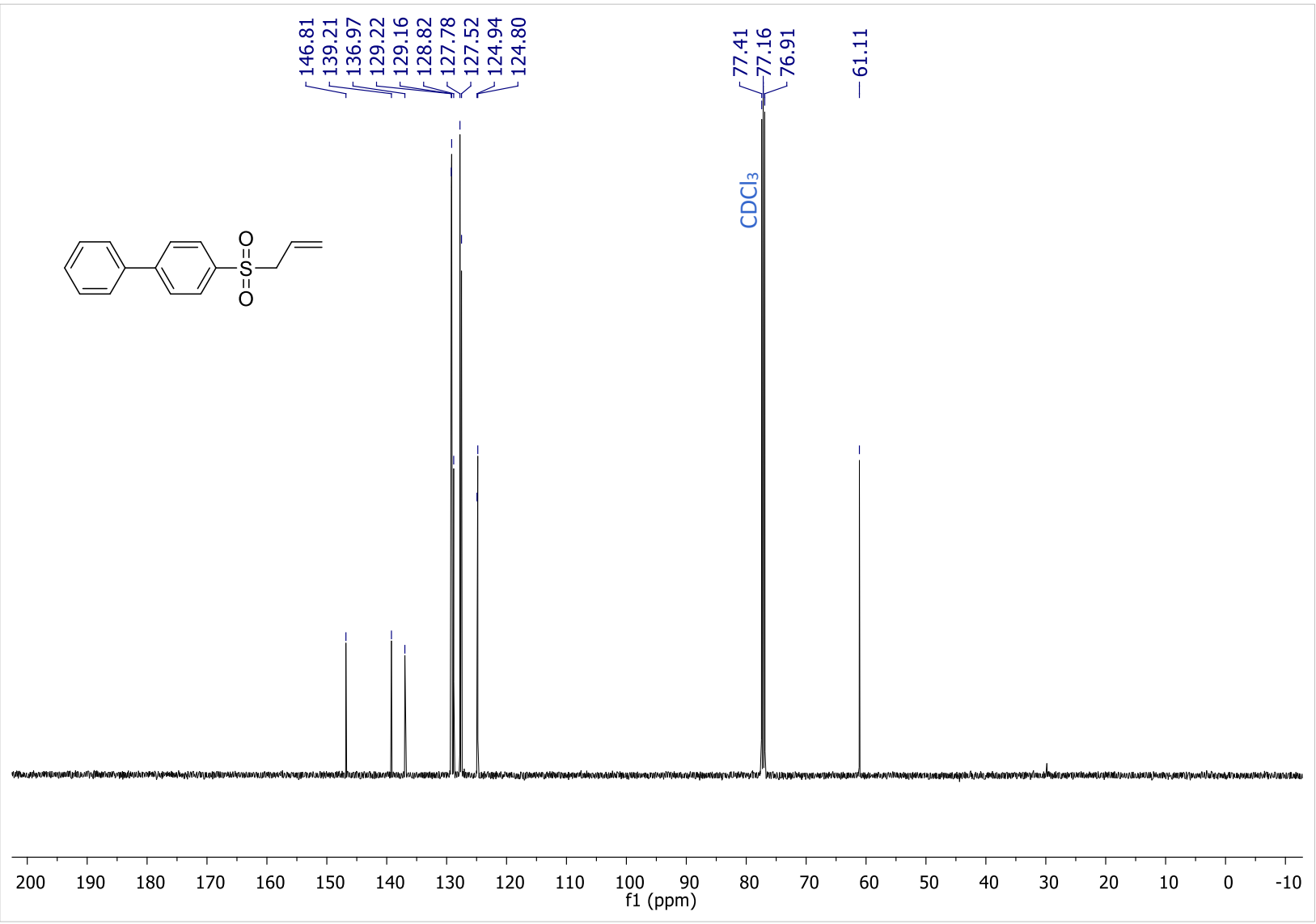


${ }^{1} \mathrm{H}$ NMR (500 MHz, $\mathrm{CDCl}_{3}$ )

3ah

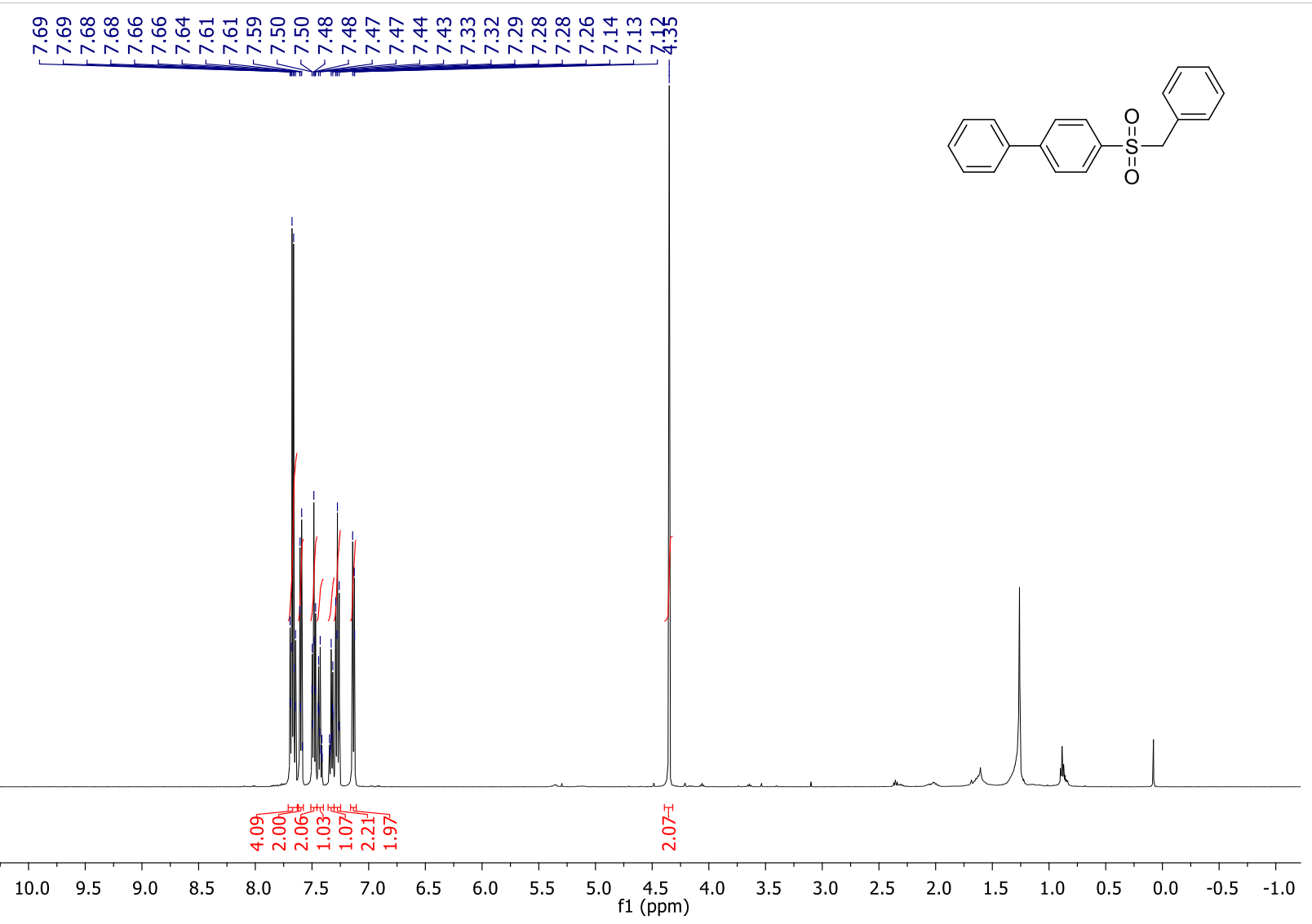

${ }^{13} \mathrm{C}\left\{{ }^{1} \mathrm{H}\right\}$ NMR (126 MHz, $\left.\mathrm{CDCl}_{3}\right)$

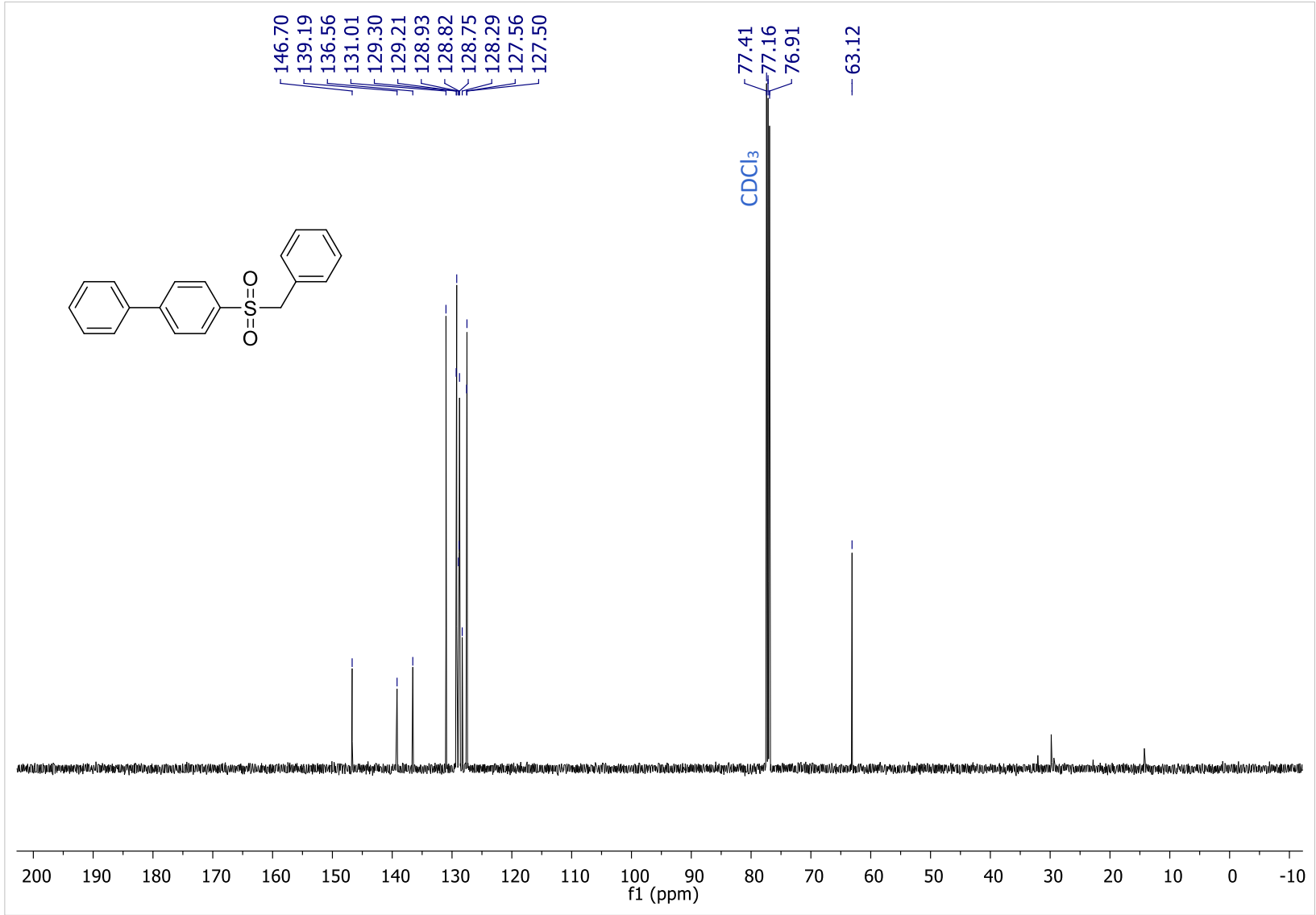


${ }^{1} \mathrm{H} \mathrm{NMR}\left(500 \mathrm{MHz}, \mathrm{CDCl}_{3}\right)$

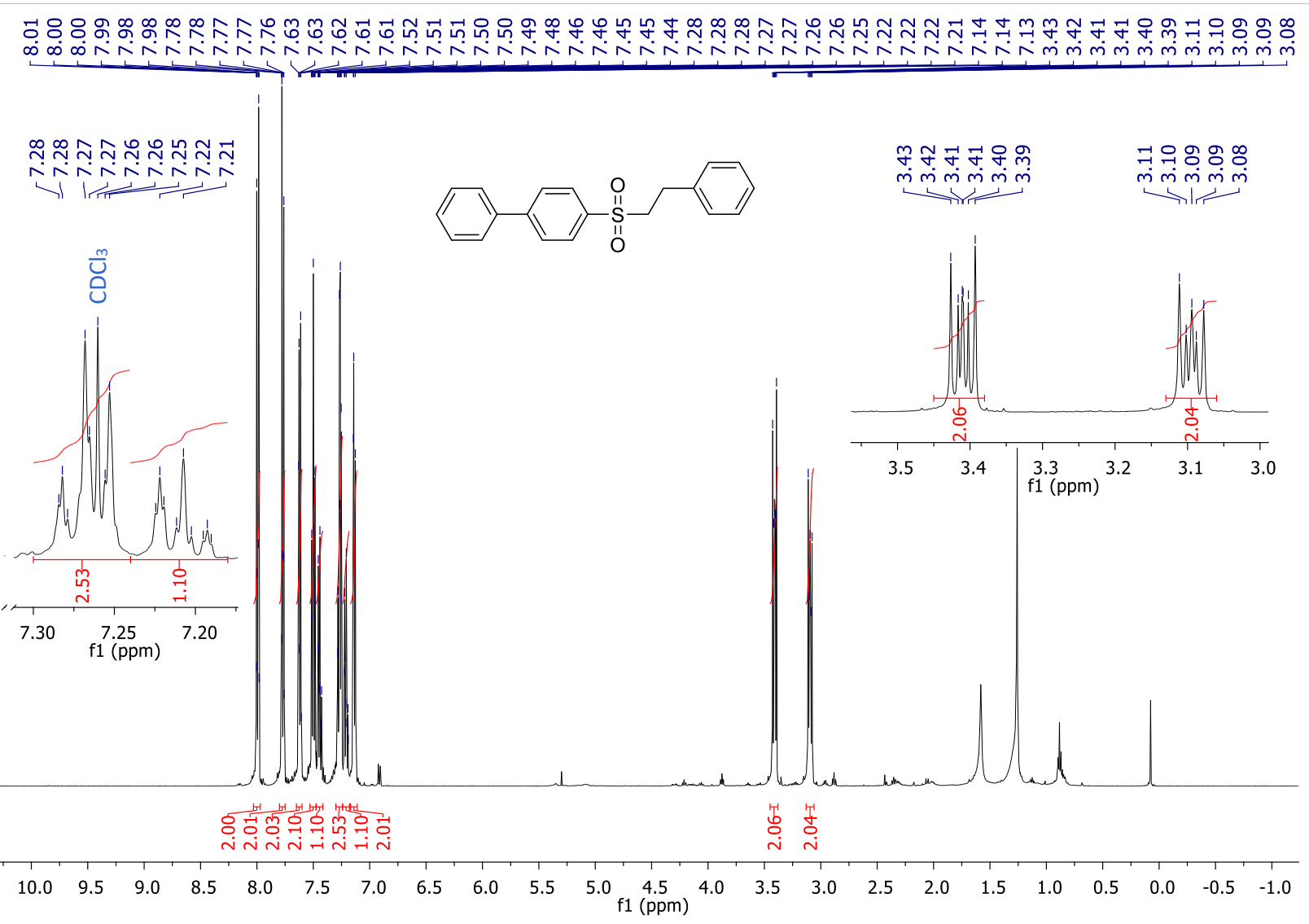

${ }^{13} \mathrm{C}\left\{{ }^{1} \mathrm{H}\right\}$ NMR $\left(126 \mathrm{MHz}, \mathrm{CDCl}_{3}\right)$

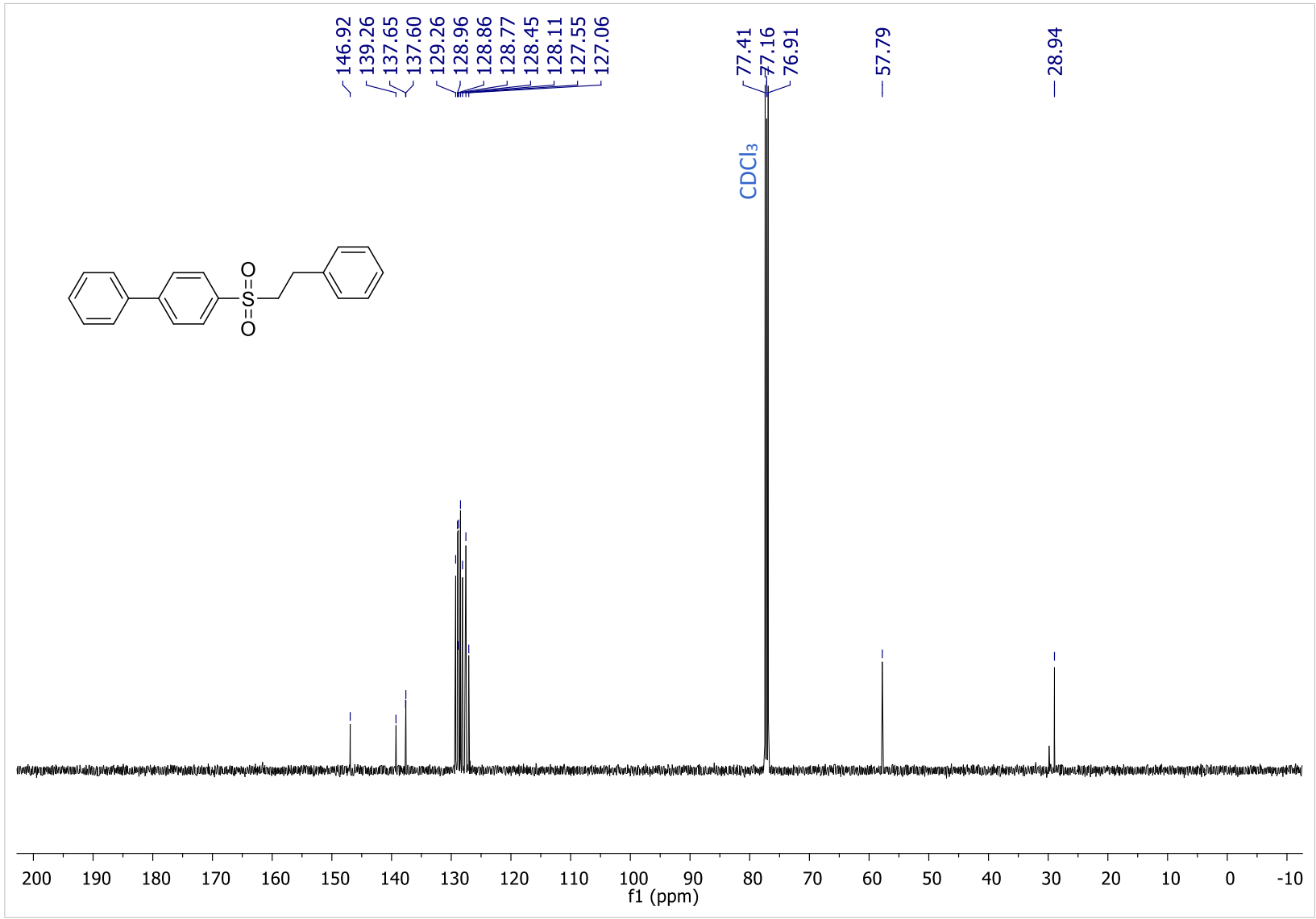


${ }^{1} \mathrm{H} \mathrm{NMR}\left(500 \mathrm{MHz}, \mathrm{CDCl}_{3}\right)$

3aj

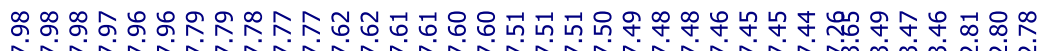

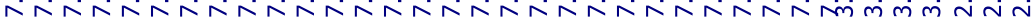

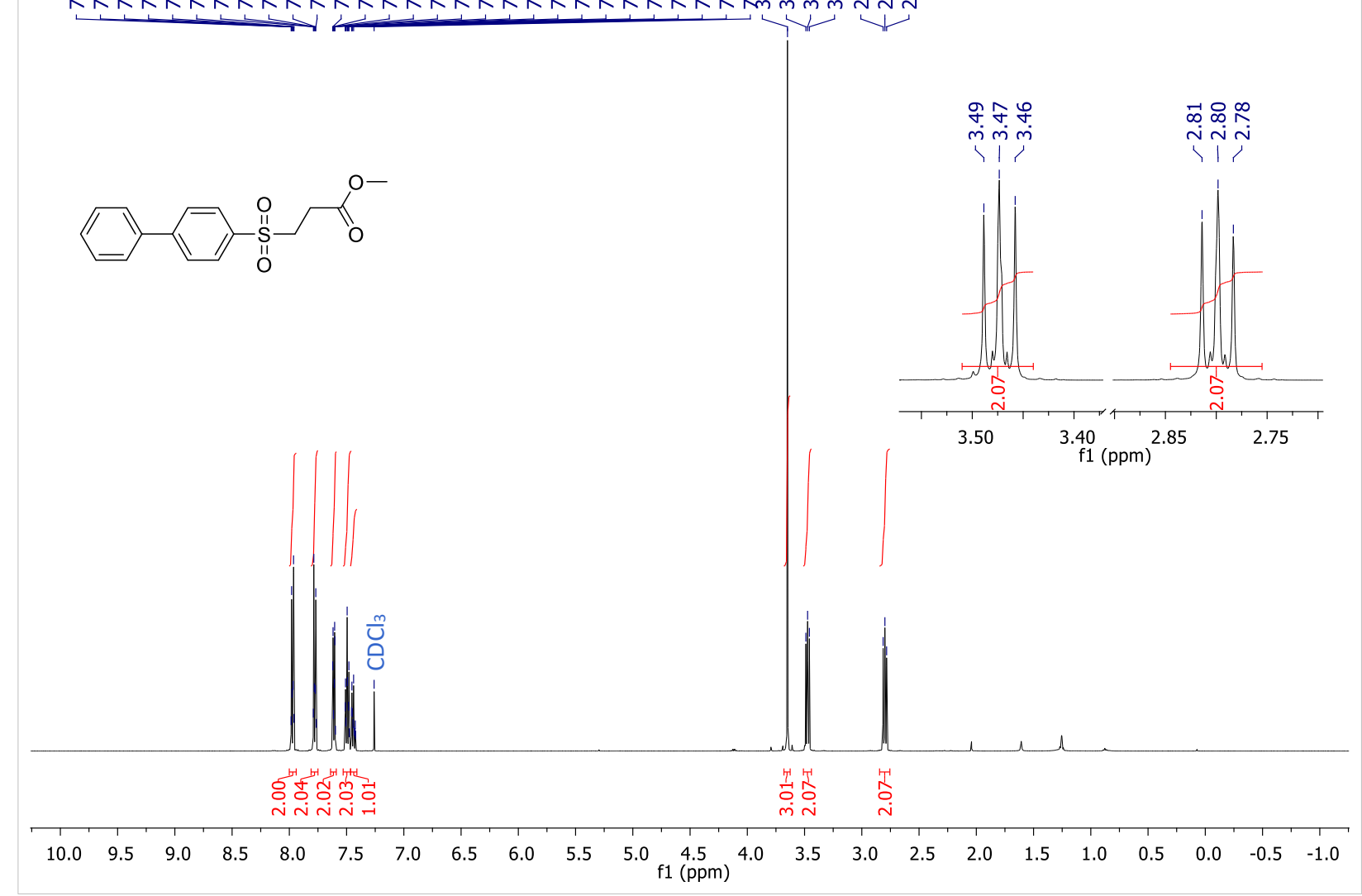

${ }^{13} \mathrm{C}\left\{{ }^{1} \mathrm{H}\right\}$ NMR $\left(126 \mathrm{MHz}, \mathrm{CDCl}_{3}\right)$

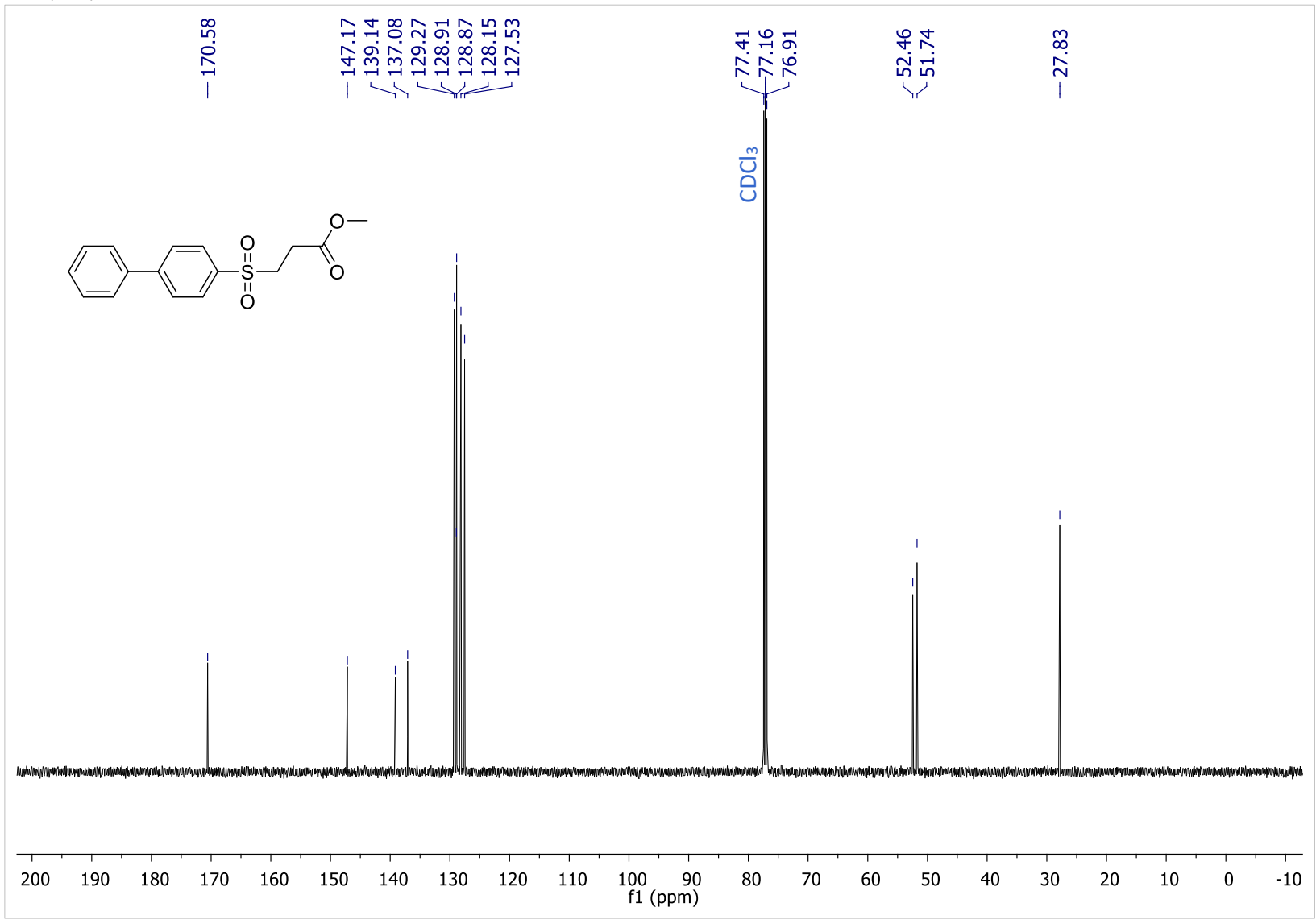


${ }^{1} \mathrm{H}$ NMR (500 MHz, $\mathrm{CDCl}_{3}$ )

3ak

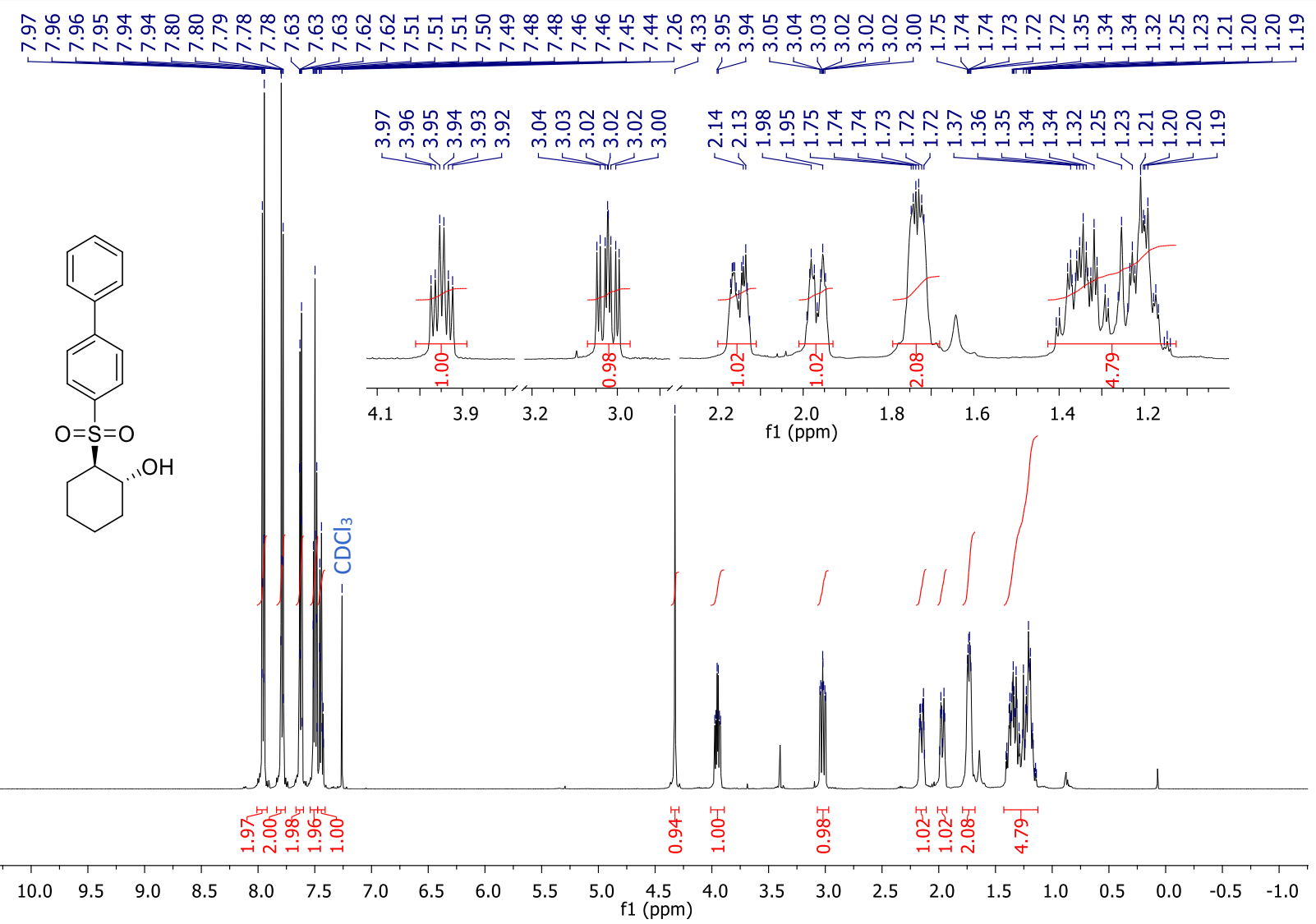

${ }^{13} \mathrm{C}\left\{{ }^{1} \mathrm{H}\right\}$ NMR (126 MHz, $\left.\mathrm{CDCl}_{3}\right)$

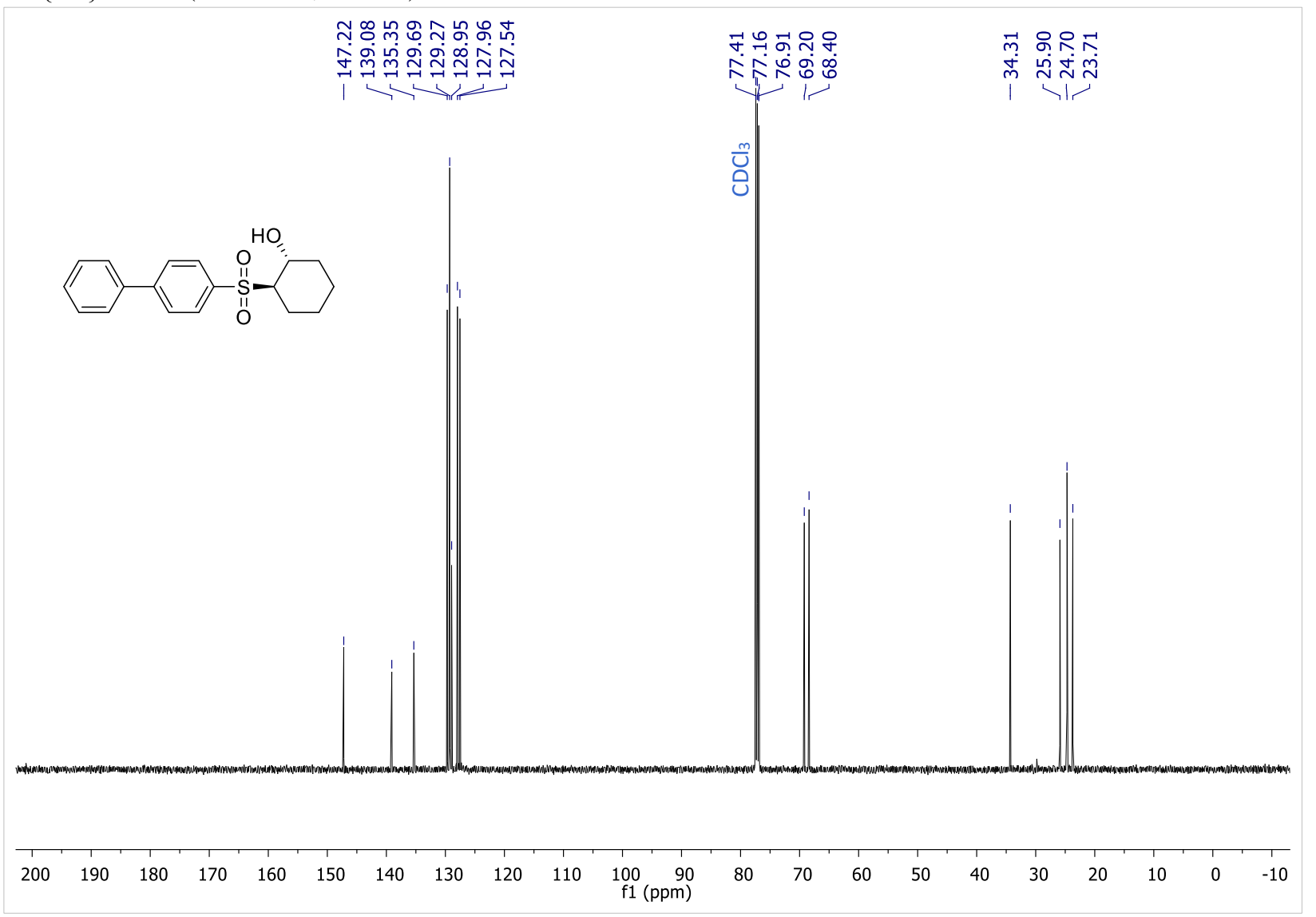


${ }^{1} \mathrm{H} \mathrm{NMR}\left(500 \mathrm{MHz}, \mathrm{CDCl}_{3}\right)$

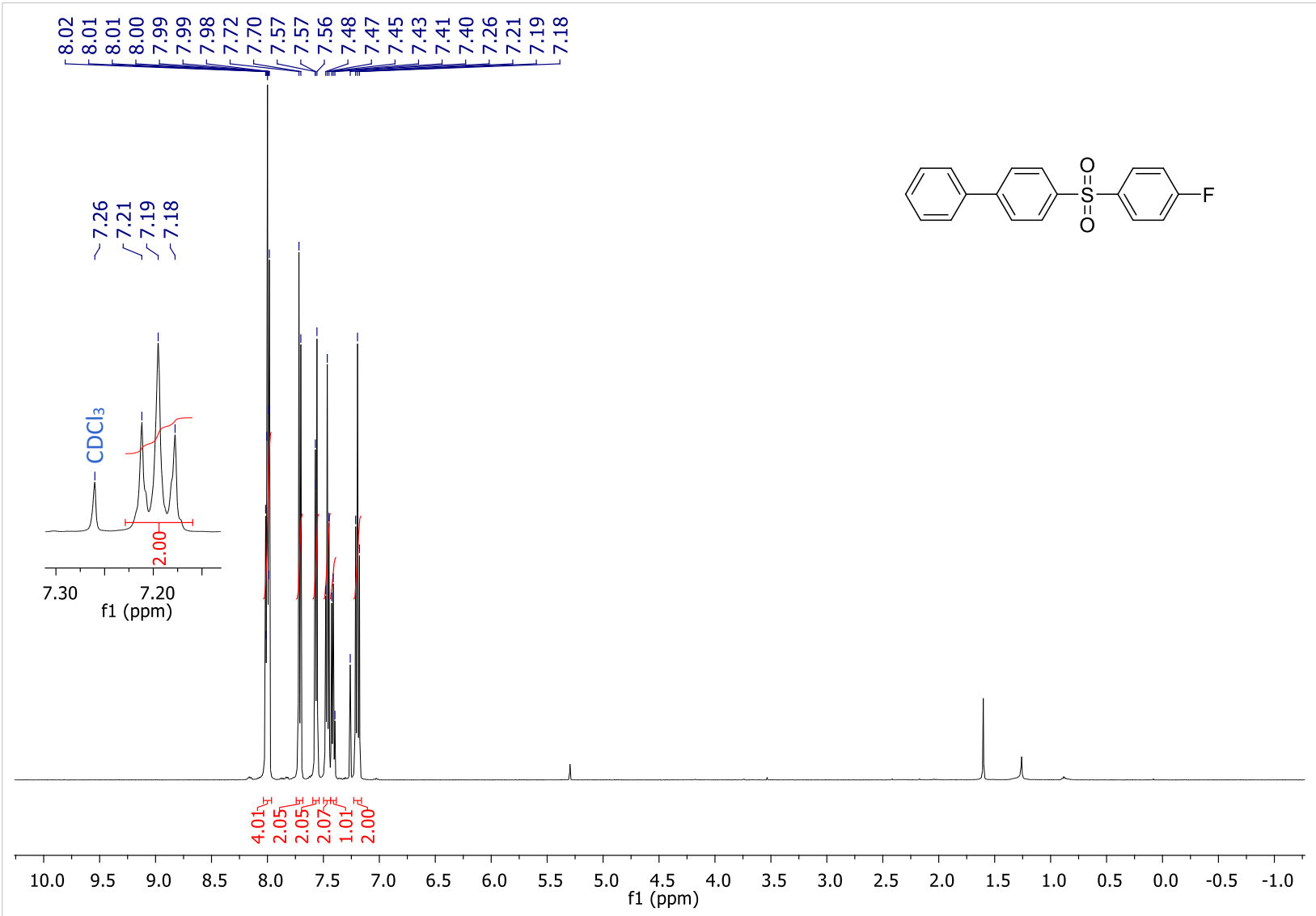

${ }^{13} \mathrm{C}\left\{{ }^{1} \mathrm{H}\right\}$ NMR $\left(126 \mathrm{MHz}, \mathrm{CDCl}_{3}\right)$

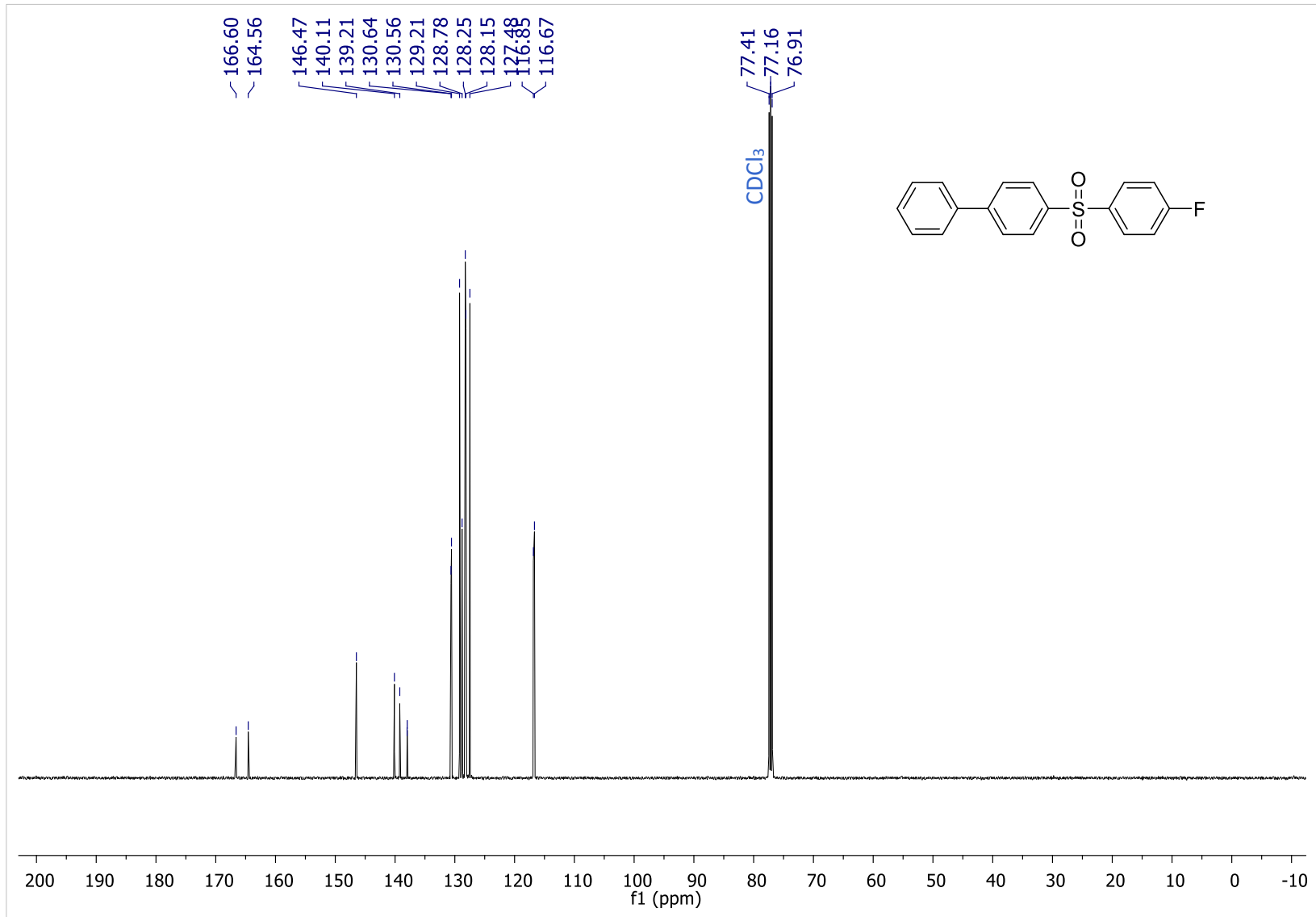


${ }^{1} \mathrm{H}$ NMR $\left(500 \mathrm{MHz}, \mathrm{CDCl}_{3}\right)$

3am

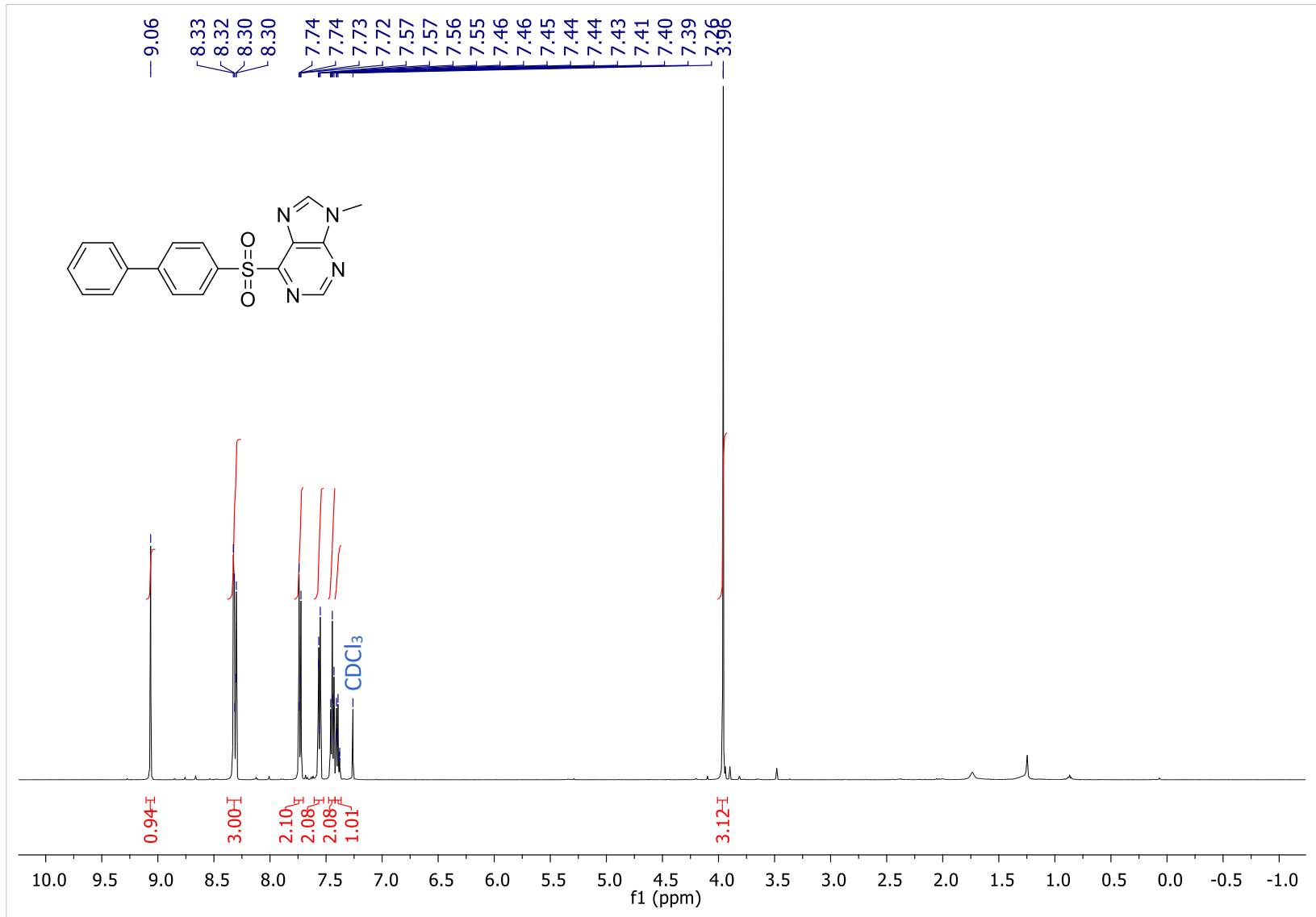

${ }^{13} \mathrm{C}\left\{{ }^{1} \mathrm{H}\right\}$ NMR (126 MHz, $\left.\mathrm{CDCl}_{3}\right)$

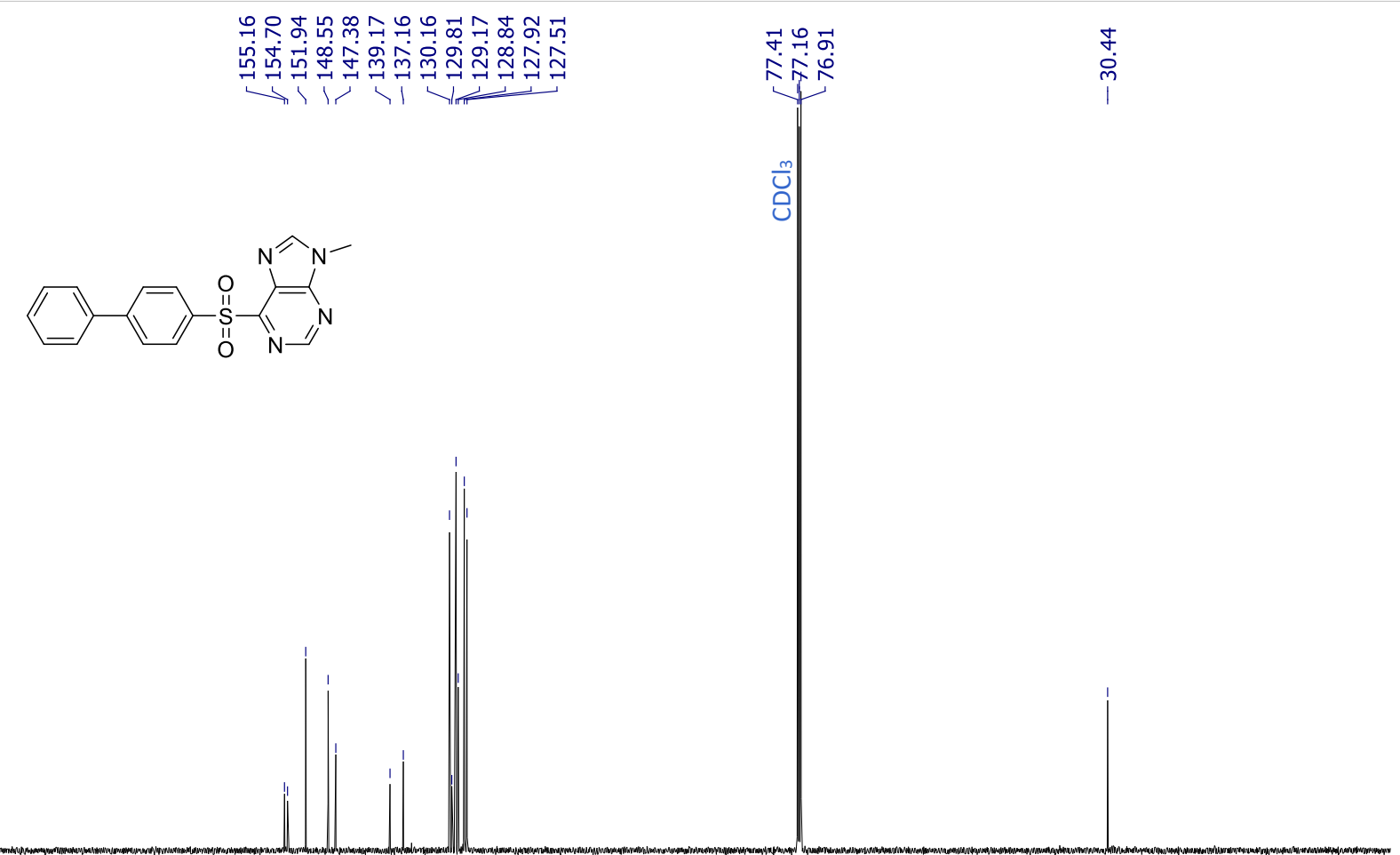




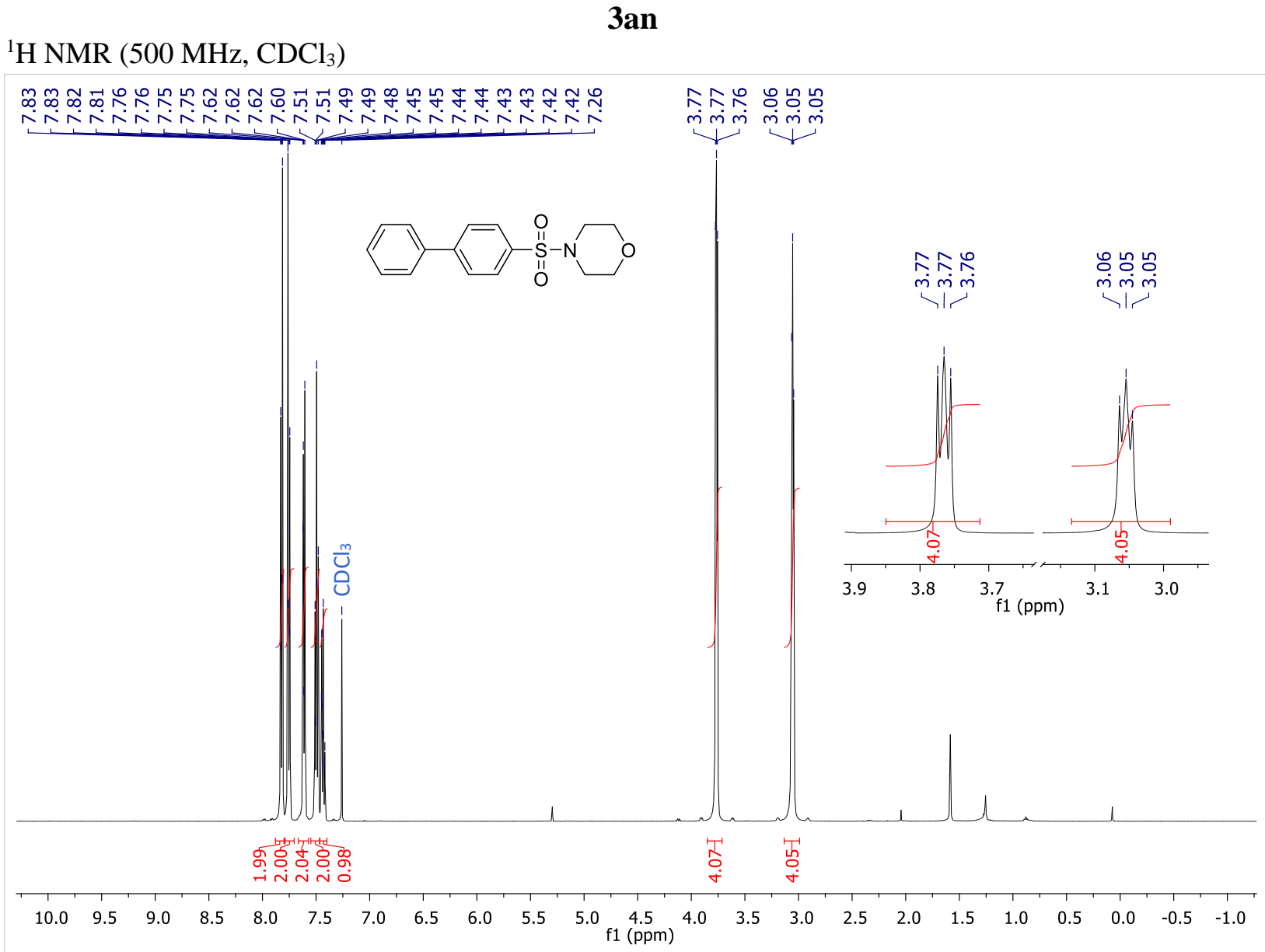

${ }^{13} \mathrm{C}\left\{{ }^{1} \mathrm{H}\right\}$ NMR $\left(126 \mathrm{MHz}, \mathrm{CDCl}_{3}\right)$

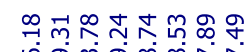

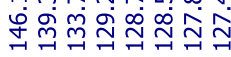

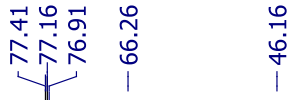

(1)

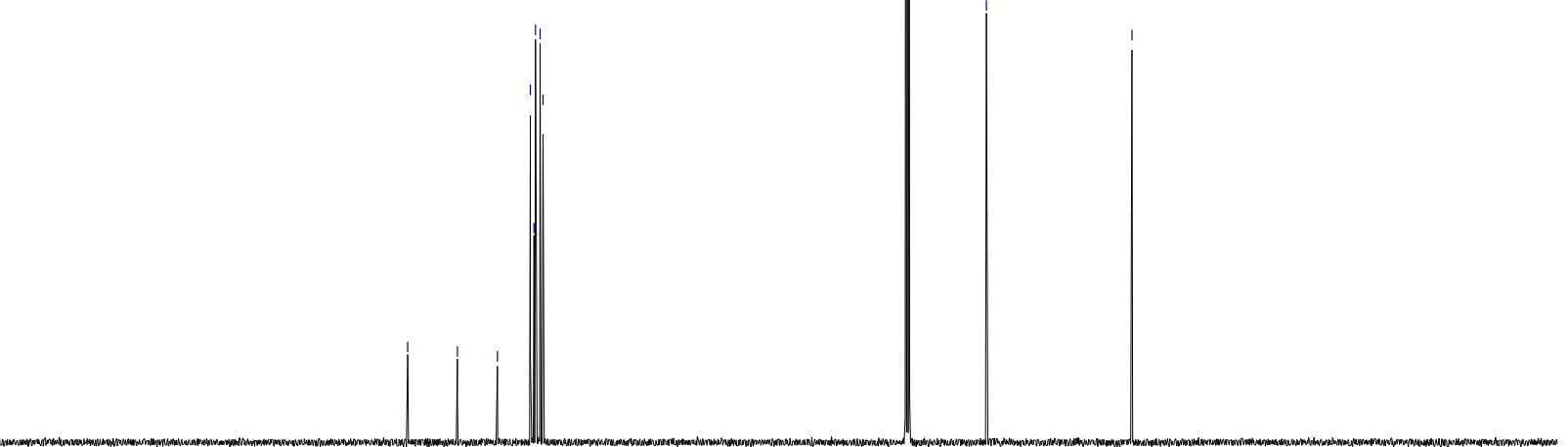

$\begin{array}{llllllllllll}200 & 190 & 180 & 170 & 160 & 150 & 140 & 130 & 120 & 110 & 100 & 90\end{array}$ 


\section{Phenethyl 4-methylbenzenesulfonate}

${ }^{1} \mathrm{H} \mathrm{NMR}\left(500 \mathrm{MHz}, \mathrm{CDCl}_{3}\right)$

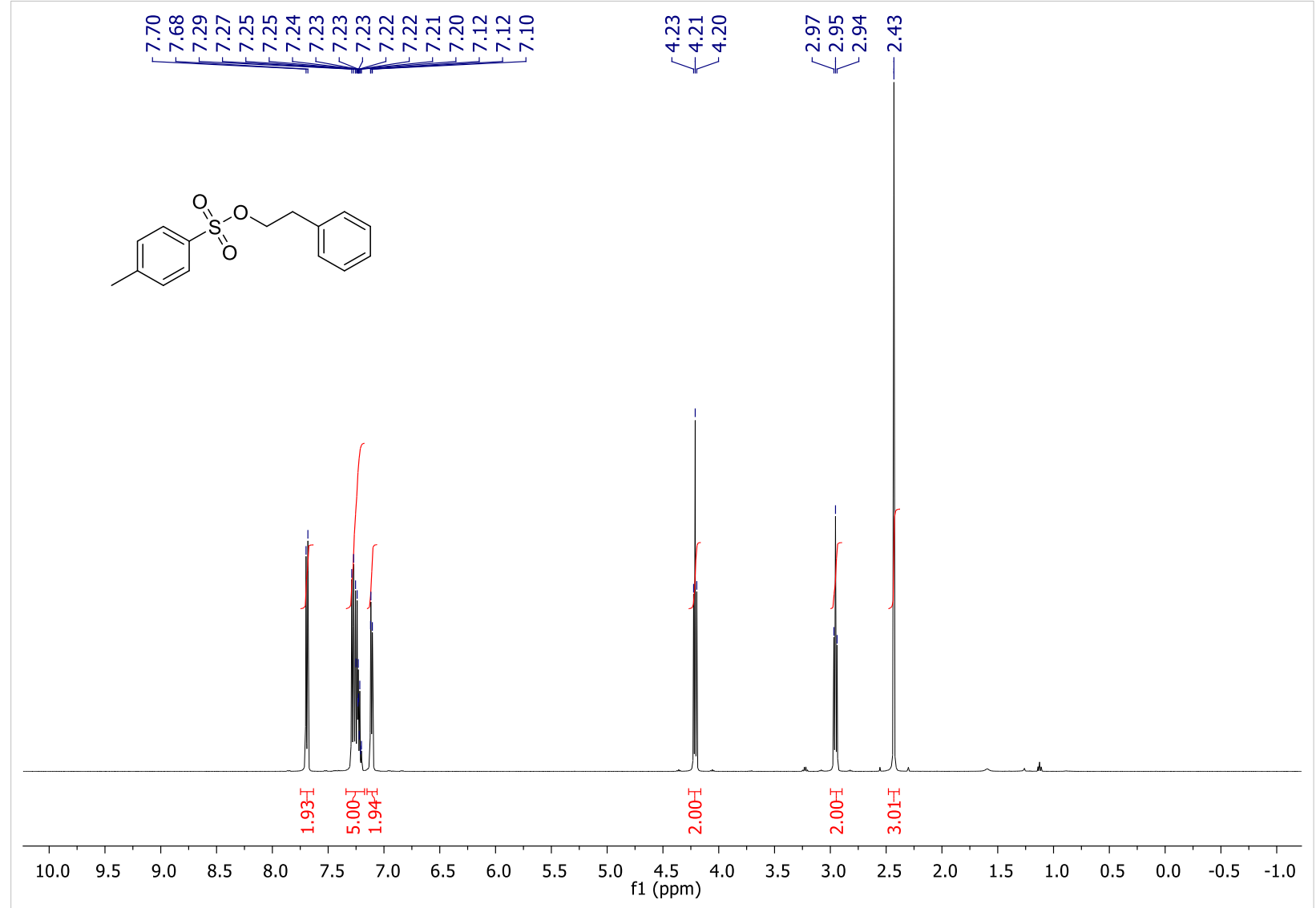

${ }^{13} \mathrm{C}\left\{{ }^{1} \mathrm{H}\right\}$ NMR (126 MHz, $\left.\mathrm{CDCl}_{3}\right)$

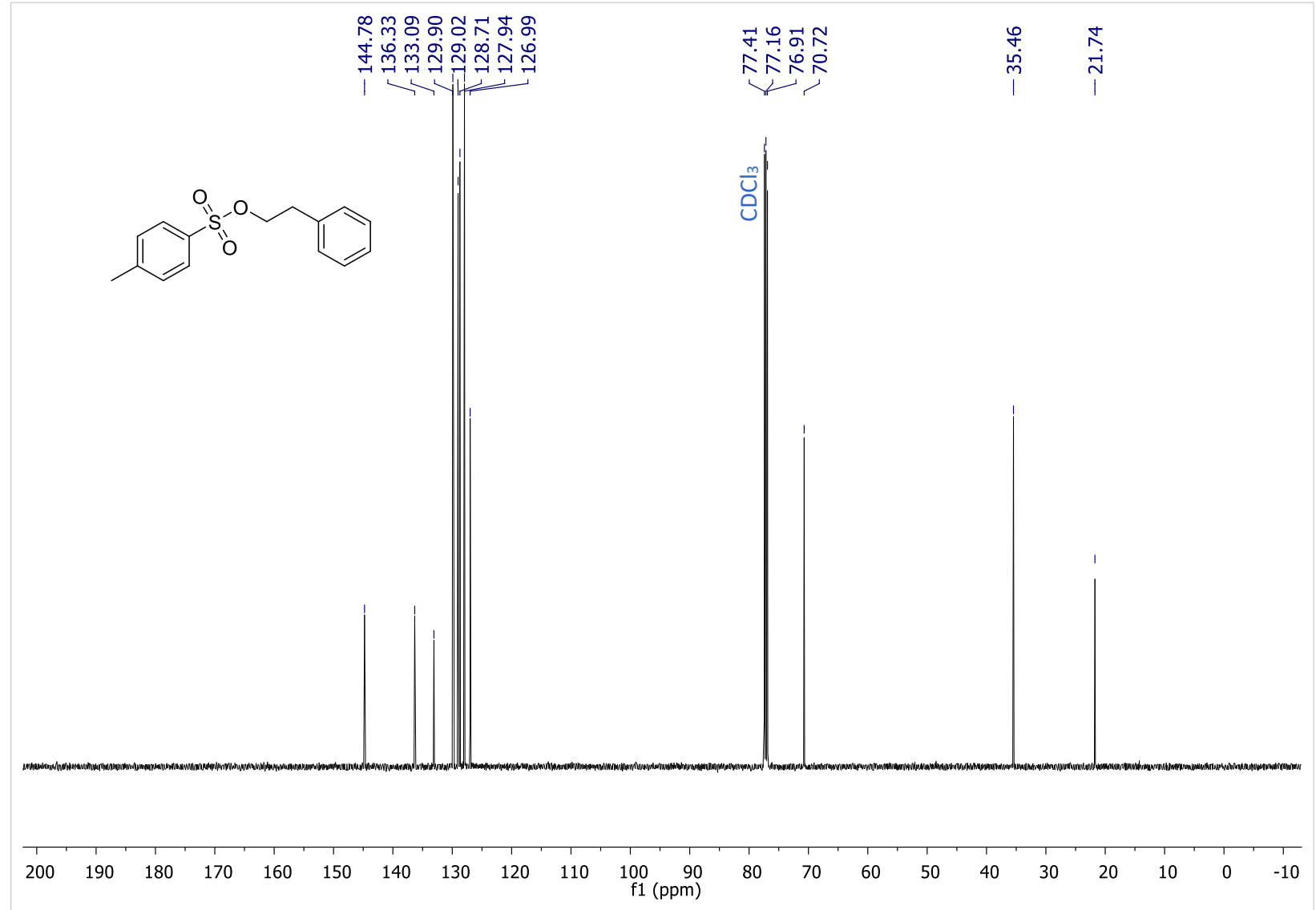




\section{Bis(4-fluorophenyl)iodonium triflate}

${ }^{1} \mathrm{H}$ NMR (500 MHz, MeOD- $\left.d_{4}\right)$

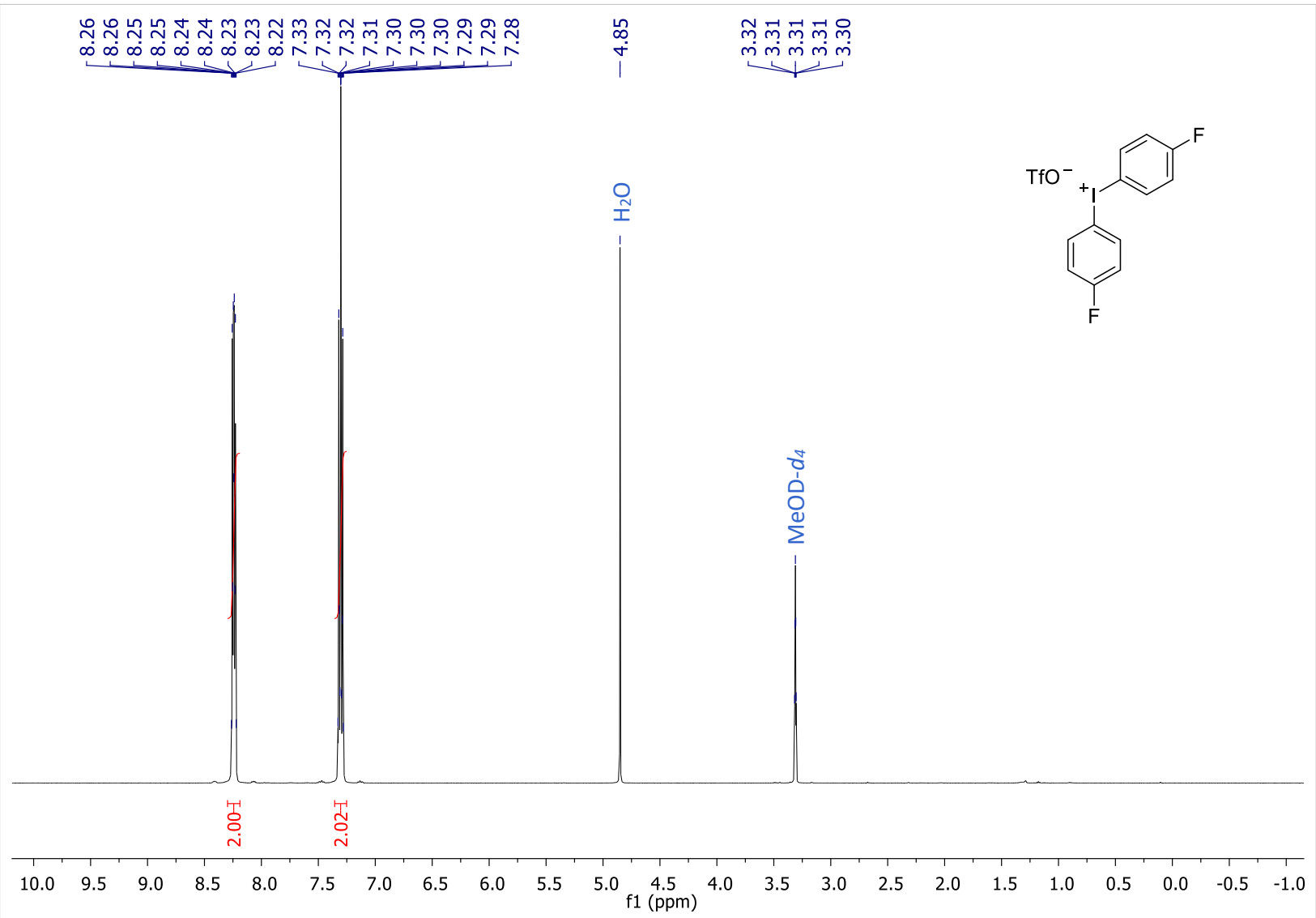

${ }^{13} \mathrm{C}\left\{{ }^{1} \mathrm{H}\right\}$ NMR (126 MHz, MeOD- $\left.d_{4}\right)$

夺.
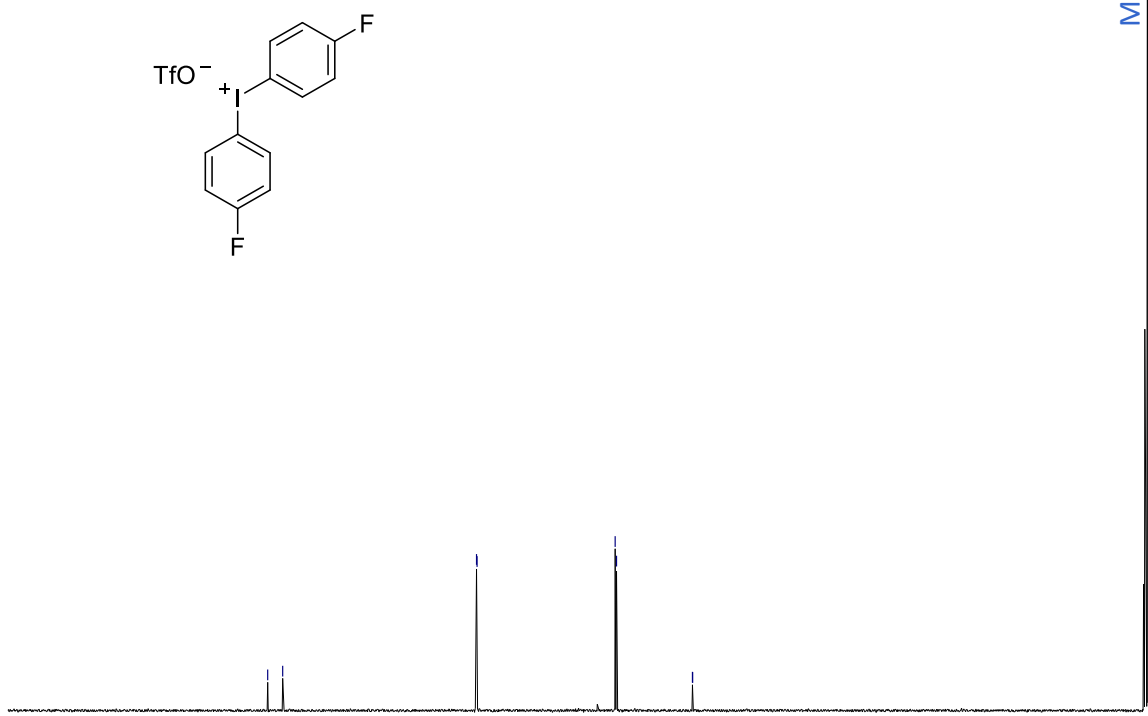


\section{6-Chloro-9-methyl-9H-purine}

${ }^{1} \mathrm{H}$ NMR (500 MHz, DMSO- $\left.d_{6}\right)$

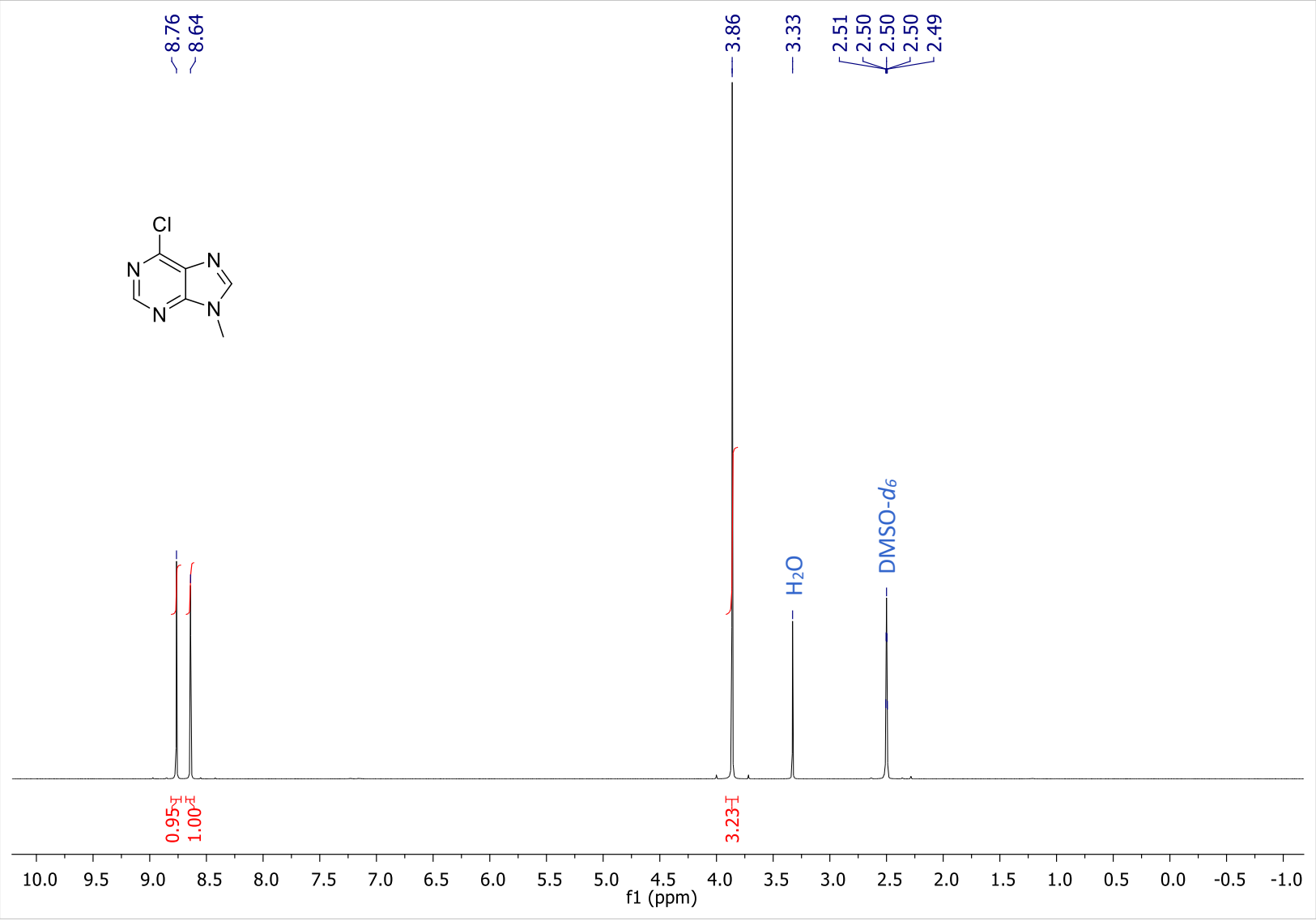

${ }^{13} \mathrm{C}\left\{{ }^{1} \mathrm{H}\right\}$ NMR (126 MHz, DMSO- $\left.d_{6}\right)$

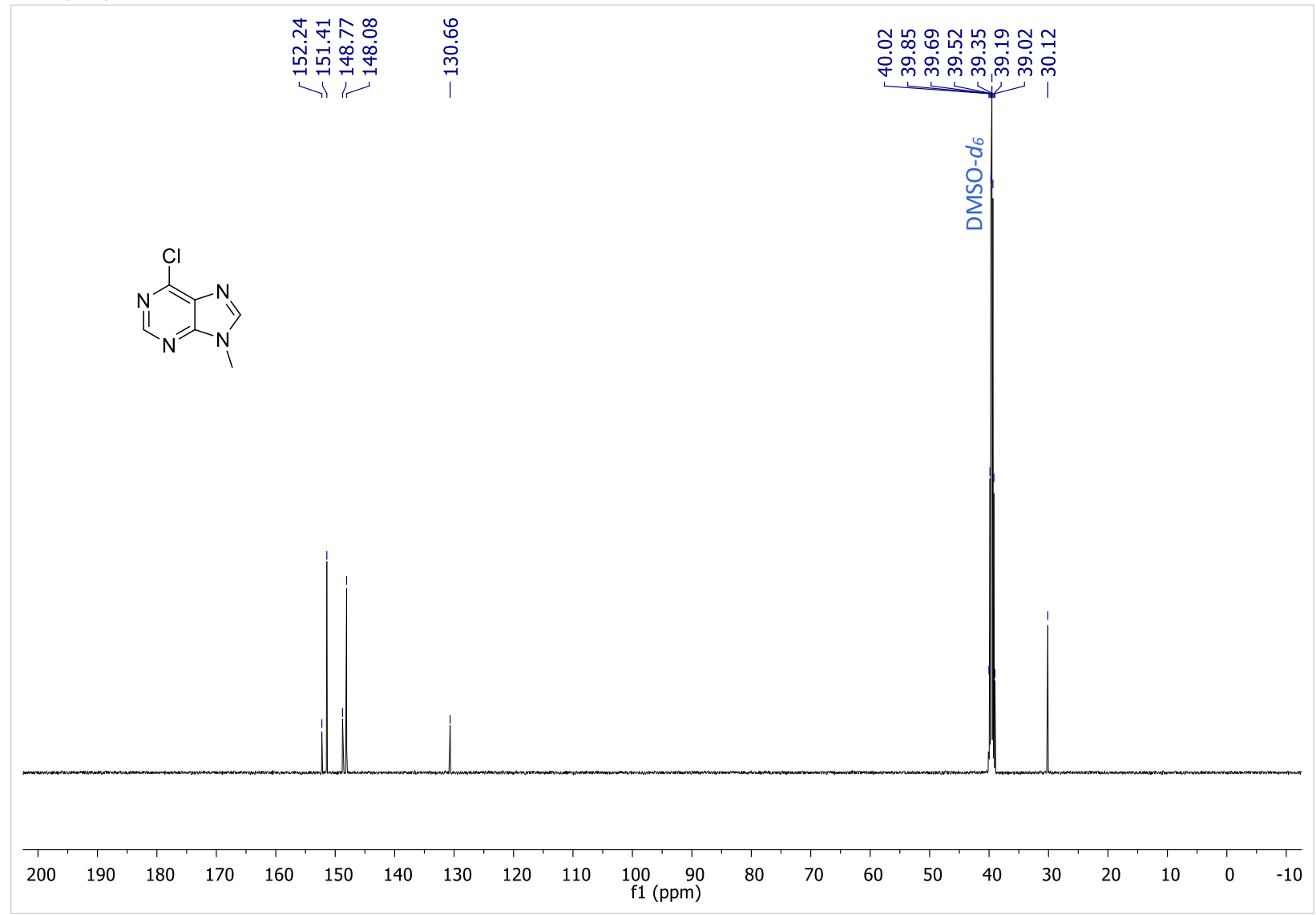

